ES/ER/TM-16

DE93 005175

\title{
Requirements for Quality Control of Analytical Data for the Environmental Restoration Program
}

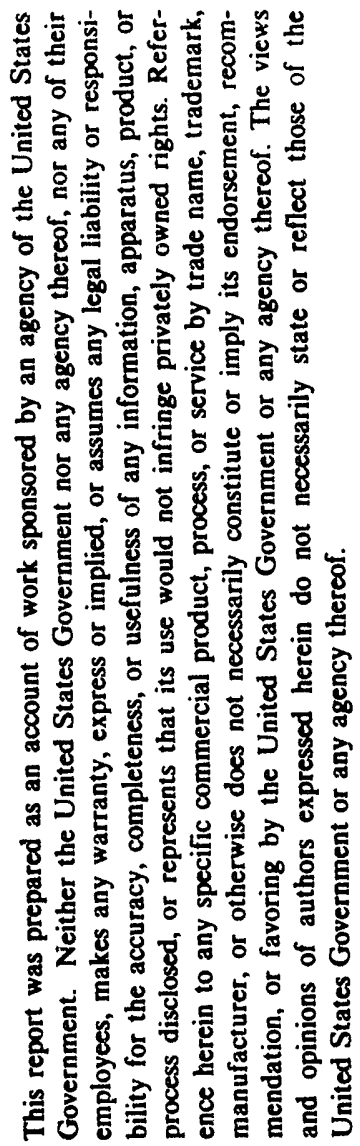

\author{
Environmental Restoration Program \\ P.O. Box 2003 \\ Oak Ridge, Tennessee 37831-7298
}

Date Issued-December 1992

\author{
Prepared by \\ Sampling and Environmental Support Department \\ Oak Ridge K-25 Site \\ Prepared for \\ U.S. Department of Energy
}

Office of Environmental Restoration and Waste Management

under budget and reporting code EW 20

MARTIN MARIETTA ENERGY SYSTEMS, INC.

Oak Ridge K-25 Site

Oak Ridge Y-12 Plant

Oak Ridge National Laboratory under contract DE-AC05-840R21400 managing the

Paducah Gaseous Diffusion Plant Portsmouth Gaseous Diffusion Plant under contract DE-AC05-760R00001

for the

U.S. DEPARTMENT OF ENERGY 


\section{Requirements for Quality Control of Analytical Data for the Environmental Restoration Program}

PREPARED BY:

J. Engels, Projeet Manager

Analytical Environmental Support Group

Sampling and Environmental Support

Department

Analytical Services Division

Martin Marietta Energy Systems, Inc.
CONCURRENCE:

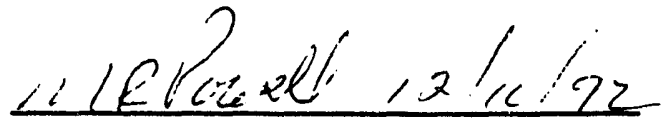

M. Powell, Department Manager

Sampling and Environmental Support

Department

Analytical Services Division

Martin Marietta Energy Systems, Inc.

APPROVED BY:

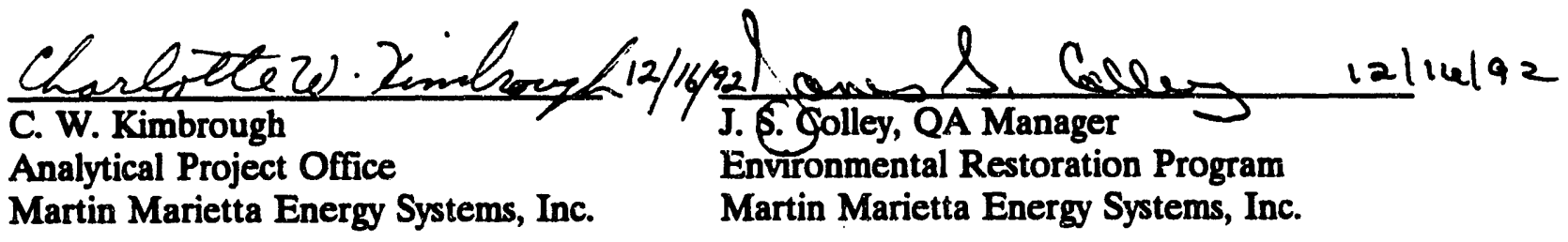
Martin Marietta Energy Systems, Inc. Environmental Restoration Program Martin Marietta Energy Systems, Inc.

Havid m. anden 12/16/92

D. M. Carden, Program Manager DOE-OR, Environmental Restoration 


\section{CONTENTS}

FIGURES $\ldots \ldots \ldots \ldots \ldots \ldots \ldots \ldots \ldots \ldots \ldots \ldots \ldots \ldots \ldots \ldots$

TABLES $\ldots \ldots \ldots \ldots \ldots \ldots \ldots \ldots \ldots \ldots \ldots \ldots \ldots \ldots \ldots \ldots \ldots \ldots$

ACRONYMS $\ldots \ldots \ldots \ldots \ldots \ldots \ldots \ldots \ldots \ldots \ldots \ldots \ldots \ldots \ldots \ldots \ldots \ldots \ldots$

1. INTRODUCTION AND PURPOSE $\ldots \ldots \ldots \ldots \ldots \ldots \ldots \ldots \ldots \ldots \ldots \ldots \ldots$

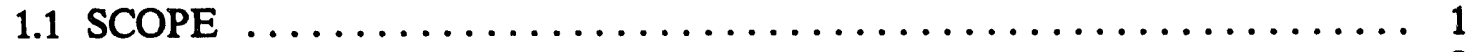

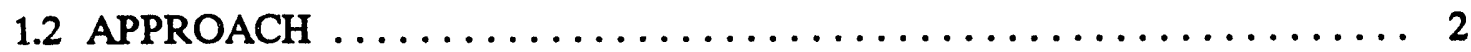

2. ROLES AND RESPONSIBILITIES $\ldots \ldots \ldots \ldots \ldots \ldots \ldots \ldots \ldots \ldots \ldots$

2.1 DOE OAK RIDGE FIELD OFFICE $\ldots \ldots \ldots \ldots \ldots \ldots \ldots \ldots \ldots \ldots \ldots$

2.2 CONTRACTOR ER PROGRAM MANAGEMENT ............ 4

2.3 ANALYTICAL PROJECT OFFICE $\ldots \ldots \ldots \ldots \ldots \ldots \ldots \ldots \ldots \ldots \ldots$

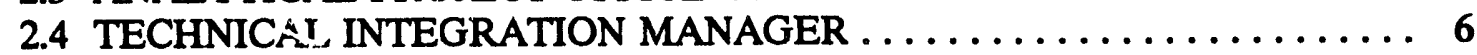

2.5 CONTRACTOR PROJECT MANAGER ............... 6

2.6 MANAGEMENT OF ASSOCIATED LONG-TERM PROGRAMS $\ldots \ldots \ldots 7$

2.7 ER QUALITY ASSURANCE MANAGER AND SPECIALISTS . . . . . . 7

2.8 ER TECHNICAL SUPPORT $\ldots \ldots \ldots \ldots \ldots \ldots \ldots \ldots \ldots \ldots \ldots \ldots$

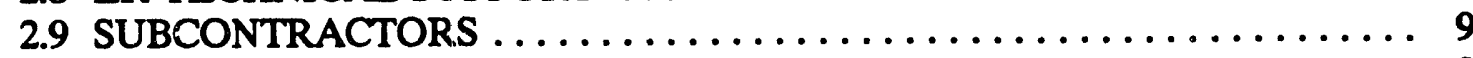

2.10 ANALYTICAL LABORATORY $\ldots \ldots \ldots \ldots \ldots \ldots \ldots \ldots \ldots \ldots$

3. DATA QUALITY OBJECTIVES AND LEVELS

OF QUALTTY CONTROL $\ldots \ldots \ldots \ldots \ldots \ldots \ldots \ldots \ldots \ldots \ldots \ldots \ldots \ldots \ldots$

3.1 DATA QUALITY OBJECTIVES $\ldots \ldots \ldots \ldots \ldots \ldots \ldots \ldots \ldots \ldots \ldots \ldots \ldots \ldots$

3.2 LEVELS A AND B QUALITY CONTROL . . . . . . . . . . . . 14

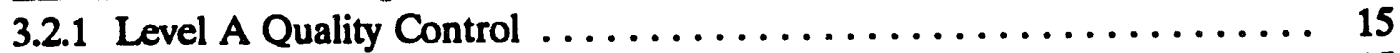

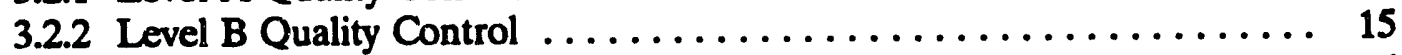

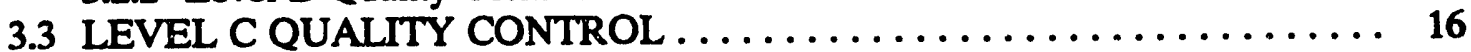

3.4 LEVEL D QUALITY CONTP.OL $\ldots \ldots \ldots \ldots \ldots \ldots \ldots \ldots \ldots \ldots \ldots \ldots \ldots$

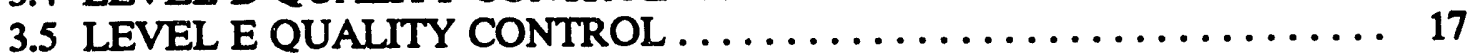

4. PROJECT REQUIREMENTS ....................... 19

4.1 IDENTIFICATION OF DATA QUALITY OBJECTIVES $\ldots \ldots \ldots \ldots \ldots \ldots$

4.2 DEVELOPMENT AND APPROVAL OF THE PROJECT WORK PLAN . 20

4.2.1 Suggested Content of Work Plans .................. 21

4.2.2 Suggested Content of Sampling and Analysis Plans . . . . . . . . . 22

4.3 DATA REVIEW REQUIREMENTS $\ldots \ldots \ldots \ldots \ldots \ldots \ldots \ldots \ldots \ldots \ldots \ldots$

4.4 PROJECT FINAL REPORT ...................... 26

5. SPECIFIC ENVIRONMENTAL RESTORATION REQUIREMENTS . . . . . 28 5.1 LABORATORY REVIEW PROGRAM REQUIREMENTS . . . . . . . . 28

5.1.1 Laboratory Quality Assurance Plan Requirements . . . . . . . . . . . . 29

5.1.2 Performance Evaluation Sample Requirements ............. 32 
5.1.3 Initial Laboratory Audit Requirements . . . . . . . . . . . . . . 32

5.1 .4 Continuing Laboratory Reviews $\ldots \ldots \ldots \ldots \ldots \ldots \ldots \ldots \ldots \ldots$

5.2 OPERATIONAL REQUIREMENTS $\ldots \ldots \ldots \ldots \ldots \ldots \ldots \ldots \ldots \ldots \ldots$

5.2.1 Laboratory Organization and Personnel $\ldots \ldots \ldots \ldots \ldots \ldots \ldots \ldots$

5.2.2 Standard Operating Procedures . . . . . . . . . . . . . . . 37

5.2 .3 Holding Time Requirements $\ldots \ldots \ldots \ldots \ldots \ldots \ldots \ldots \ldots \ldots \ldots \ldots$

5.2 .4 Sample Receipt Requirements $\ldots \ldots \ldots \ldots \ldots \ldots \ldots \ldots \ldots \ldots \ldots . \ldots \ldots$

5.2 .5 Chain-of-Custody Requirements ................. 38

5.2 .6 Error Correction Requirements . . . . . . . . . . . . . . . 39

5.2.7 Requirements for Cleanliness of Sample Containers ........... 39

5.2.8 Requirements for Reporting Out-of-Control Events ........... 40

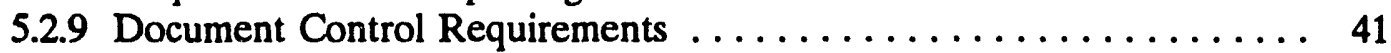

5.2.10 Requirements for Traceability of Standards ............. 41

5.3 LABORATORY QUALITY CONTROL SAMPLES $\ldots \ldots \ldots \ldots \ldots \ldots \ldots 41$

5.3 .1 Method Blank ........................ 41

5.3.2 Laboratory Control Samples ..................... 42

5.3.3 Matrix Spikes and Matrix Spike Duplicates .............. 42

5.3.4 Duplicate Samples .......................... 43

5.4 LABORATORY CONTROL SAMPLE PROGRAM REQUIREMENTS ... 43

5.4.1 Control Charts ....................... 44

5.4.2 Administration of Control Charts ................. 44

5.4.3 Statistical Approach to Control Charts ................. 44

5.4.4 Minimum Statistical Control Charting ................ 45

5.4.5 Criteria for an Out-of-Control Condition ............... 45

5.4.6 Reactions to Out-of-Control Conditions on Control Samples . . . . . . . 46

5.5 ANALYTICAL METHOD REQUIREMENTS ............... 46

6. REQUIREMENTS FOR SAMPLE ANALYSIS

DELIVERABLES $\ldots \ldots \ldots \ldots \ldots \ldots \ldots \ldots \ldots \ldots \ldots \ldots \ldots \ldots \ldots$

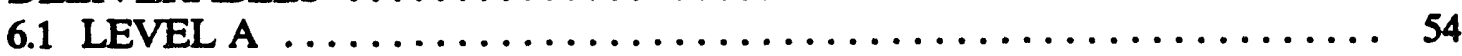

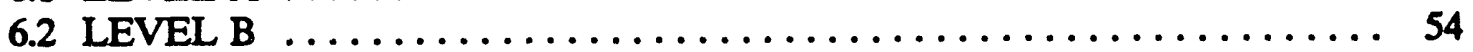

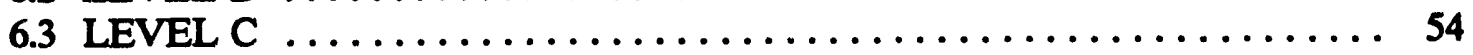

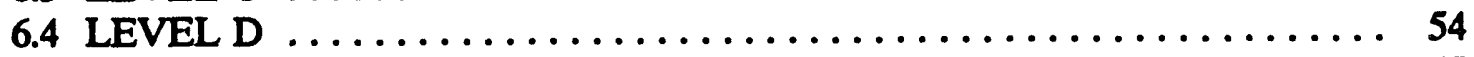

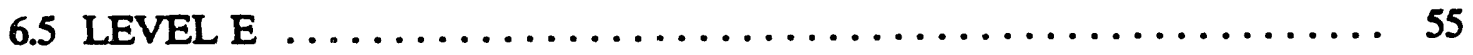

7. DATA VALIDATION GUIDELINES $\ldots \ldots \ldots \ldots \ldots \ldots \ldots \ldots \ldots \ldots$

7.1 LEVEL A DATA VALIDATION GUIDELINES ............. 59

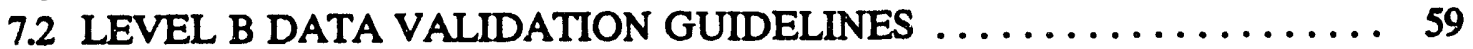

7.3 LEVEL C DATA VALIDATION GUIDELINES ............. 60

7.3.1 Petroleum Hydrocarbons (EPA Method 418.1) ............ 68

7.3.2 Gas Chromatograph/Mass Spectrometer Volatile Organics ......... 70

7.3.3 Gas Chromatograph Volatile Organics ................. 75

7.3.4 Gas Chromatography/Mass Spectrometer Semivolatile Organics ...... 78

7.3.5 Pesticides/Polychlorinated Biphenyls ................. 84

7.3.6 Metals and Cyanide $\ldots \ldots \ldots \ldots \ldots \ldots \ldots \ldots \ldots \ldots \ldots \ldots . \ldots \ldots$

7.3.7 Wet Chemistry ................................. 100

7.3.8 Radiochemical Analysis ........................ 101

7.4 LEVEL D DATA VALIDATION GUIDELINES . . . . . . . . . . . . . . 104 
7.5 LEVEL E DATA VALIDATION GUIDELINES . . . . . . . . . . 104

7.6 DEFINITION OF DATA QUALIFIERS $\ldots \ldots \ldots \ldots \ldots \ldots \ldots \ldots \ldots . \ldots \ldots$

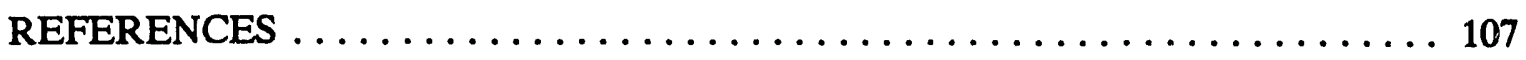

BIBLIOGRAPHY $\ldots \ldots \ldots \ldots \ldots \ldots \ldots \ldots \ldots \ldots \ldots \ldots \ldots \ldots \ldots \ldots$

GLOSSARY $\ldots \ldots \ldots \ldots \ldots \ldots \ldots \ldots \ldots \ldots \ldots \ldots \ldots \ldots \ldots \ldots \ldots \ldots \ldots \ldots \ldots$

v 


\section{FIGURES}

1.1 Flowdown of Environmental Restoration quality assurance requirements ... 3

2.1 Environmental Restoration team functional organization $\ldots \ldots \ldots \ldots \ldots$

\section{TABLES}

3.1 Data quality objective and quality control levels $\ldots \ldots \ldots \ldots \ldots \ldots \ldots$

3.2 Quality control level and deliverables requirements $\ldots \ldots \ldots \ldots \ldots \ldots$

4.1 Sample portion of data summary tables .................. 24

5.1 Laboratory quality control sample frequency ............... 42

$5.2 \quad$ Analytical methods $\ldots \ldots \ldots \ldots \ldots \ldots \ldots \ldots \ldots \ldots \ldots \ldots \ldots \ldots$

6.1 Data set deliverables for Level $\mathrm{C}$ quality assurance $\ldots \ldots \ldots \ldots \ldots \ldots$

7.1 Environmental Restoration requirements summary for Contract Laboratory Program methods ................. 61

7.2 Environmental Restoration volatile organic requirements .......... 62

7.3 Environmental Restoration extractable organic requirements ........ 63

7.4 Environmental Restoration requirements for other organics .......... 64

7.5 Environmental Restoration metals requirements ............. 65

7.6 Environmental Restoration radiochemical requirements .......... 66

7.7 Environmental Restoration requirements for general chemical analyses ... 67

7.8 Minimum relative response factors for initial calibration of volatile organics-CLP SOW Revision OLM01.8 ................ 71

7.9 Compounds that must meet a minimum RRF criterion of $0.010 \ldots \ldots \ldots$

7.10 Minimum response factors for initial calibration of semivolatile organies-CLP SOW Revision OLM01.8 ................ 80

7.11 Compounds that must meet a minimum RRF criterion of $0.010 \ldots \ldots \ldots$

7.12 Analyte concentration equivalent (milligram per liter) arising from interferants at $100 \mathrm{mg} / \mathrm{L} \ldots \ldots \ldots \ldots \ldots \ldots \ldots \ldots \ldots$ 


\section{ACRONYMS}

\begin{tabular}{|c|c|}
\hline AA & atomic absorption \\
\hline $\mathrm{ACD}$ & Analytical Chemistry Department \\
\hline ADMI & American Dye Manufacturer's Institute \\
\hline AESG & Analytical Environmental Support Gruup \\
\hline APO & Analytical Project Office \\
\hline ARARs & Applicable or Relevant and Appropriate Requirements \\
\hline ASTM & American Society for Testing and Materials \\
\hline $\mathrm{CCC}$ & calibration check compounds \\
\hline $\mathrm{CCV}$ & calibration check verification \\
\hline CERCLA & Comprehensive Environmental Response, Compensation, and Liability Act \\
\hline CFR & Code of Federal Regulations \\
\hline CLP & Contract Laboratory Program \\
\hline $\mathrm{COC}$ & chain of custody \\
\hline CRDL & contract-required detection limit \\
\hline CRP & Community Relations Plan \\
\hline CRQL & contract-required quantitation limits \\
\hline CVAA & cold-vapor atomic absorption \\
\hline$\% \mathrm{D}$ & percent difference \\
\hline $\mathrm{DBC}$ & dibutylchlorendate \\
\hline DCRC & Document Content and Response Committee \\
\hline DDD & dichlorodiphenyldichloroethane \\
\hline DDE & dichlorodiphenylethane \\
\hline DDT & dichlorodiphenyltrichloroethane \\
\hline DOE & U.S. Department of Energy \\
\hline DOE-OR & DOE Oak Ridge Field Office \\
\hline DQO & data quality objective \\
\hline EDT & electronic data transmission \\
\hline EDTA & ethylenediaminetetraacetic acid \\
\hline EPA & U. S. Environmental Protection Agency \\
\hline ER & Environmental Restoration \\
\hline FAA & furnace atomic absorption \\
\hline FSP & Field Sampling Plan \\
\hline GC & gas chromatograph \\
\hline GCMS & gas chromatograph/mass spectrometer \\
\hline GFAA & graphite furnace atomic absorption \\
\hline GPC & gel permeation chromatography \\
\hline HASP & Health and Safety Plan \\
\hline HPLC & high-performance liquid chromatography \\
\hline ICP & inductively coupled plasma \\
\hline ICS & interference check sample \\
\hline IDL & instrument detection limit \\
\hline IS & internal standard \\
\hline ISO & International Standards Organization \\
\hline LCS & laboratory control sample \\
\hline
\end{tabular}




$\begin{array}{ll}\text { LQAC } & \text { Laboratory Quality Assurance Coordinator } \\ \text { LQAP } & \text { Laboratory Quality Assurance Plan } \\ \text { LUFT } & \text { leaking underground fuel tank } \\ \text { MDA } & \text { minimum detectable activity } \\ \text { MPR } & \text { Monthly Progress Report } \\ \text { MS } & \text { matrix spike } \\ \text { MSA } & \text { Method of Standard Addition } \\ \text { MS/MSD } & \text { matrix spike/matrix spike duplicate } \\ \text { NCP } & \text { National Contingency Plan } \\ \text { NIST } & \text { National Institute of Standards and Testing } \\ \text { NPDES } & \text { National Pollutant Discharge Elimination System } \\ \text { NPL } & \text { National Priorities List } \\ \text { OSWER } & \text { Office of Solid Waste and Emergency Response } \\ \text { PCB } & \text { polychlorinated biphenyl } \\ \text { PE } & \text { Performance Evaluation } \\ \text { PEM } & \text { Performance Evaluation Mixture } \\ \text { QA } & \text { quality assurance } \\ \text { QAPP } & \text { Quality Assurance Project Plan } \\ \text { QC } & \text { quality control } \\ \text { \%R } & \text { percent recovery } \\ \text { RCRA } & \text { Resource Conservation and Recovery Act } \\ \text { RPD } & \text { relative percent difference } \\ \text { RRF } & \text { relative response factor } \\ \text { \%RSD } & \text { percent relative standard deviation } \\ \text { SAP } & \text { Sampling and Analysis Plan } \\ \text { SOP } & \text { standard operating procedure } \\ \text { SOW } & \text { statement of work } \\ \text { SPARCC } & \text { Sensitivity, Precision, Accuracy, Representativeness, Comparability, and } \\ \text { TCL } & \text { Completeness } \\ \text { TCLP } & \text { target compound list } \\ \text { TIC } & \text { Toxicity Characteristic Leaching Procedure } \\ \text { TPH } & \text { tentatively identified (organic) compounds } \\ \text { VOC } & \text { total petroleum hydrocarbons } \\ \text { wM } & \text { volatile organic compound } \\ \text { WP } & \text { Waste Management } \\ & \text { Work Plan } \\ & \end{array}$




\section{INTRODUCTION AND PURPOSE}

The Environmental Restoration (ER) Program was established for the investigation and remediation of inactive U.S. Department of Energy (DOE) sites and facilities that have been declared surplus in terms of their previous uses. Such sites are treated according to the National Contingency Plan (NCP) of the Comprehensive Environmental Response, Compensation, and Liability Act (CERCLA). The NCP provides procedures for the identification, evaluation, and remediation of past hazardous waste disposal sites. The Hazardous Materials Response section of the NCP consists of several phases: Preliminary Assessment, Site Inspection, Remedial Investigation, Feasibility Study, Remedial Design, and Remedial Action. During any of these phases, analysis of soil, water, and waste samples may be performed.

The DOE Oak Ridge Field Office (DOE-OR) administers ER activities. The Martin Marietta Energy Systems, Inc., ER Division is involved in performing pursuant field investigations and sample analyses for Oak Ridge and for integrating the efforts of all ER participants, as discussed in Sect. 2.

The purpose of this document is to specify ER requirements for quality control (QC) of analytical data. Activities throughout all phases of the investigation may affect the quality of the final data product, thus are subject to control specifications. Laboratory control is emphasized in this document, and field concerns will be addressed in a companion document. Energy Systems, in its role of technical coordinator and at the request of DOE-OR, extends the application of these requirements to all participants in ER activities. Because every instance and concern may not be addressed in this document, participants are encouraged to discuss any questions with the ER Quality Assurance (QA) Office, the Analytical Environmental Support Group (AESG), or the Analytical Project Office (APO).

\subsection{SCOPE}

The provisions of this document apply to all ER projects under DOE-OR jurisdiction, including activities of contractors, subcontractors, and selected analytical laboratories conducting remedial response actions.

Laboratories performing studies in support of ER projects are required to pass ER review before beginning field studies or analyses of samples and to maintain active status throughout duration of the studies. This document provides the requirements that laboratories must follow to pass review and maintain active status. Should more than one laboratory be involved in the analysis of samples from a single site, each laboratory performing analysis must undergo review and must comply with the QC requirements specified in this document. These objectives and requirements conform, in general, with the following:

- "Toxic Substances Control; Good Laboratory Practice Standards; Final Rule," U.S. Environmental Protection Agency, Federal Register, Vol. 48, November 29, 1983. 
- "Nonclinical Laboratory Studies; Good Laboratory Practice Regulations," Food and Drug Administration, Federal Register, Vol. 43, December 22, 1978.

- DOE Order 5700.6C (encompasses NQA-1), American National Standards Institute/ American Society of Quality Control (ANSI/ASQC) standards (ANSI 1991), and International Standard Organization (ISO) for Standardization 9000, Quality Management and Quality Assurance Standards-Guidelines for Selection and Use (ISO 1987).

Individual projects shall also comply with the Interim Guidelines and Specifications for Preparing Quality Assurance Project Plans, EPA-600/4-83-004, QAMS-005/80, February (EPA 1983) and Environmental Restoration Quality Program Plan, ES/ER/TM-4/R2, September (Energy Systems 1992) and be consistent with Requirements for Quality Control of Field Activities, ES/ER/TM-11 (Energy Systems to be issued in 1993) (see Fig. 1.1).

Each laboratory is required to submit a Labcratory Quality Assurance Plan (LQAP) prior to conducting work for ER projects. The LQAP is emphasized because review of, and adherence to, its contents are essential for obtaining and maintaining ER active status. Certain basic requirements stressed are a laboratory quality assurance coordinator (LQAC); the use of accepted analytical methods; careful documentation of chain of custody (COC); a corrective action policy; submission of monthly progress reports (MPRs); and use of control charts. The laboratory review process and subsequent laboratory reporting requirements provide the mechasisisin for verifying that a laboratory is adhering to the LQAP and the requirements of this document.

\subsection{APPROACH}

The approach reflected in this document is one of outlining requirements and allowing the laboratories, principally through their LQAP, to detail their approach to meeting these requirements. For example, with the exception of the Laboratory Control Sample (LCS) program (see Sect. 5.4), the discussion of QC procedures includes a requirement that warning and control limits be set but allows each laboratory to describe its procedures for establishing such limits. The specific organization and presentation of the LCAP are left largely to the discretion of the laboratory, although certain areas must be addressed.

For this approach to work, emphasis will be placed on effective communication among the laboratory, ER Project manager, APO, QA, and engineering functions. All documents need to be concise, well organized, and free of jargon that might hinder constructive review and evaluation. 
DWG. NO. KG-2-3482RM

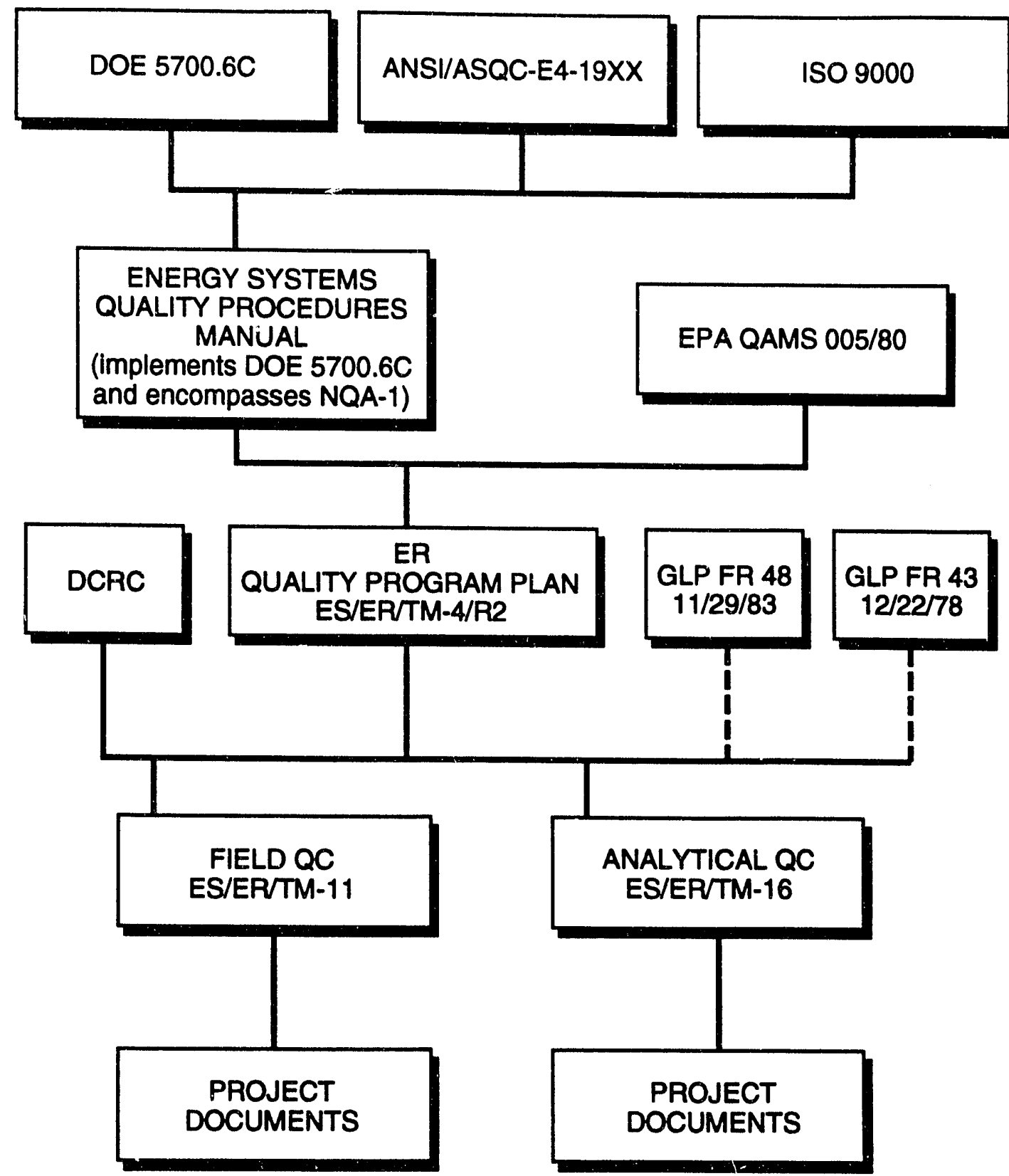

DCRC $=$ Document Content and Response Committee

Fig 1.1. Flowdown of Environmental Restoration quality assurance requirements. 


\section{ROLES AND RESPONSIBILITIES}

As indicated in Fig. 2.1, organizations involved in QC of analytical data are DOE, Energy Systems, their contractors, and subcontractors. Each organization has multiple tasks and groups that support the project. Fig. 2.1 includes the structure of the ER organization relative to environmental programs. A brief description of key roles and responsibilities appears below.

\section{DOE OAK RIDGE FIELD OFFICE}

DOE-OR is responsible for providing ER project funding, direction, and oversight of ER contractors and interfacing with DOE Headquarters and federal and state regulators.

\section{CONTRACTOR ER PROGRAM MANAGEMENT}

Energy Systems ER Program max directing, and controlling all operations in se division. Program management will assist in providing site information, history, and assigning project funding and will specify sites requiring investigation and remediation, provide logistical assistance, review results, and make recommendations. Specific responsibilities include:

- Securing contract laboratories with direction from APO.

- Internal oversight of project QA activities.

- Interfacing between APO and other ER Program managers for technical and QA guidance and requirements.

\section{ANALYTICAL PROJECT OFFICE}

The APO provides aralytical technical support coordination. It serves as the point of contact between external laboratories and other ER participants. Specific responsibilities include:

- Developing statements of work for analytical services.

- Determining appropriate analytical protocols to be applied to meet the data quality objectives (DQO).

- Developing technical and quality standards for requested analytical services.

- Determining the appropriate laboratories with which to place the work.

- Negotiating scheduling of provision of services and oversight to ensure schedule is met.

- Assessing laboratories to ensure compliance with quality and technical standards. 


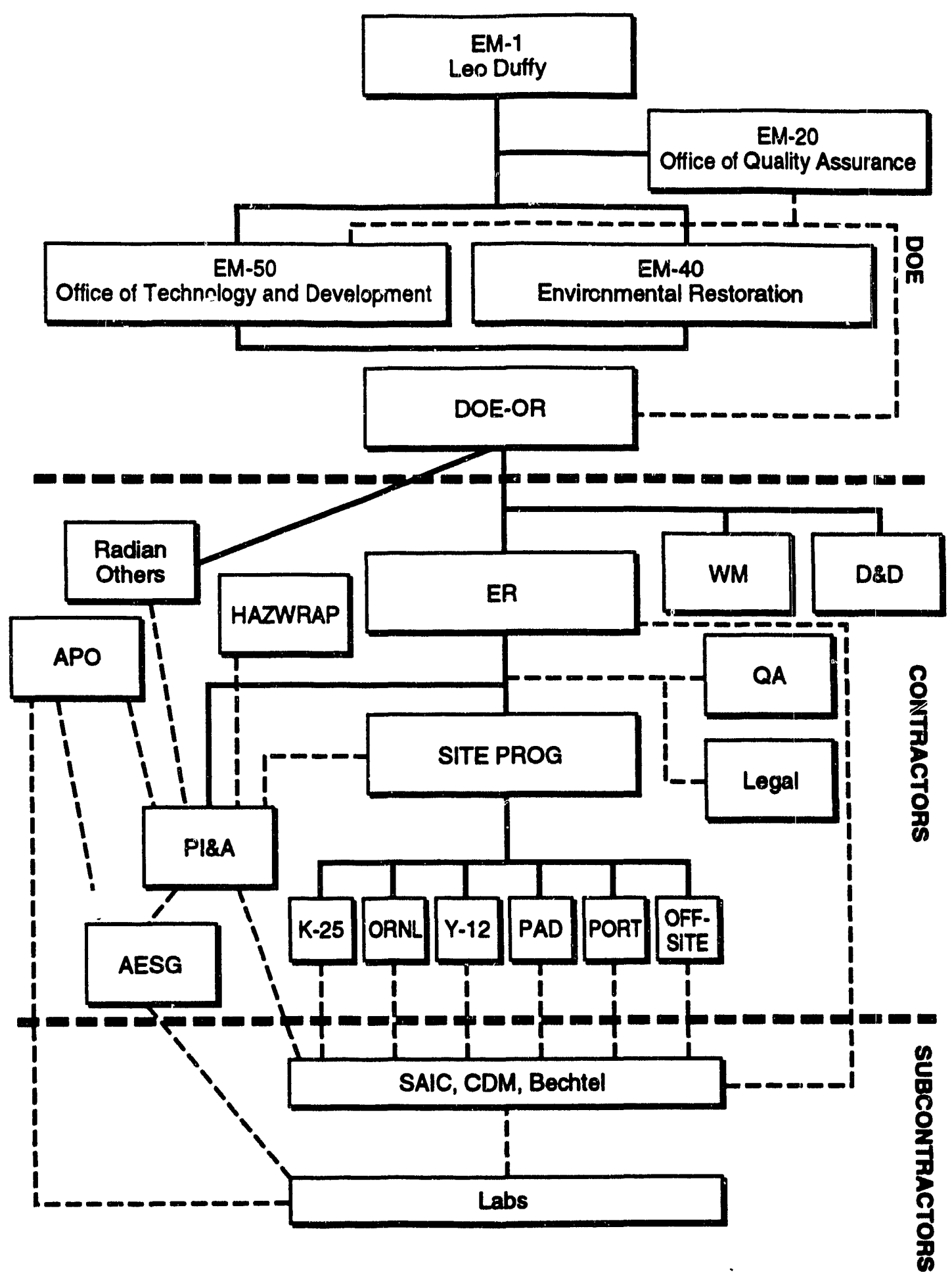

PI\&A = Program Integration and Administration

Fig 21. Environmental Restoration team functional organization. 
- Ensuring appropriate data validation and transmittal to the program customer.

The APO provides or coordinates technical support to several entities at Energy Systems. The primary recipient of this support is ER. APO may matrix other groups in turn to provide services through APO auspices.

The APO is responsible for management of sample o erflow. The meeting point between laboratory and APO sample management responsibilities is still evolving as resourc $\cdots$ and obligations are added. Eventually, parties will rely on arrangements rather than daily cuntacts to direct sample flow.

The APO is responsible for the procurement of subcontracts for direct analytical support for ER and must approve ER nondirect analytical subcontracts and associated task orders (i.e., analytical work performed under general order contracts) and requests $f$ service from plant laboratories. APO approval covers analytical methods, 4 and QC requirements, deliverables, appropriateness of the designated entity to accomplish the work, and any other requirements called out in the analytical plan.

\section{TECHNICAL INTEGRATION MANAGER}

The tecinnical integration manager coordinates issues that deal with multiple sites, projects, or participating entities to maximize efficiency and consistency. Analytical matters are referred to the APO for coordination. The technical integration manager works closely with the APO.

\section{CONTRACTOR PROJECT MANAGER}

The contractor (e.g., Energy Systems) ER project managers are responsible for managing all activities concerned with planning and executing individual projects to meet project cost, schedule, technical, and quality objectives.

Specific responsibilities include:

- Identifying project team members by requesting personnel support from the respective program functions.

- Defining project objectives and subsequent DQOs.

- Planning and directing the collective actions of assigned team members and the ER subcontractors to meet project objectives.

- Identifying project requirements and developing project work plans (WPs) to meet requirements.

- Defining quality requirements for program procurement documents and other program documentation.

- Defining work, assigning responsibilities, and holding functional elements responsible for specific tasks or objectives. 
- Implementing project requirements and integrating project technical and programmatic activities with the project team and the APO, as appropriate.

- Developing a procurement strategy including project statements of work (SOWs), defining contracts, and monitoring contract negotiations in concert with ER Program Management, the responsible procurement contracting officer (buyer), and the APO.

- Controlling the project to ensure successful achievement of objectives.

- Conducting project reviews and preparing monthly status reports.

- Evaluating quality performance data from quality investigations, audits, and reviews related to the project on a periodic basis. Tracking reports on conditions adverse to quality, reviewing corrective actions, and tracking completion.

- Coordinating project activities and interfacing with the sponsor.

- Ensuring that project team comments to project documents are addressed and mutually resolved.

\section{MANAGEMENT OF ASSOCIATED LONG-TERM PROGRAMS}

Some ongoing environmental sampling programs have become part of ER operations. These programs may have a variety of reporting systems, but overall make some connection with ER through the Program Integration and Administration Division and possibly through the APO.

\section{ER QUALTTY ASSURANCE MANAGER AND SPECIAIJSTS}

The ER QA Manager and QA Specialists work with the ER project manager to ensure that project plans and necessary actions are taken to provide confidence that project objectives are met. The individuals are responsible for ensuring that items and services are defined and executed in accordance with applicable policies and directives. The ER QA manager and specialists are independent of ER management, reporting instead to Central QA.

Specific responsibilities include:

- Advising the ER Program manager and project team members on QA matters.

- Ensuring that QA requirements delineated by the ER Quality Program Plan (ES/ER/TM-4/R2), Energy Systems, and DOE are effectively implemented.

- Ensuring, through the ER Program manager and the subcontractor, that the QA Project Plan (QAPP) is adequately developed and effectively implemented.

- Identifying project QA requirements and assisting in the development of procedures and other implementing instructions, as required.

- Participating in the development, review, and approval of quality requirements contained in program procurement documents and other program documentation, as required. 
- Assisting in the identification of problems concerning the project or for unique project actions/events. Taking actions, as assigned, to eliminate or minimize potential problems (risk management).

- Reviewing and providing comments on program documentation such as SOWs, WPs, subcontractor proposals, and other project deliverables.

- Reviewing and commenting on subcontractor WPs, QA plans, standard operating procedures (SOPs), and other related documents and reports.

- Evaluating quality performance data from surveillances related to the project on a periodic basis. Tracking reports on conditions adverse to quality, reviewing corrective actions, and tracking completion.

- Conducting surveillances of subcontractor activities to determine compliance to QA requirements and associated procedures.

- Participating in preparation of the project audit schedule in concert with the APO, obtaining concurrence from the ER project manager, and assisting in planning, conducting, and reporting QA reviews/audits and follow-up activities, as required.

- Conducting quality investigations and participating in the review of corrective action plans.

- Providing QA training for project personnel.

- Assisting the ER Program manager in defining project QA documents and records and ensuring maintenance of project QA files.

- Coordinating all project QA activities and interfacing with sponsor and regulatory agency counterparts.

\section{ER TECHNICAL SUPPORT}

ER Technical Support consists of individuals responsible for providing technical direction and support to the project manager in specified areas such as hydrogeology, risk assessment, environmental engineering, and toxicology. Assistance may also be provided by Energy Systems Aralytical Chemistry Department (ACD) and AESG Sampling and Environmental Support Department personnel with regard to analytical sampling and $Q C$ areas.

Specific responsibilities may include:

- Ensuring that technical objectives are identified and achieved.

- Maintaining detailed knowledge of technical problems.

- Developing technical specifications and defining technical requirements.

- Reviewing and commenting on project technical documentation prepared for the project or by a subcontractor for accuracy and adequacy.

- Assisting in development of the project WP and DQOs.

- Developing technical sections of project documentation such as SOWs and WPs.

- Providing technical support to project audit teams, as requested. 
- Assisting in the identification of problems and taking actions, as assigned, to eliminate or minimize potential problems (risk management).

- Advising the ER project manager and project team members on QC matters concerning quality of environmentally related measurement data.

- Providing QC training for assigned project personnel.

- Providing technical support to the APO, as requested. AESG is presently designated by APO to review QA plans, lead audits, and send audit reports to recommended laboratories.

\subsection{SUBCONTRACTORS}

Subcontractors are responsible for providing specified technical support to the ER project manager. Responsibilities will vary based on the specific remedial actions process phase being addressed and the sponsor's project needs. The subcontractors' roles in the project will be defined in the planning process.

Specific responsibilities include:

- Identifying problems and initiating the implementation of corrective actions, if required (risk management);

- Implementing WP specifications (e.g., data validation); and

- Producing technical and project status reports.

\subsection{ANALYTICAL LABORATORY}

The analytical laboratory services are employed by the project directly (e.g., K-25 Site ACD) through: (1) an Energy Systems contract with a commercial laboratory or (2) by a subcontracted engineering firm. The laboratory must adhere to the laboratory requirements in this document.

Specific responsibilities include:

- Preparing and submitting an LQAP.

- Participating in an approved performance evaluation program (refer to Sect. 5.1.2).

- Submitting to on-site laboratory audits.

- Correcting any deficiencies cited in the LQAP, PE sample review, and laboratory audits.

- Identifying an LQAC responsible for overall QA. The LQAC position must fulfill the following requirements.

- Provides reports to the laboratory director.

- Is independent of project cost or profit responsibilities, schedules, or personnel, other than $\mathrm{QA}$ assistants. 
- Has the authority to stop work if QC problems arise affecting the quality of data produced.

- Submitting MPRs to the APO to maintain an active status in the program (see Sect. 5.1.4.1 for further information).

- Adhering to specific project QA plan requirements. The laboratory should have input into the development of these plans.

- Contacting APO if problems arise that prevent the laboratory from analyzing that they have accepted. 


\section{DATA QUALITY OBJECTIVES AND LEVELS OF QUALITY CONTROL}

\subsection{DATA QUALITY OBJECTIVES}

DQOs shall be developed in the project planning phase and prior to the initiation of data collection. DQOs define the needed sensitivity, precision, accuracy, representativeness, comparability, and completeness (SPARCC) of the data.

DQOs are statements of the uncertainty level a decision maker is willing to accept in results derived from environmental data. As such, they are a management tool used to limit the chance of data leading to an incorrect conclusion. The DQO process must also define the required level of data defensibility and hence the level of documentation desired. DQOs must strike a balance between time, money, and data quality. The DQO process (see Sect. 4) must be initiated during project planning to produce WPs resulting in data that have a quantifiable degree of certainty. The end use of data to be collected and the cost to produce that data will determine the required DQOs.

DQOs are specified in project documents such as the WP, the Sampling and Analysis Plan (SAP), and the QAPP. DQOs for the data generation activity must be defined prior to the initiation of field and laboratory work. Field and laboratory organizations must be aware of the DQOs to enable individuals to make informed decisions during the course of the project.

Five general levels of analytical options to support data collection are identified by CERCLA and have been adopted by ER to define DQOs. Analytical quality levels have been described in the U.S. Environmental Protection Agency (EPA) document Data Quality Objectives for Remedial Response Activities, Development Process, March 1987, EPA Office of Solid Waste and Emergency Response (OSWER) Directive 9355.0-7B (EPA 1987). These levels are based on the type of site to be investigated, the level of accuracy and precision require, and the intended use of the data. The level of $\mathrm{QC}$ and deliverables tends to increase with the level of DQO, although the relationship does not always hold true. The level of QC required at the site will be decided by the ER project manager in concert with the project team. Table 3.1 outlines analytical quality levels. These $\mathrm{QC}$ levels have been developed to represent a spectrum from qualitative to quantitative analysis which encompasses analytical survey methods through sophisticated methodologies. Table 3.2 outlines the basic ER QC and deliverables requirements for each level. Additional requirements may need to be tailored specifically to the DQOs for a given project, or the project may require more definitive DQO descriptors. The QC levels, like the DQOs, shall be developed before initiation of project work. Some program analyses require Level C QC but have a very low likelihood of being used in litigation and do not need to undergo data verification or validation. The most prominent example is the groundwater monitoring program. For such programs or projects, the project manager has the option to specify that QC forms do not need to be supplied as long as the laboratory is in house, the QC is actually performed, and the information is retrievable. This option is not appropriate for risk assessment or other investigative studies and is exercised at the discretion of the project manager. 
Table 3.1. Analytical quality levels and quality control levels

\begin{tabular}{|c|c|c|c|}
\hline $\begin{array}{c}\mathrm{AQ} \\
\text { level }\end{array}$ & Examples of data uses & $\begin{array}{c}\text { ER QC } \\
\text { level }\end{array}$ & Characteristics of data \\
\hline I & $\begin{array}{l}\text { Site characterization } \\
\text { Monitoring during implementation } \\
\text { Field screening }\end{array}$ & $\mathbf{A}$ & $\begin{array}{l}\text { Qualitative or semiquantitative } \\
\text { analysis } \\
\text { Indicator parameters } \\
\text { Immediate response in the field }\end{array}$ \\
\hline II & $\begin{array}{l}\text { Site characterization } \\
\text { Evaluation of alternatives } \\
\text { Engineering design } \\
\text { Monitoring during implementation } \\
\text { Field screening }\end{array}$ & B & $\begin{array}{l}\text { Semiquantitative or quantitative } \\
\text { analysis } \\
\text { Compound specific } \\
\text { Rapid turnaround in the field } \\
\text { May use an on-site laboratory }\end{array}$ \\
\hline III & $\begin{array}{l}\text { Risk assessment } \\
\text { Site characterization } \\
\text { Evaluation of alternatives } \\
\text { Engineering design } \\
\text { Monitoring during implementation }\end{array}$ & C & $\begin{array}{l}\text { Quantitative analysis } \\
\text { Compound-specific } \\
\text { Usually an off-site laboratory }\end{array}$ \\
\hline IV & $\begin{array}{l}\text { Risk assessment } \\
\text { Site characterization } \\
\text { Evaluation of alternatives } \\
\text { Engineering design }\end{array}$ & D & $\begin{array}{l}\text { Quantitative analysis } \\
\text { Compound-specific } \\
\text { Usually an off-site laboratory }\end{array}$ \\
\hline $\mathbf{v}$ & $\begin{array}{l}\text { Risk assessment } \\
\text { Evaluation of alternatives } \\
\text { Engineering design }\end{array}$ & $\mathbf{E}$ & $\begin{array}{l}\text { Qualitative to quantitative analysis } \\
\text { Method specific } \\
\text { Unique matrixes (e.g., pure waste, } \\
\text { biota, explosives) and nonapproved } \\
\text { methods }\end{array}$ \\
\hline
\end{tabular}

Note: $\mathrm{AQ}$ level $=$ Analytical quality level, $\mathrm{ER}=$ Environmental Restoration, and $\mathrm{QC}=$ Quality Control. 


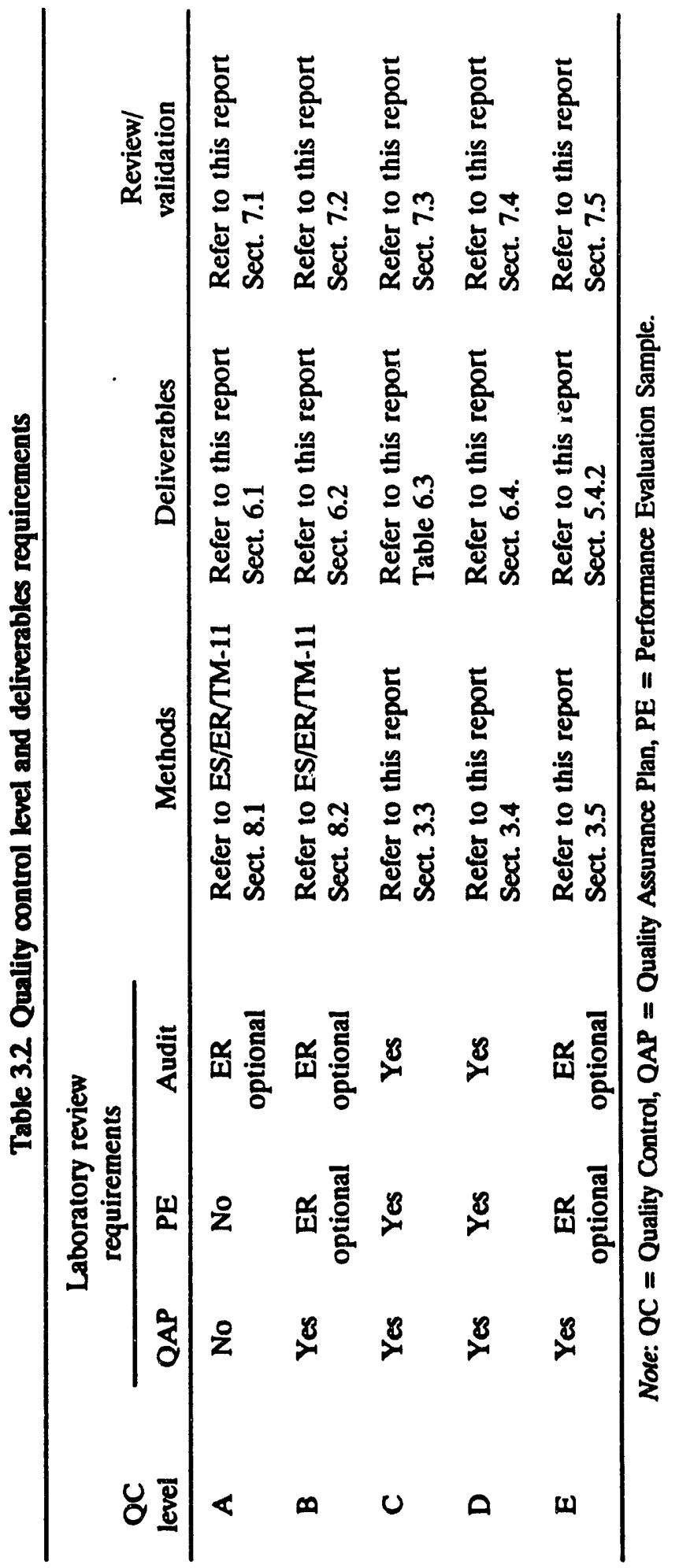


Laboratory method requirements for each level of QC are outlined in Sect. 5.5. QC requirements regarding performance sample analysis, laboratory audits, LQAP approval, and WP review do not change with the level. Levels of $\mathrm{QC}$ are for individual measurement activities, and more than one level may be used at a given site. Sections 3.2 through 3.5 describe Levels $A$ and $B$ relative to field measurements and Levels $C, D$, and $E$ relative to laboratory measurements; however, these descriptions are not intended to be so rigidly enforced as to preclude a particular QC level being employed at any physical location, as long as the specific requirements for that level have been met.

Project-specific DQOs must be developed based on the proposed end uses of the sampling and analytical data. The result of a properly executed DQO process will be a well thought out project WP providing the rationale behind the selection of specific sampling and analysis options and QC levels. The process should raise planning and design issues in an order and sequence that encourage thorough and effective consideration for the following questions.

- What are the overall project objectives?

- What decisions are required to reach project objectives?

- What data are necessary to resolve the decisions required to complete project objectives?

- What specific type and quality of data are needed to effectively resolve issues related to project decisions?

- How will data be used to make project decisions?

The DQO process should assist project management in establishing objective project criteria; ensuring the type, amount, and quality of data necessary to make project decisions; prespecifying levels of confidence in data; minimizing rework; and increasing effective communication among project team members.

\section{LEVELS A AND B QUALTYY CONTROL}

Levels A and B are designated for screening methods. Most often, by extension, these levels are specified for field analyses; however, they could be appropriate for laboratory screening as well. Typically the expense and time required to transport the sample to the laboratory makes field screening more desirable. The level of $\mathrm{QC}$ required for field analysis will be decided by the ER project manager in conjunction with the project team.

Applications of Levels A and B are based on the intended use of the data as stated in the site-specific project WP. Data usability may be restricted by inherent instrument limitations. It can also be restricted by how the instrumentation is used (e.g., a radiation counter that is set for a very short counting time). There are two basic types of field instruments: (1) qualitative and semiquantitative screening instruments (e.g., total organic vapor meters, calorimetric indicator tubes, $\mathrm{pH}$ indicator paper, radiological survey meters, and geophysical survey instruments) and (2) quantitative instruments that measure specific analytes, but often with less sensitivity than conventional laboratory units (e.g., portable gas chromatographs and portable $x$-ray fluorescence units). Qualitative and semiquantitative equipment will generally be governed by Level A criteria. Quantitative field instruments are 
usually governed by Level B criteria; however, Level A criteria may be sufficient. The level will be determined by the end use of the data.

Neither Level A nor Level B data alone can be used to dismiss a site. A representative percentage of all field sample results must be confirmed by sample analyses at Level C or D and must be supported by risk assessment. The number of sample analyses required by a nonfield laboratory will vary by site. The project WP must define the number or percentage of samples to be submitted for confirmation analysis. This is mandatory; confirmation is required regardless of whether field results are positive or negative. Applicable or relevant and appropriate requirements (ARARs) are often below the lower detection limits of field instruments; confirmation, therefore, is required to ensure that negative results are below the ARARs. Levels A and B QC are more fully explained in the ER document Requirements for Quality Control of Field Activities, ES/ER/TM-11 (Energy Systems to be issued in 1993).

\subsubsection{Level A Quality Control}

Data meeting Level A criteria are qualitative or semiquantitative in nature and are used as indicator parameters. Data are obtained by use of approved field equipment such as that given in the previous section. Other instruments and methods may be used if approved by the ER project manager.

Equipment capability, or the analytical QC implemented, will limit data obtained to qualitative, or at best, semiquantitative. Quantitative data are not obtained on an analytespecific basis. Level A data may be used for the following: (1) delineation of contaminated zones, (2) gross determination of analytes in samples, or (3) health and safety screening. Level A data can provide information to the in-house laboratory regarding expected concentration ranges. This information can assist the laboratory in determining applicable analytical ranges. Data are obtained immediately. For more information on Level A QC, see Requirements for Quality Control of Field Activities, ES/ER/TM-11, Sect. 8.1 (Energy Systems to be issued in 1993).

\subsection{Level B Quality Control}

Level B is also generally employed in field screening activities. It is, however, more quantitative than Level A. Level B QC will generally apply to on-site field laboratories conducting semiquantitative or quantitative analyses for rapid turnaround. Level B field instruments are more compact and rugged than traditional laboratory units but may be less sensitive than traditional laboratory instruments. Quantitative field instruments, which are designed for in situ measurements and do not require field laboratory support, are normally governed by Level B protocols. Note: Field laboratories can be designed to obtain Level C or D data. When generating Level $\mathrm{C}$ or $\mathrm{D}$ quality data, laboratories must meet all requirements as defined in this document, including undergoing the laboratory review process as defined in Sect. 5.2. Similarly, data obtained from instruments with quantitative capabilities may be employed for Level $A$, depending on the proposed use. In a few circumstances, Levels $C$ and $D$ data may be available from in situ measurements, and this capability is of strong interest. Appropriate QC must still accompany the sample analyses.

The QC level required must be determined before sampling and analysis begin. All analyses must meet requirements defined by the applicable QC levels. 
Data from Level B are used for site characterization, evaluation of alternatives, engineering design, and monitoring during implementation or sampling. For more information on Level B QC, see Requirements for Quality Control of Field Activities, ES/ER/TM-11, Sect. 8.2 (Energy Systems to be issued in 1993).

\subsection{LEVEL C QUALITY CONTROL}

Level CQC would be the minimum requirement at a site near a populated area requiring a risk assessment determination. Level $C Q C$ includes review of the project WP, including the SAF and QAPP. To perform Level C QC, the laboratory shall successfully undergo review of its LQAP, participation in an approved PE program (refer to Sect. 5.1.2), submit to audits, correct deficiencies found during audits, and provide MPRs. Level $\mathrm{C}$ provides low detection limits, a wide range of calibrated analytes, matrix recovery information, laboratory process control information, and kno:n precision and accuracy. EPA-accepted methods such as those in Test Methods for Evaluating Solid Waste, Physical/Chemical Methods SW-846 (EPA 1986), the National Pollutant Discharge Elimination System (NPDES), and the Contract Laboratory Program (CLP), are utilized under Level C. Nonstandard methods, or methods developed in support of a specific project or task, should be submitted to APO for approval prior to implementation.

Reference to the CLP forms later in this document is provided as an example of the type of information required from the laboratory. Because these forms are commonly used, are computerized, and present information essential to review data quality, they are referenced. It is recognized that sometimes it is necessary to use non-CLP methods such as SW-846 or NPDES to fulfill regulatory requirements, detection limits, required analytes, etc. CLP forms are not required as long as the same information is reported by the laboratory as would be required by the CLP methods. If forms other than CLP forms are used, the laboratory must include a copy of those forms in the LQAP or send them to the ER project manager for approval before initiating work.

The reason for using CLP is that QC specifics are mandated; for example, the exact surrogates that must be used are named. Other methods such as those described in EPA SW-846 or those described in the EPA 600 series of documents only recommend use of certain surrogates and allow changing or omitting them. If a newer version of the CLP becomes available, it may be appropriate to continue using the older version on the same project for purposes of data comparability; however, new projects should call for the latest version of the CLP.

In a few circumstances, Levels $C$ and $D$ data may be obtainable from in situ measurements, and this capability is of strong interest. Appropriate QC must still accompany the sample analyses.

Advantages of Level C QC are (1) greater precision and accuracy than Levels A and B and (2) more established and documented QC. Level $C$ can be used for risk assessment, but Levels A and B cannot. Level C may be used for (1) risk assessment, (2) site characterization, (3) evaluation of alternatives, (4) engineering design, and (5) monitoring during implementation. The time required to obtain data is typically 20 to 30 days. 
It is suggested that most laboratory soil and water analyses be performed using Level $\mathrm{C}$ QC. Level D should only be used at NPL sites, as required by regulators, or at sites where legal action is pending. At many sites, Level $\mathrm{C}$ laboratory data confirming the field screening data will be sufficient. Dependent on specific regulatory oversight, Level $C$ is applicable at NPL sites. However, it is recommended that a percentage of NPL site data be performed at Level D.

\subsection{LEVEL D QUALTY CONTROL}

Level D QC is used when comprehensive data quality documentation is required. This level is needed for select samples at NPL sites. These sites are typically near populated areas and are likely to undergo litigation. Unlike Level $C$, Level $D$ requires delivery from the laboratory of complete documentation, including all raw data. The CLP (or equivalent) protocol and full data package deliverables are required for all analytes. Methods not included in the CLP will be elevated to Level D by meeting CLP-type QC requirements and submitting all raw data.

Level D QC requirements are otherwise essentially equivalent to Level C QC. Requirements include review of the project WP, including the SAP and QAPP. The laboratory shall successfully undergo review of its LQAP, participate in an approved PE sample program (refer to Sect. 5.1.2), submit to audits, correct deficiencies found during audits, and provide MPRs.

Also like Level C, Level D QC provides low detection limits, a wide range of calibrated analytes, matrix recovery information, laboratory process control information, and known precision and accuracy limits.

Advantages of Level D QC include acceptance by all EPA state and regional offices and other regulatory agencies. The required submission of standardized data reporting forms and all raw data allows for the most complete data quality assessment and defensibility.

Level D data may be used for (1) risk assessment, (2) site characterization, (3) evaluation of alternatives, (4) engineering design, and (5) monitoring implementation. The data are typically obtained in $\mathbf{3 0}$ to $\mathbf{4 0}$ days.

\subsection{LEVEL E QUALTYY CONTROL}

Level E QC is used for analysis of unconventional sample matrixes such as air, biota, and pure waste. It may also be employed for unconventional methods such as those for explosives. Level E QC is also appropriate for analysis of the contents of underground storage tanks, where samples are primarily pure product or waste. This document defines the minimum QC requirements for Level $E$; however, specific $Q C$ requirements may vary among sites. Specific QC requirements must be clearly and completely identified in the project WP when Level $E$ is employed.

As part of the project plans, all analytical methodologies will be clearly defined. The approval of the plans will constitute approval of the analytical methods for the intended use-EPA approved and non-EPA approved methods. On a case-by-case basis, approval of the 
methodologies will be sought through meetings and conversations. All conversation and meetings will be documented in a letter of conversation. All letters of conversation will be sent to all responsible parties, and a copy will be placed in the project file for permanent documentation. A response to the letters of conversation is not required from the parties involved.

Level E QC includes review of the project WP, including the SAP and QAPP. If requested by ER, the laboratory shall successfully undergo review of its LQAP, participate in an approved PE sample program (refer to Sect. 5.1.2), submit to audits, correct deficiencies found during audits, and provide MPRs. Methods to be used for Level $\mathrm{E}$ analysis must be submitted to the ER project manager for review and approval before initiation of work.

Because few methods are available for unconventional matrixes, some methods must be developed along with precision and accuracy information. Method development is often time consuming and costly; therefore, project schedule must be planned accordingly.

Level E data may be used for (1) risk assessment, (2) site characterization, (3) evaluation of alternatives, (4) engineering design, and (5) monitoring implementation. 


\section{PROJECT REQUIREMENTS}

ER work may be oriented to monitoring programs, investigative projects, or miscellaneous characterizations. This section deals with projects. Once the project team (as described in Sect. 2) is assembled, the project moves through an orderly series of events toward successful completion. Some of these events, related to analytical QC requirements, are addressed in this section.

\subsection{IDENTIFICATION OF DATA QUALITY OBJECTIVES}

One of the first and most important decisions is the identification of DQOs. Directions for this decision can be found in Data Quality Objectives for Remedial Response Activities, Development Process (EPA 1987). DQOs shall be defined and QC levels selected prior to project work proceeding.

DQOs are developed through a three-stage, interactive, continuous-thought process. The first stage is to identify suspected sources, contaminant pathways, and potential neceptors and to use this information to specify decisions. The second stage is to identify data needed to make project-related decisions and to use this information in the selection of sampling approaches and analytical options for the site. This decision is weighed against the cost and time of data collection evaluation. The third stage is to design the data collection program so that data of acceptable quantity and quality will be generated from which decisions can be made.

There are many considerations to make during this development process. Some of these considerations have been outlined by the DOE Environniental Management proposed DQO process as follows:

Stage 1: Define the problem scope.

- Determine the nature of the problem.

- Determine analytical parameters of concern.

- Determine suspect sources, contaminant pathways, and receptors.

- Evaluate resource allocations, including time, budget, equipment, labor, and space.

- Identify and evaluate new methodologies or technologies that may be applicable.

- Identify appropriate ARARs.

Stage 2: Establish campling and analytical data requirements, including data quality needs.

- Evaluate the ability of existing data to meet project objectives.

- Identify the intended data uses, including health and safety, risk assessment, site characterization, site remediation, performance of remedial technologies or methods, and enforcement or regulatory concerns. 
- Establish evaluation measures for the sampling and analysis, SPARCC, especially the level of detection of sensitivity. The required defensibility of the data also must be established.

Stage 3: Design the data collection program.

- Develop a sampling approach, including statistical considerations, location, number of samples needed, frequency, and methods.

- Develop an analytical protocol, including defined QC levels and analytical methods.

Once the DQO process has been completed, the general framework for the WP, SAP, and QAPP will have been established.

\section{DEVELOPMENT AND APPROVAL OF THE PROJECT WORK PLAN}

The project WP documents decisions and evaluations made during the scoping process and provides a framework for addressing the identification of and subsequent actions required to address environmental project problems. The objective of the plan is to provide a detailed technological structure for addressing identified sites and for accumulating data of sufficienc quantity to at least support completion of a final report. Data collected must be of sufficient quality to support future project planning and to support the necessary activities associated with the chosen approach, including a risk assessment or decision document. Guidance for preparing WPs may be found in Guidance for Conducting Remedial Investigations and Feasibility Studies Under CERCLA (Interim Final) (EPA 1988a), OSWER Directive 9355.3-01. The DOE/Energy Systems Document Content and Response Committee (DCRC) is developing annotated outlines that specify formats for certain types of documents. The following outlines are completed:

- Preliminary Assessment/Site Inspection

- Remedial Investigation Work Plan

- Remedial Investigation Report, including Baseline Risk Assessment

- Feasibility Study

- Interim Proposed Plan

- Interim Record of Decision

- Record of Decision

DOE-OR is preparing a procedure that will mandate use of these outlines for all its ER contractors. Energy Systems is preparing a comparable procedure for internal use. The annoted outlines are available from the following individuals:

- Oak Ridge Associated Universities Document Conte:t and Response Committee chairperson, DOE-OR, 576-2552

- Federal Facility Agreement compliance manager, Martin Marietta Energy Systems, Inc., 576-4025 
The DCRC outlines meet the guidelines given in the OSWER directive. General content guidance is given below; however, this information is not a substitute for the DCRC guidelines.

WPs usually consist of the following:

- Work Plan-Provides an overall technical strategy and management approach for completing investigations.

- Sampling and Analysis Plan-Consists of two parts: (1) the Field Sampling Plan (FSP) that provides guidance for all fieldwork by defining, in detail, the sampling and datagathering methods to be used and (2) the QAPP that describes the policy, organization, functional activities, and QA and QC protocols necessary, including laboratory QA and $\mathrm{QC}$, to achieve the DQOs dictated by the intended use of data.

- Health and Safety Plan (HASP)-Identifies site-specific measures for ensuring worker health and safety. This document must reflect the health and safety program of subcontractors on site, which must be in compliance with the Energy Systems' Health and Safety Program and the Occupational Safety and Health Act.

- Community Relations Plan (CRP)-Documents the community relations history and the issues of community concern.

These plans may be submitted as a single document, although they are more easily used in the field if bound separately.

Typical content for each plan is described herein. Other requirements may be imposed by the ER project manager, based on project needs.

\section{Suggested Content of Work Plans}

The content and format of work plans will be developed by the DCRC. The content will include the following:

- Executive Summary

- Introduction

- Site Background and Setting

- Initial Evaluation

- Rationale

- Tasks

- Costs and Key Assumptions

- Schedule 
- Project Management

- References

- Appendixes

\subsection{Suggested Content of Sampling and Analysis Plans}

The content of Part 1, the FSP, of the SAP follows.

Site Background-A summary of existing data, a description of the site and surrounding areas, a discussion of known and suspected contaminant sources, and a listing of probable transport pathways.

Sampling Objectives-A description of intended data uses.

Sampling Location and Frequency-Identification of each sample matrix to be collected, identification of the constituents to be analyzed, maps and/or drawings identifying the location of sampling points, and summary tables showing numbers of samples by matrixes and sites.

Sample Designation-A description of the sample numbering system.

Sampling Equipment and Procedures-A description of sampling procedures, including equipment to be used and material composition of the equipment; a detailed description of decontamination procedures; a discussion of mobilization and demobilization; a detailed description of, and procedures for, field screening methods, including preventive maintenance; a discussion of surveying wells and sampling points; a detailed description of water-level measurement procedures; a detailed description of borehole and well drilling methods; a detailed description of piezometer and monitoring well installation procedures, construction design, and materials; and a detailed discussion of well development and purging methods.

Sample Handling and Analysis-Identification of sample holding times, preservation methods, types of sample containers, and volumes of samples to be collected; shipping requirements and procedures; COC procedures; disposal of wastes generated; and a discussion of field logbooks/forms/notebooks, including how to complete them and how they are controlled.

The content of Part 2, the QAPP, of the SAP follows.

Title Pago-Page for signatures of approval personnel, including the subcontractor project manager and QA manager.

Table of Contents-Outline of report.

Project Description-A general site history, objectives of the investigation, and the site description.

Project Organization and Responsibilities-Organizational chart identifying key personnel and organizations and responsibilities of key personnel. 
Quality Assurance Objectives for Measurement-Intended data use; a listing of method detection limits; a table of QC samples (duplicates, trip blanks, source water blanks, and equipment rinsates) versus the number of samples by method and matrix (include extra sample volumes for QC samples); a detailed discussion of DQOs, including how they will be implemented; and a table, broken down by site, showing the analysis method, method number, sample media, DQO level, and number of samples.

Sampling Procedures-A description of sampling procedures; a discussion of the cleaning/ preparation of sample containers; a description of sample preservation techniques and holding times; a discussion of field logbooks/forms/notebooks; and a discussion of material blanks, materials certification, and readiness review.

Sample Custody-COC procedures.

Calibration Procedures-Written field calibration procedures, including frequency of calibration, source of calibration standards, and calibration acceptance criteria; a detailed discussion of accuracy and precision of field instruments; and a detailed discussion of the field data evaluation process.

Analytical Procedures-Tables of analyses method numbers, numbers of analyses per matrix for each site, the name of the analyte list, and a list of analytes for multi-analyte methods.

Data Reduction, Validation, and Reporting-The principal criteria used to validate data, a detailed discussion of data handling and reduction procedures, methods for evaluation of blanks, and QC acceptance criteria.

Internal Quality Control-Discussion of matrix spike/matrix spike duplicates (MS/MSDs), field duplicates, source-water blanks, trip blanks, equipment rinsates, and surrogates; and identification of ways in which the QC information will be used to qualify data.

Performance and Systems Audits-A discussion of performance and system audits to be performed.

Preventive Maintenance-Discussion of preventive maintenance, including critical spare parts.

Data Assessment Procedures-Discussion of SPARCC parameters and statistical applications of data (subcontractor responsibility).

Corrective Actions-A discussion of corrective action procedures, including field changes and responsibilities for corrective actions, and a discussion of out-of-control conditions reporting and follow-up procedures.

Quality Assurance Reports-Results of audits, significant QA problems encountered, and recommended solutions; a discussion of project deliverables, including laboratory deliverables, MPRs, and the final report and its contents; a summary of final data quality; and summary tables of the data. (Table 4.1 contains a sample portion of a data summary table to show the format to be followed.) 
Table 4.1. Sample portion of data summary tables

\begin{tabular}{|c|c|c|}
\hline $\begin{array}{l}\text { Sample No.: } \\
\text { Lab Sample No.: } \\
\text { Matrix: } \\
\text { Associated Samples: }\end{array}$ & $\begin{array}{l}\text { S3-MW1 } \\
890321-11 \\
\text { Water } \\
\text { TB-3 } \\
\text { FB-2 } \\
\text { ER-c } \\
\end{array}$ & $\begin{array}{l}\text { S3-SS6-6.0' } \\
890321-17 \\
\text { Soil } \\
\text { TB-7 } \\
\text { FB-4 } \\
\text { ER-9 }\end{array}$ \\
\hline Volatile organics & $\mu g / L$ & $\mu \mathrm{g} / \mathbf{K g}$ \\
\hline Chloromethane & $10 \mathrm{U}$ & $10 \mathrm{UJ}$ \\
\hline Bromomethane & $10 \mathrm{U}$ & $10 \mathrm{UJ}$ \\
\hline Vinyl chloride & $10 \mathrm{U}$ & $10 \mathrm{UJ}$ \\
\hline Chloroethane & $10 \mathrm{U}$ & $10 \mathrm{UJ}$ \\
\hline Methylene chloride & $13 \mathrm{~J}$ & $5 \mathrm{UJ}$ \\
\hline Acetone & 36 & $26 \mathrm{~J}$ \\
\hline Carbon disulfide & $5 \mathrm{U}$ & $5 \mathrm{UJ}$ \\
\hline 1,1-Dichloroethane & $5 U$ & $5 \mathrm{UJ}$ \\
\hline 1,1-Dichloroethane & $5 U$ & $5 \mathrm{UJ}$ \\
\hline 1,2-Dichloroethane (Total) & $5 \mathrm{U}$ & UJ \\
\hline Chloroform & $5 \mathrm{U}$ & UJ \\
\hline 1,2-Dichloroethane & $5 U$ & UJ \\
\hline 2-Butanone & $6 \mathrm{~J}$ & $10 \mathrm{UJ}$ \\
\hline 1,1,1-Trichloroethane & $5 U$ & $5 \mathrm{UJ}$ \\
\hline Carbon tetrachloride & $5 U$ & UJ \\
\hline Vinyl acetate & $10 \mathrm{U}$ & $10 \mathrm{UJ}$ \\
\hline Bromodichloromethane & $5 \mathrm{U}$ & $5 \mathrm{UJ}$ \\
\hline 1,2-Dichloropropane & $\mathrm{U}$ & UJ \\
\hline cis-1,3-Dichloropropene & $\mathrm{U}$ & $5 \mathrm{UJ}$ \\
\hline Trichloroethene & 7 & $14 \mathrm{~J}$ \\
\hline Dibromochloromethane & $\mathbf{U}$ & $5 \mathrm{UJ}$ \\
\hline 1,1,2-Trichloroethane & $\mathbf{U}$ & UJ \\
\hline Benzene & $\mathbf{U}$ & UJ \\
\hline trans-1,3-Dichloropropene & $\mathrm{U}$ & UJ \\
\hline Bromoform & $5 \mathrm{U}$ & UJ \\
\hline 4-Methyl-2-pentanone & $10 \mathrm{U}$ & $10 \mathrm{UJ}$ \\
\hline 2-Hexanone & $10 \mathrm{U}$ & $10 \mathrm{UJ}$ \\
\hline Tetrachloroethene & $5 U$ & $5 \mathrm{UJ}$ \\
\hline 1,1,2,2-Tetrachloroethane & $\mathrm{U}$ & UJ \\
\hline Toluene & $\mathbf{U}$ & UJ \\
\hline Chlorobenzene & $\mathbf{U}$ & UJ \\
\hline Ethylbenzene & $\mathbf{U}$ & UJ \\
\hline Styrene & $\mathbf{U}$ & UJ \\
\hline Xylene (Total) & $5 \mathrm{U}$ & UJ \\
\hline
\end{tabular}

Nore: $\mathrm{U}=$ quantitation limit, $\mathrm{J}=$ estimated value, $\mathrm{UJ}=$ estimated quantitation limit. 


\subsection{DATA REVIEW REQUIREMENTS}

Data review is composed of four steps.

- Laboratory data review

- Compliance screening

- Data validation

- Data assessment

The laboratory data review step is done by the laboratory performing the analyses. During this step, the laboratory reviews data to verify that it has met the requirements of the method. The laboratory shall generate a case narrative describing deviations from method and discrepancies between the method performed and the method required. The laboratory will verify that all laboratory deliverables have been provided for the project. The completed and reviewed data package goes to the engineering firm, APO, or designated entity for use in compliance screening.

Compliance screening is a review of the data package to verify that the laboratory met laboratory project deliverables and the field team met field project deliverables. Review of laboratory deliverables shall include a review of data to verify that the laboratory met the requirements of the analytical method and laboratory project deliverable requirements. The review also includes the deliverables required from the field effort and verification that all are present, as required by the project. Discrepancies in deliverables shall be resolved at this point and incompleteness of data shall be noted. This information shall be supplied to the data validator. Completed and reviewed laboratory and field project deliverables goes to the data validator for validation.

As listed in the aforementioned requirements, the project shall indicate in the sitespecific QAPP the systematic process to be used to validate project data. Data must be validated against a set of accepted criteria to provide assurance that data are adequate for their intended use (i.e., that project-specific DQOs are met). The APO reviews the DQOs and validation criteria to ensure that the validation criteria are adequate for the intended use of the data.

The validation process is a technical review of the data. The intensity of the review, the types of checks performed, and the degree of reviewer independence from other parties involved are project specific. At a minimum, validation includes checking the accuracy of data transfer, interpretation of raw data, and laboratory review; identifying factors from compliance screening which affect data values; flagging data based on factors external to the laboratory (such as whether the source water blank or equipment rinsate shows contamination); and certifying the data. Validation may also include reviewing the reasonableness of the data in terms of a model, related sites, or other criteria.

The project must specifically designate a representative to perform data validation. The analytical laboratory shall not perform data validation; validation is independent of the analytical laboratory data review. The project shall certify in writing that data have been validated and flagged in accordance with the defined process. Specific guidelines per QC level are presented in Sect. 7. The APO has oversight of validation activities. 


\subsection{PROJECT FINAL REPORT}

A draft of the final report shall be produced by the ER project manager and technically reviewed before its release. This report is the final deliverable for the project. It shall be designated as an ER QA record. A typical report includes the following. Again, the DCRC guidance will provide a specific format.

- Title identifying the project name or contract number.

- Foreword signed by those with major responsibilities for the QA program and by project management.

- Executive Summary presenting a brief review of the report and a site description.

- Table of Contents with approximately the same level of specificity as the Table of Contents in this ER document.

- Introduction that summarizes the ER project (sites of interest, dates of sampling, and dates of analyses), including objectives of the QAPP as they relate to the study.

- Data Summary that provides a synopsis of results on a site-by-site basis (see Table 4.1).

- Additional Information, including presentation of other requested information from the SOW such as risk assessment, recommendation for continued site characterization, or recommend site closure. This information was specified before beginning work and is directed by the ER project manager.

- Findings from the analytical data. As stated previously, blank subtraction is not allowed. Data will be flagged as per data validation guidelines; all data validation flags will be included with results of the final data.

- QC Summary including a discussion of all flagged data. Validation notes, including flagged data (defined as data for which trip, source water, or laboratory blanks were contaminated, MS/MSDs exceeded limits, surrogate recovery criteria were exceeded, calibration criteria were not met, and LCS recoveries exceeded acceptable limits) will be included. The QC summary will discuss results of laboratory blanks, MS/MSDs, duplicates, control charts, surrogate recoveries, holding times, source-water blanks, trip blanks, equipment rinsates, and field duplicates. This section will also discuss SPARCC parameters, QC frequency, audits, corrective actions, and holding times.

- Investigation activities shall include (where applicable) a discussion of drilling methods; decontamination waste disposal; well installation, development, and purging; sampling methods; water-level measurements; geophysical testing; field screening; surveying; and custody and shipping of samples. These items must be addressed when they differ from those in the WP.

- The report shall indicate the duration and location of storage for data. Stored data will consist of all raw data, QC charts, corrective actions, logs, sample lists, COC information, notebooks, work sheets, automated data processing system output, calibration information, and validation notes. 
- All field and analytical data must be presented. The preferred means to accomplish this is through use of appendixes, as described below. When the volume of data is massive, the following alternatives may be considered if the customer and regulatory interests approve:

- Reference may be made to the document or other source that contains the data, provided the source is readily available to potential data users. For the deliverable report, the source document may need to accompany the report.

- Data may be delivered on disk if disks can be made available to potential data users.

One appendix shall contain field logs and forms. A second appendix shall contain the laboratory data for each sample. All trip, field, and laboratory blanks shall be marked allowing for sample and blank association. For Level C QC, deliverables as discussed in Sect. 5 shall be presented. For Level D QC, the laboratory shall submit full CLP or CLP-type data packages. Control charts for LCS data will be submitted. For Level E QC, sample results, initial and continuing calibration forms, method blanks, and LCS charts are required. Exact deliverables will have been stated and approved in the project WP.

A third appendix shall include LCS control charts, surrogate recoveries, MS and duplicate analysis, field and laboratory duplicates for all spike samples, and any additional QC analyses associated with the project.

A fourth appendix shall include all validation notes, as appropriate, to the QC level defined for the project. 


\section{SPECIFIC ENVIRONMENTAL RESTORATION REQUIREMENTS}

Prior to and throughout the duration of any field studies or analyses of samples, contracted laboratories must fulfill ER requirements. This section describes the basic requirements that laboratories providing analytical support to ER must satisfy. These requirements may be categorized as:

- Review requirements

The laboratory must satisfy the ER requirements for the Laboratory Review Program. The pre-award requirements include four elements:

- Acceptable LQAP

- Satisfactory submission of PE data from an approved PE program (refer to Sect. 5.1.2)

- Laboratory inspection and audit

- Cost-effectiveness and other procurement requirements

Postaward requirements include:

- Satisfactory periodic requalification (requirements as for pre-award review)

- Submission of analytical MPRs outlining progress of the analysis and related QC aspects

- Operational requirements

Laboratories must implement the ER requirements regarding organization and personnel, SOPs, holding times, sample receipt, COC, error correction, cleanness of sample containers, out-of-control events, document control, laboratory control samples, and control charts.

- Analytical requirements

Samples related to ER projects must be analyzed using approved analytical methods. Methods not listed must be reviewed and approved by the APO prior to use.

Failure to comply with any of the requirements listed in this section may result in the suspension of the laboratory from participation in ER programs or projects. 


\subsection{LABORATORY REVIEW PROGRAM REQUIREMENTS}

The program applies to all laboratories providing analytical support for the ER Program. The program is designed to ensure that contracted laboratories maintain an internal QC program that facilitates the generation of data that are of known quality and are both traceable and defensible. Objectives of the approval process are

- to ensure that proper communication and planning between the project and laboratory has occurred before the laboratory receives samples related to ER projects,

- to verify ER QC requirements are being met by each laboratory before analysis of ER samples, and

- to establish a plan for maintaining the QC program while work is being performed for any ER project.

Analytical laboratories associated with the project must be identified as early as possible in the project planning process. APO begins the review process of the designated laboratories.

The APO will define a laboratory review process. Components of the review process are described in the remainder of Sect. 5.

\subsubsection{Laboratory Quality Assurance Plan Requirements}

The LQAP is a statement of the laboratory's approach to ensure that quality data are generated. The LQAP will be forwarded to the APO for review. If the project manager wishes to also review the LQAP or to have a separate copy of it, that should be indicated to the APO with the request for laboratory review. It is the policy of APO that the LQAP must be submitted within 2 weeks after receipt of the written request for review. Deficiencies or deviations from ER requirements will be identified by the APO in a written correspondence to the laboratory. The laboratory must correct all deficiencies prior to the beginning of any analysis associated with the project. Corrections may be in the form of a revised LQAP, attachments or addenda to the LQAP, or a written response outlining the deficiency and the corrective action to be implemented by the laboratory. All changes or plans of action must be in effect before laboratory approval.

The following items are required in the LQAP; however, they may be presented in any order that the laboratory desires. The requirements are based on and must be consistent with DOE Order 5700.6C, ANSI/ASQC standards, and QAMS-005/80.

- Title Page with Provision for Signatures-A title page with provisions for approval signatures and dates of revision will be provided.

- Table of Contents-A detailed Table of Contents will be provided.

- Laboratory Organization and Personnel-Overview of the laboratory organization as it relates to implementation of the QC program. The roles, responsibilities, and authorities of key laboratory personnel are described, with emphasis on the authority given the LQAC regarding QC monitoring, reporting, and corrective action. An appendix will contain a list of all personnel, their assignments and responsibilities, degrees of 
education, and years of applicable experience. This information may be supplied in the form of resumes. Any education and training related to tasks performed for this project will also be listed.

- Personnel Training-A discussion of personnel training in laboratory analytical methods, QC procedures, and safety policies. Frequency of training and training records will be addressed.

- Sample Management Practices-Provide procedures for receipt of samples, verification of preservation, log-in of samples, documentation, and tracking samples through the laboratory. Sample storage and disposal procedures will also be included along with methods of preparing bottles and cleaning glassware.

- Holding Times and Preservatives-A discussion of the holding time policy for ensuring sample analyses protocols are met. Sample storage, holding times, and preservatives specified by the methods are minimal criteria for the ER projects.

- Material Procurement and Control-A description of procedures for purchasing materials, quality inspection before use in sample analysis, chemical standard inventory procedures, solvent storage policies, and laboratory waste disposal.

- Facilities and Equipment-A list of basic equipment types, models, and years and a general description of the facility.

- Equipment Maintenance-General information as to who performs major, preventative, and day-to-day equipment maintenance and how it is documented.

- Analytical Procedures-A list of procedures the laboratory offers by method number and matrix. Any method variances employed by the laboratory must be documented. Documentation for EPA method variance approvals will be presented.

- Calibration-Calibration procedures by instrument type. Calibration frequency, reference standards, calibration acceptance criteria, and calibration documentation procedures must be addressed. Procedures must be defined for ensuring that balances, refrigerators, ovens, and other laboratory equipment are accurate and that their performance is monitored and documented. Balances must be checked each day they are used and must be calibrated annually by an independent company. Refrigerator temperatures must be monitored daily.

- Limits of Detection-A listing of the typical method-detection limits achieved for water, soil, and other matrixes commonly analyzed by the laboratory. It is understood that these may vary with individual samples; however, procedures for determining limits of detection and the frequency of verification will be outlined.

- Analysis of QC Samples and Documentation-A summary of QC procedures and documentation to be employed in the day-to-day operation of the laboratory. The discussion will emphasize the following as they relate to the different QC levels:

- Analysis of method and reagent blanks. 
- Analysis of duplicates, spiked samples, spiked laboratory blanks, and reference or control standards such as EPA check standards.

- The criteria used to establish warning and control limits for the above types of QC samples.

- Documentation and examples of control data and control charts (see Sects. 5.4.1 through 5.4.4 for an explanation of control charts and their usage).

- The frequency of blanks and other QC samples.

- How data from QC samples are reported and reviewed.

- Who reviews and makes decisions relative to QC data.

- Out-of-Control Events and Corrective Action-This section will define types of out-ofcontrol occurrences, how these occurrences are documented, and who is responsible for correction, documentation, and follow up action. Some examples of out-of-control events and corrective action are:

- Structural flaws in electronic data package deliverables, when the requirements of the program requires electronic deliverables.

- Corrective actions at the receiving level-A sample is broken during transport. The occurrence is documented on an out-of-control form. Corrective actions include notification of project management, who will determine the need for resampling.

- Observations corrected at the bench-Calibration of an instrument is not linear. The analyst finds this and corrects the problem before continuing sample analysis. The laboratory must document this, note that the corrective action was to recalibrate, and document that no samples were affected.

- Corrective action taken by analyst-A semivolatile organic analysis sample exhibits matrix problems or is damaged. The analyst must request re-extraction by submitting a written re-analysis request form or comparable documentation to the preparation laboratory.

- Corrective actions taken by supervisor-An MS recovery is out of control, and the laboratory supervisor discovers this after samples have been analyzed. The supervisor must document the occurrence and the corrective actions taken.

- Statistical out-of-control events-A control chart is being monitored, and the measured parameter exceeds control limits. The occurrence is documented on an out-of-control form, the root cause is established, affected samples are identified, and corrective actions are defined.

The laboratory must specify protocols for reporting any incident that delays sample processing for a period of time, affects holding times, or delays work and must specify the corrective action implemented. Examples of forms used to document out-of-control events are to be provided in the LQAP.

- Corrective Action Reports-Out-of-control incidents and corrective actions should be thoroughly documented to prove the system is back "in control." 
- Document Control-The LQAP will outline document flow from the COC to the final data. The LQAP will explain how documents are reviewed, signed, and filed. The laboratory policy and its implementation will ensure that controlled copies of analytical procedures and SOPs are available to the analyst.

- Data Review and Reporting-A discussion of data evaluation procedures for each analytical method, as well as for an entire data set, will be included. The process for da:a review and approval will be outlined as will the process for identifying the person with authority to release the data. Data qualification and flagging procedures will be implemented.

- Internal Laboratory Audits and Approvals from Other Agencies-The document will include a listing of approvals from other agencies and states. When the laboratory performs self-audits, frequency and method of documentation will be outlined.

- QA Reports to Management-The plan will include the frequency and information (general contents) of QA reports to management.

- Accuracy, Precision, Completeness, and Representativeness-The plan will include the laboratory's definition of accuracy, precision, completeness, and representativeness. The method for evaluating measured parameters and data sets for accuracy, precision, completeness, and representativeness will be incorporated.

\subsubsection{Performance Evaluation Sample Requirements}

For approval, the laboratory must have successfully participated in an external performance evaluation program for analytes representative of those anticipated in environmental samples. The purposes of PE sample analysis are to (1) gauge each laboratory's proficiency by providing samples designed to mimic field samples and (2) provide a known material from a source outside the laboratory which can be used to evaluate performance.

The laboratory's participation in such programs must continue for the duration of its ER work. Acceptable PE programs include the EPA CLP quarterly blinds; drinking water (WS), water pollution (WP), and Environmental Monitoring and Support Laboratory (Las Vegas) Radioactivity Laboratory Intercomparison Studies; and Environmental Measurements Laboratory cross checks. The APO will determine the acceptability of other PE programs for meeting this requirement.

The laboratory's PE sample results must be submitted to the APO for evaluation before beginning ER work. Subcontracted analysis of PE samples is not allowable.

\subsubsection{Initial Laboratory Audit Requirements}

A laboratory audit will be conducted after the following events take place.

- The LQAP is reviewed.

- The laboratory has satisfactorily responded to LQAP review comments.

- The laboratory has submitted the required PE data. 
The purpose of a laboratory audit is to verify that ER QC requirements are being implemented, as reflected by the laboratory's daily operations in adherence to the LQAP.

\subsubsection{Laboratory Inspection Process}

Before the laboratory inspection takes place, the auditors prepare an audit plan, including lines of inquiry and checklists. The laboratory inspection involves three phases.

Pre-audit Meeting (Uverview and Orientation)-The audit team meets with laboratory' management, including the laboratory director, the LQAC, and others, as the director deems appropriate. Objectives of the visit are reviewed, and a schedule is established. The audit team discusses comments on the LQAP and PE data. Basic requirements, as outlined in this documant, are discussed. Laboratory personnel provide information on training, the laboratory's history, and capabilities for performing work for ER. Project- and programspecific requirements may be discussed.

Observation, Examination, and Review (Isaboratory Walk-Through)-The audit team. performs the following activities:

- Reviews sample receiving, handling, and storage procedurer. The audit teata will follow the paper trail of the PE or utiar ER sarm les through the laboratory.

- Witnesses performance of anaiytical procedures in each section of the laboratory.

- Examines QC records, including manuals, instrum:nt calibracion records, run logs and maintenance records, sample preparation logss, and notebocks used to document analyses. Other $Q C$ records such as control charts, correcuve action reports, and ferformance data generated for other programs such as CLP and state drinking water programs will be examined. SOPs for all activities performed mov be reviewed. Other itcatic examined are waste disposal procedures; water sources; bottle preparation activities; records for balances, refrigerators and ovens; data review procedures; and internal eudit proceciures and reports.

Cose-out Meeting (Exit Interview)-The audis team will conduct an exit interview with the laboratory director, LQAC, and any other laboratory personnel the direcior deems appropriate. The audit tearn will summarize findings and detail specific deficiencies and possible corrective actions. A written report summarizing audit findings is provided to the LQAC within 10 working days of the inspection.

\subsubsection{Corrective Action (if Required)}

Within $\mathbf{1 0}$ working days of receipt of audit findings, the laboratory must submit an action plan to the APO. The plan will include, for each deficiency, a description of the corrective action and completion date. If applicable, required funding, responsible organizations, and root causes are included. All corrective actions must be in place or initiated within 45 working days of audit report receipt or prior to start of the project, whichever comes first.

The laboratory may be requested to submit i follow-up report that supplies information indicating that the plan of action has been implemented. For exanple, if no control charts 
exist, the plan would state that these would be in place by a specific date, and the follow-up report would contain copies of the control charts.

\subsection{Follow-up Audits}

A follow-up audit may be required in instances where deficiencies requiring corrective action are complex. Follow-up audits will be scheduled for the earliest possible date after the last corrective action plan is received by the APO.

\subsubsection{Notification of Status}

Upon acceptance of the LQAP and PE sample data and resolution of all audit findings, the APC will send written notification to the laboratory, ER, and other key participants stating that the laboratory has met requirements for participation in the program.

\subsubsection{Continuing Laboratory Reviews}

\subsubsection{Laboratory Monthly Progress Report Requirements}

The overall objective of the laboratory MPR is to maintain a continuing communication between the APO and those laboratories which have successfully passed the laboratory review process. It will be a means of transmitting information concerning personnel, systems, and structure of the laboratory, and will assist in monitoring communication between project field operations and the laboratory. In addition, the MPR will provide a continuous observation of laboratoly performance. The purpose of the MPR is not to monitor specific project data quality.

Once received by the APO, information will be distributed to appropriate personnel and reviewed, with comments forwarded to the laboratory. Laboratory responses to comments on MPR. rill be, included in the next MPR.

This report is not a project-specific deliverable unless defined as such in the project WP. It is a requirement to maintain a continuing satisfactory review status. The information requested should be readily available in the laboratory records and should not require significant effort to copy, collate, and transmit to the APO.

Each laboratory is expected to submit only one MPR per month. An MPR must be submitted each month, even when ER samples are not in the laboratory. MPRs are to be submitted by the laboratory to the APO by the 15th of each month, reflecting activities periormed during the preceding month. The following information is to be included in the MPR.

Reporting Period-The time frame encompassed by information presented in the report must be identified. This is expected to be at a consistent 1-month interval, from the beginning to the end of each month. The MPR should be received by the APO by the 15 th of the month following the reporting period. The project should be identified, if possible, and the agreement with the APO, subcontracted engineering firm, or othcs ER entity should be specified. 
Chain-of-Custody Forms-The laboratory must present copies of all sample COC forms and Request for Analysis Forms, when applicable, for all samples received during the reporting period. This information is employed to ascertain difficulties or problems in communication or transfer of samples from the field to the laboratory. It is aiso used to trace the number of ER samples in the laboratory.

Out-of-Control Incidents-The laboratory must present all out-of-control incidents relating to ER samples, with copies of corrective action reports generated during the reporting period. These reports should be produced as a normal work activity in the laboratory QA process and be readily available for copying and inclusion in the MPR. The laboratory must specifically identify samples affected by project and field or client sample number, as appropriate.

The contents of these reports should be included in the Case Narratives. Examples of out-of-control incidents are as follows:

- Discrepancies noted during sample receipt and log-in [e.g., sample temperature, preservative $(\mathrm{pH})$ if applicable, and sample identification].

- Out-of-control points or conditions identified from control charts.

- Errors identified at the laboratory bench.

- Discrepancies noted during data review and reporting.

- Missed holding times.

\section{Changes to Laboratory-}

- QA and QC changes: The laboratory must describe and justify significant changes in its QAVQC protocol or program as it relates to ER samples.

- Method changes: The laboratory must identify new methods and/or changes to old methods which have been implemented.

- Personnel changes: The laboratory must identify changes in laboratory key personnel, including QA personnel. This will require submittal of resumes for new personnel.

- Facility changes: The laboratory must identify significant changes that have occurred in the facility within the reporting period. This must include changes in ownership, certifications (EPA, state, etc.), instrumentation, laboratory space, location, etc.

Response to MPR Comments-7he laboratory must respond in writing to previous MPR comments by the APO, through inclusion into the next MPR. These responses should reference the applicable MPR and identify the specific comment being addressed. 


\subsubsection{Scheduled Reviews}

All laboratories will be automatically scheduled to undergo reviews every 6 to 12 months, or at any time a problem arises. Conditions necessary for a scheduled review:

- The labriatory must currently be performing analyses for ER or

- The laboratory must be required for anticipated future work.

Scheduled reviews will follow the same basic pattern as initial reviews, with the addition of information from MPRs and past ER project work. The laboratory will be reviewed in five areas.

- Review of the current LQAP per Sect. 5.1.1.

- Submission of PE data per Sect. 5.1.2.

- Successful on-site laboratory audit per Sect. 5.1.3.

- Review of MPRs related to ER projects per Sect. 5.1.4.1.

- Review of past analyses submitted on ER projects.

\subsubsection{Performance Audits}

Several incidents may occur which require performance audits for all or part of a laboratory's operation. Conditions that may precipitate an additional laboratory review follow.

- Problems found in MPRs or in final data reports on projects.

- New methods used in the laboratory.

- Suspension of the laboratory by another review agency.

- A review is considered necessary by the ER project manager or the APO.

\subsection{OPERATIONAL REQUIREMENTS}

\subsection{Laboratory Organization and Personnel}

All changes in laboratory organization and personnel must be reported immediately to the APO, the ER project manager, and the engineering firm, if any, contracting the laboratory. Such changes may include operation mergers or acquisitions, expansions, relocation, management, and changes in $\mathrm{QA}$ or key technical personnel. Regulatory actions towards the organization such as suspension of contracts for EPA Special Analytical Services or Regular Analytical Services, as well as all legal actions against the laboratory must be reported immediately. 


\subsection{Standard Operating Procedures}

The laboratory must have written SOPs detailing each facet of work performed. These SOPs shall be reviewed and signed by the LQAC and must be available to personnel at the work station. Copies of regulatory procedures alone are not sufficient to meet this requirement; laboratory-specific means of implementation must be described. SOPs must be kept as controlled documents.

\subsection{Holding Time Requirements}

It must be clearly understood that three sets of regulations pertaining to holding times are in effect. The SW-846 and NPDES regulations state that holding times begin at the time of sample collection. The CLP states that holding times begin from Verified Time of Sample Receipt at the laboratory. When data validation is performed for any method (SW-846, NPDES, or CLP), the holding time begins on the date of sample collection. It is the policy of ER that holding times begin on the day of sample collection for all methods. Samples must be shipped by overnight delivery on the day of collection. Holding times to be used shall be so noted in the WP and shall be listed by analysis method, along with the type and volume of bottle used and storage conditions.

The ER policy regarding holding times is as follows:

- Holding times begin on the day of sample collection.

- Sample shipment and delivery must be coordinated with the laboratory to maintain sample integrity.

- Holding times must be identified in each project work plan or scope of work for each parameter or group of parameters to be analyzed.

- Holding times are measured in days from the date of sampling, unless the holding time is specified in hours or some other unit of time.

- Extraction holding times are met when the sample is placed into the appropriate medium.

- The time between completion of extraction and the beginning of concentration shall not exceed 1 day unless specified otherwise in a particular method.

- Postextraction or digestion, analytical holding times, begin when sample extraction or digestion is initiated.

- Holding time ends when the analysis, resulting in reported data, has been initiated (i.e., semivolatile GC/MS extract material is injected into the last instrumentation). If the final reported data results from a dilution or reinjection of the sample, this analysis must have been completed within the holding time.

- For organics, storage between the time of extraction and concentration shall be at $4^{\circ} \mathrm{C}$. Storage for metals following digestion may be at room temperature. 
- Medium- or high-concentration volatile organics shall not be held following extraction; their analysis must take place immediately after extraction. Volatile organics are to be analyzed by the low-level method unless the concentration criteria listed for medium- or high-concentration analysis in the requested method are met.

\subsection{Sample Receipt Requirements}

- The laboratory must sign air bills upon receipt and keep copies in the project file.

- Shipping container custody seals must be inspected and the condition documented.

- Integrity of the coolant or cooler temperature must be determined and documented.

- Condition of the samples must be documented.

- The pH of preserved samples (except volatile organics) must be checked upon receipt if specified in the WP and documented. The pH of volatile organics must be checked upon analysis and documented. Alternatively, the WP may specify that preservatives be checked and documented at time of sample collection and again at time of analysis. The check at time of collection must be with $\mathrm{pH}$ paper or a $\mathrm{pH}$ meter; addition of acid alone does not constitute a check.

- Any breakage, discrepancy, or improper preservation will be noted by the laboratory as an cut-of-control event and must be documented on an out-of-control form with the corrective action taken. The out-of-control form must be signed and dated by the custodian and any other person responsible for corrective action. The sample custodian must notify the contractor of discrepancies in shipments.

\subsection{Chain-of-Custody Requirements}

- All COC forms accompanying ER samples must be signed and dated upon receipt. The original COC should be the one sent with the samples unless the WP specifies otherwise.

- Copies of COCs accompanying ER samples must be maintained as part of the formal analysis data package.

- Copies of COCs accompanying ER samples must be included with the MPR.

- The laboratory must maintain an internal sample tracking system for all ER samples, regardless of the QC level associated with the analysis. Energy Systems does not require a written COC for transfers within a building; if the sample is sent to another building, COC documentation is required. For CLP work, EPA specifies that "the custody of EPA samples must be traceable from the time the samples are collected to the time they are introduced as evidence in legal procedures. The contractor shall have procedures ensuring that EPA sample custody is maintained and documented." (CLP SOWs, Exhibit F). For Resource Conservation and Recovery Act (RCRA) Facility Investigations, RCRA requires "Provision for a laboratory sample custody log consisting of serially numbered standard laboratory-tracking report sheets; and specification of laboratory sample custody procedures for sample handling, storage, and disbursement for analysis" (EPA 1989). Therefore, internal formal COC must be specified in the WP if the project warrants it. 


\subsubsection{Error Correction Requirements}

Any changes in entries in laboratory notebooks or on computer-printed data must be corrected by drawing a single line through the error and initialing and dating the new entry. The use of correction tape or fluid is not acceptable.

\subsubsection{Requirements for Cleanliness of Sample Containers}

In general, glass bottles with Teflon ${ }^{\text {me }}$ lids are used for organic samples, and polypropylene or polyethylene bottles are used for metals and other inorganics. The following specifies required bottle-cleaning procedures and documentation. If bottles are cleaned in the laboratory, bottle blanks must be performed on each cleaned lot of bottles, and verification of bottle cleanliness documented and records maintained. Bottle blanks are cleaned bottles (1\% of total) filled with laboratory water and the water analyzed for the analytes usually collected in that type of bottle. The method of analysis must be consistent with the method that will be used for the collected samples; for instance, if the 502.2 drinking water method will be used for volatile organic determination in groundwater samples, use of the CLP volatile organic analysis method for bottle blanks is not acceptable because of the difference in detection limits.

If precleaned bottles are purchased, this must be noted in the project WP or the LQAP. Certificates of analyses verifying bottle cleanliness must be kept on file, and the lot number must be recorded during sample collection.

All bottles should be capped, labeled, and packed in a cooler or box during shipment to the field. Bottles should be stored in a clean area.

\subsubsection{Cleaning Procedure for Glass Bottles (Except Volatile Organics)}

- Wash glass bottles, Teflon " liners, and caps in hot tap water with laboratory-grade nonphosphate detergent.

- Rinse three times with tap water.

- Rinse with 1:1 nitric acid (metals-grade), prepared with American Society for Testing and Materials (ASTM) Type II deionized water.

- Rinse three times with ASTM Type II deionized water.

- Rinse with pesticide-grade methylene chloride using $20 \mathrm{ml}$ for 0.5 -gal container and $5 \mathrm{ml}$ for 4-oz and 8-oz containers.

- Oven dry at $125^{\circ} \mathrm{C}$. Allow to cool to room temperature in an enclosed, clean area.

\subsection{Cleaning Procedure for Bottles Used for Volatile Organics}

- Wash glass vials, Teflon"-backed septa, Teflon " liners, and caps in hot tap water, using laboratory-grade nonphosphate detergent.

- Rinse three times with tap water. 
- Rinse three times with ASTM Type II deionized water.

- Oven dry vials, septa, and caps at a minimum of $125^{\circ} \mathrm{C}$.

- Allow vials, septa, and liners to cool to room temperature in an enclosed, clean environment.

- Seal 40-ml vials with septa (Teflon" side down) and cap.

- Store in a clean area.

\subsubsection{Cleaning Procedure for Plastic Bottles}

- Wash plastic bottles and caps in hot tap water with laboratory-grade nonphosphate detergent.

- Rinse with 1:1 nitric acid (metals-grade), prepared with ASTM Type II deionized water.

- Rinse three times with ASTM Type II deionized water.

- Invert and air dry in a clean area.

\subsubsection{Requirements for Reporting Out-of-Control Events}

ER requires that the APO be notified of all out-of-control events and that the corrective action be completed. Events that impede the analyses of ER samples must be reported to the ER project manager immediately. An example of this type of event would be the breakdown of a GC/MS system used for volatiles that could not be repaired for several days. If the laboratory cannot use another instrument in its facility, provisions shall be made for another ER-approved laboratory to analyze the samples.

Corrective action reports shall be signed by the laboratory director or the LQAC and shall discuss the following topics.

- When and where the out-of-control event occurred.

- Who discovered the out-of-control event, and who took corrective action.

- What analyses were being conducted. This must include a list of all samples affected. Sample problems and possible effects must be discussed.

- Corrective actions must be described, along with any measures enacted to prevent a recurrence of the problem.

- An explanation for the out-of-control event. A copy of subject control charts or other data describing the out-of-control conditions shall be included in the corrective action report.

All out-of-control event documentation and copies of the corrective action reports shall be: 
- placed in the laboratory archive record for the sample(s) in question,

- placed in the LQAC's file, and

- included in the MPR.

\subsubsection{Document Control Requirements}

ER requires that the laboratory maintain copies of all data packages, calibration records, and other quality-related records until the ER project manager either asks for the records or writes a letter requesting destruction of same. The laboratory must develop an SOP providing instructions for record keeping, document control, tracking, retrieval, and storage.

\subsection{Requirements for Traceability of Standards}

All standards available as such must be traceable by the National Institute of Standards and Testing (NIST) or EPA. The laboratory must maintain documentation of traceability. When NIST- or EPA-traceable standards are not available, documentation of the material used must still include the lot number, dilutions made, calculations, date of preparation, activity on date of testing, and other periinent information as applicable. The traceability requirement applies to all calibration standards and spike solutions. The laboratory must maintain clear, concise records for all working standards used in the laboratory.

\section{LABORATORY QUALTY CONTROL SAMPLES}

The types of laboratory QC samples are method blanks, LCSs (blank spikes), MSs, MSDs, and duplicates. These samples are defined in the following sections. Table 5.1 lists the percentage of laboratory QC samples per project QC level.

\subsubsection{Method Blank}

Method blanks are used to determine the existence and magnitude of possible contamination encountered during sample preparation. They consist of ASTM Type II water for water samples and clean sand or soil for soil samples. They are carried through the entire analytical procedure with the samples. At least one method blank must be prepared with each batch of samples. A batch cannot exceed 20 samples and is determined by the number of samples of similar matrix which can be processed simultaneously through the entire preparation process. 
42

Table 5.1 Laboratory quality control sample frequency

\begin{tabular}{|c|c|c|c|c|c|}
\hline Type of sample & Level A & Level B & Level C & Level D & Level E \\
\hline Method blank & $N / A^{a}$ & $1 / \mathrm{batch}^{b}$ & $1 /$ batch & 1/batch & 1/batch \\
\hline $\begin{array}{l}\text { Laboratory control } \\
\text { sample }\end{array}$ & N/A & Optional & $1 /$ batch & 1/batch & 1/batch \\
\hline MS/MSD_for organics & N/A & MS only & $1 / 20^{6}$ & $1 / 20$ & Optional \\
\hline $\begin{array}{l}\text { MS/Duplicate-for } \\
\text { inorganics }\end{array}$ & N/A & MS only & $1 / 20$ & $1 / 20$ & Optional \\
\hline Duplicates $^{d}$ & N/A & $1 / 20$ & $1 / 20$ & $1 / 20$ & Optional \\
\hline $\begin{array}{l}{ }^{a} \mathrm{~N} / \mathrm{A}=\text { not applicabl } \\
{ }^{b} \mathrm{~A} \text { batch cannot exce } \\
\text { 'If the analytical batct } \\
\text { included in the batch. } \\
{ }^{d} \text { Duplicates are perfo } \\
\text { for Level B. In Level } \mathrm{B} \text {, }\end{array}$ & $\begin{array}{l}\text { samples. } \\
\text { tains less }\end{array}$ & $\begin{array}{l}\text { MSDs } \\
\text { ired. }\end{array}$ & similar & MS/ $/$ & $\begin{array}{l}\text { ust be } \\
\text { led, except }\end{array}$ \\
\hline
\end{tabular}

\subsubsection{Laboratory Control Samples}

In Levels C, D, and E, an LCS shall be analyzed with each batch and recoveries plotted on control charts. The LCS consists of known concentrations of analytes representative of the contaminants to be determined, which are added to laboratory ASTM Type II water and carried through the entire preparation and analysis process. Commercially available LCSs or those from EPA may be used. LCS standards that are prepared in house must be made from a source independent of that of the calibration standards. In methods using surrogates, the method blank may be used as the LCS. Each surrogate compound must be plotted on control charts. For more information, see Sect. 5.4.

\subsubsection{Matrix Spikes and Matrix Spike Duplicates}

An MS is an aliquot of a sample spiked with known quantities of compounds and subjected to the entire analytical procedure. It is used to indicate the appropriateness of the method for the matrix by measuring recovery or accuracy. Accuracy is the nearness of a result or the mean of a set of results to the true or accepted value. An MSD is a second aliquot of the same sample as the MS with known quantities of compounds added. The purpose of the MSD, when compared to the MS, is to determine method precision. Precision is the measure of the reproducibility of a set of replicate results among themselves or the agreement among repeat observations made under the same conditions. MSs and MSDs are performed per 20 samples of similar matrix in QC Levels C and D. MSs and MSDs are optional for QC Level E. If the analytical batch contains less than 20 samples of similar matrix, MS and MSDs must be included in the analytical batch. 


\subsubsection{Duplicate Samples}

Duplicate samples are identical splits of individual samples which are analyzed by the laboratory to test for method reproducibility or precision. These samples are split in the laboratory during sample preparation. Duplicates are used mainly for inorganic analysis, and MSDs are used in organics. Duplicates are performed per 20 samples of similar matrix in QC Levels C and L. Duplicates are optional for QC Level E.

\subsection{LABORATORY CONTROL SAMPLE PROGRAM REQUIREMENTS}

The laboratory will employ a laboratory control sample program that monitors sample preparation and analysis. The LCS program is monitored through the use of control charts. The purpose of this program is to demonstrate that the laboratory process for : nple preparation and analysis is in control. The LCS program is required for all ER projects.

An LCS is a laboratory blank with a known amount of analyte(s) added or a commercially available standard consisting of known analyte concentrations representative of the contaminants to be determined. Analytes selected for spiking should be representative of the compound class. It is suggested that:

- For volatile organic compounds (VOCs) and semivolatile organic compounds, the method blank can be used as the LCS. All surrogates in the method blank must be monitored on control charts.

- For pesticides, at least two target compounds are added to a blank other than the method blank. These compounds are monitored on control charts.

- For polychlorinated biphenyls (PCBs), at least one target Aroclor is added to a blank other than the method blank. The compound is monitored on a control chart. If pesticides and PCBs are both being determined, it is recommended that a single LCS with both pesticide and PCB spikes be used.

- For metals determined by inductively coupled plasma (ICP), an LCS must be monitored by means of control charts for at least three metals.

- For metals determined by atomic absorption [graphite furnace atomic absorption (GFAA), furnace atomic absorption (FAA), and cold-vapor atomic absorption (CVAA)], an LCS must be monitored by means of a control chart for each element.

- For wet chemical methods, an appropriate LCS for each method must be used (e.g., for cyanide, a control standard of sodium cyanide from a source other than that used for calibration may be spiked into water and analyzed with the water samples). The value for the control is monitored on the control chart.

It is recommended that two types of matrix blanks be employed. One type of matrix is the laboratory blank water. The second type of matrix is a laboratory soil or blank sand. This soil can be pulverized and homogenized. If the soil used is known to contain some analytes of interest, no spiking may be required. The LCS matrix should be comparable to the sample matrix (i.e., analyze water control samples when water samples are analyzed). 
In the LCS, problems encountered with matrix effects and sample nonhomogeneity will be minimized through the use of blank water and well characterized soil. This information, used in conjunction with sample MS recoveries, can aid in determining whether an out-ofcontrol condition is because of laboratory problems or matrix problems.

\subsubsection{Control Charts}

Control charts provide a useful tool in assessing analytical performance through the graphic display of a parameter's variability over tinic. The parameter plotted on the chart is related to control sample testing either directly in terms of concentration or indirectly in terms of derived information [i.e., means, ranges, percent recoveries (\%Rs), relative percent differences (RPDs), or slopes of least-square data fits].

The laboratory must provide a brief description of the basic methodology for control chart use. Considerations covered include:

- establishing the number of LCSs per run sequence,

- determining parameters to be plotted against time,

- defining a statistical/mathematical basis for establishing and updating warning and control limits, and

- identifying shifts and trends that may typically be revealed by these charts.

\subsubsection{Administration of Control Charts}

The LQAP will address the following aspects of administering control charts.

- What types of laboratory activities the control charts will monitor.

- How often the LCSs will be run.

- How soon after results are obtained the charts will be monitored.

- Who is responsible for reading the charts.

- How changes in personnel, equipment, or processes will affect the charts.

- How often and under what circumstances limits will be updated.

- What corrective actions will be taken for out-of-control events.

\subsubsection{Statistical Approach to Control Charts}

Formulas used for the calculation of control chart limits must be provided. Conventional control chart formulas are based on normally distributed measurements and short-term variation. When data fit these conditions, the charts should perform as desired. Otherwise, the charts will either falsely signal out-of-control warnings more frequently than usual, fail to detect existing out-of-control conditions as often as they ordinarily would, or both (for 
different types of out-of-control situations). To correct any problems caused by improperly fitting control charts, the laboratory may propose alternate methods for setting control chart limits. All such proposals must include data and supportive statistical evidence. Possible alternate statistical approaches can include using nonparametric techniques, using medians instead of averages for the centerlines, identifying sources of variation, using long-term varation instead of short-term variation in setting limits, and transforming the data.

\subsubsection{Minimum Statistical Control Charting}

At a minimum, the laboratory must create an LCS control chart for each method of analysis and sample matrix. These charts will monitor laboratory measurements obtained from the LCS.

Each control chart must consist of a centerline, two warning limits, and two control limits. Control limits are calculated by moving range or by standard deviation.

If charting software does not mark the limits, the reviewer of the control chart needs to locate them to evaluate the data properly.

A minimum of 20 points per chart will be obtained before the initial attempt to establish control limits. If the laboratory does not have 20 points, recommended EPA recovery limits for the method must be used until the necessary 20 points are attained. Once control limits are established, they remain in effect until a change in the process warrants recalculation of control limits on a new set of at least 20 points. The laboratory would attempt to identify reasons for the process change and submit a corrective actions report explaining the reason for changing control limits. It is acceptable for a program to be used that recalculates control limits each time a point is entered or recalculates control limits every 20 points, but this must be noted in the monthly progress reports and the LQAP. Laboratory control limits shall be equal to or within EPA limits whenever such EPA limits exist. Any new points generated are to be plotted on the control chart within $24 \mathrm{~h}$.

\subsubsection{Criteria for an Out-of-Control Condition}

The laboratory must specify its criteria for defining an out-of-control condition related to control chart limits and patterns in the LQAP. A laboratory process for a particular analyte will be considered out of control whenever, at a minimum, any one of the following conditions is demonstrated by control chart monitoring of that analyte.

- Any one point is outside control limits.

- Any three consecutive points are outside warning limits.

- Any eight consecutive points are on the same side of the centerline.

- Any six consecutive points are such that each point is larger (or smaller) than its immediate predecessor.

- Any obvious cyclic pattern is seen in the points. 
If a software program is used that is not capable of flagging data that are outside these criteria, it is the responsibility of laboratory personnel to flag these out-of-control conditions manually.

\subsubsection{Reactions to Out-of-Control Conditions on Control Samples}

The LQAP must describe steps that will be taken in the event of an out-of-control condition. The laboratory must identify what action will be taken when warning and/or control limits are exceeded. Warning conditions may only require more frequent observations of a piece of equipment; control conditions require shutting down an instrument and implementing corrective action. Out-of-control events detected in the control charts must be documented in a corrective action report.

\subsection{ANALYTICAL METHOD REQUTREMENTS}

Following are analytical method requirements specific to ER projects.

- Only approved analytical methods such as those shown in Table 5.2 for water and soil are to be used for analysis of ER samples.

- All volatile and semivolatile organic analyses conducted by GC/MS must meet all quality control criteria set by the most current EPA CLP methods. When a non-CLP method such as SW-846 is required, the recommended QAVQC of the methods will be required by APO. This includes demonstration of required tuning and calibration of the GC/MS prior to analysis and every $12 \mathrm{~h}$; analysis of those blanks, surrogates, MS/MSDs and internal standards (IS) set by the method at the required frequency; and evaluation of that data in accordance with the limits established by the method. Compound identification; detection limit calculations and reporting of tentatively identified compounds (TIC) must also meet the requirements of the method. These requirements are mandatory regardless if the specified method is from SW-846 or the NPDES. TICs are not required for the TCLP analysis.

- For nitroaromatics, the appropriate method(s) developed by the U.S. Army Toxic and Hazardous Materials Agency or EPA shall be used.

- The Energy Systems Common Laboratory Practices cornmittee is developing a list of standardized protocols that have regulatory approval or for which regulatory approval can be sought. Methods that are not in Table 5.2 and unconventional methods or deviations that have not gone through the Common Laboratory Practices process must be indicated to the ER project manager. The project manager has several support sources to assist in evaluating the effectiveness and appropriateness of a method: the chemist on the project team, the APO, AESG, and the Common Laboratory Practices committee. 
Table 5.2 Analytical methods

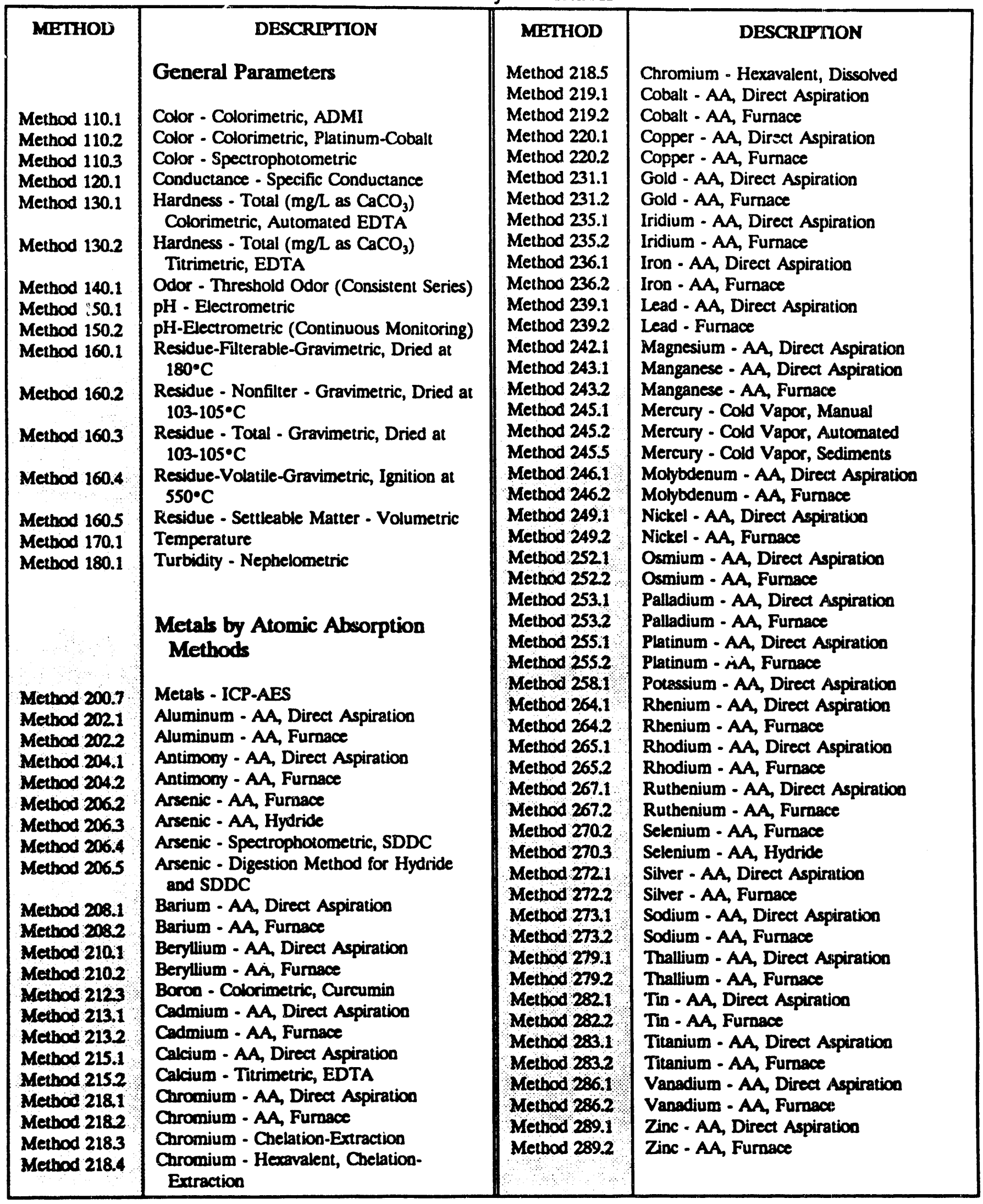


Table 5.2 (continued)

\begin{tabular}{|c|c|c|c|}
\hline METHOD & DESCRIPTION & METHOD & DESCRIPTION \\
\hline Method 300.0 & Anions - Ion Chromatography & $\begin{array}{l}\text { Method } 352.1 \\
\text { Method } 353.1\end{array}$ & $\begin{array}{l}\text { Nitrate - Colorimetric, Brucine } \\
\text { Nitrate-Nitrite - Colorimetric, Automated } \\
\text { Hydrazine Reduction }\end{array}$ \\
\hline Method 305.1 & Acidity - Titrimetric & Method 353.2 & Nitrate-Nitrite - Colorimetric, Automated \\
\hline Method 310.1 & Alkalinity - Titrimetric (pH 4.5) & & Cadmium Reduction \\
\hline Method 310.2 & $\begin{array}{l}\text { Alkalinity - Colorimetric, Automated } \\
\text { Method Orange }\end{array}$ & Method 353.3 & $\begin{array}{l}\text { Nitrate-Nitrite - Colorimetric, Manual } \\
\text { Cadmium Reduction }\end{array}$ \\
\hline Method 320.1 & Bromide - Titrimetric & Method 354.1 & Nitrite - Spectrophotometric \\
\hline Method 325.1 & $\begin{array}{l}\text { Chloride - Colorimetric, Automated } \\
\text { Ferricyanide, AA I }\end{array}$ & $\begin{array}{l}\text { Method } 360.1 \\
\text { Method } 360.2\end{array}$ & $\begin{array}{l}\text { Oxygen-Dissolved - Membrane Electrode } \\
\text { Oxygen-Dissolved-Modified Winkler: (Full }\end{array}$ \\
\hline Method 325.2 & $\begin{array}{l}\text { Chloride - Colorimetric, Automated } \\
\text { Ferricyanide, AA II }\end{array}$ & Method 365.1 & $\begin{array}{l}\text { Bottle Technique) } \\
\text { Phosphorus - All Forms - Colorimetric }\end{array}$ \\
\hline Method 325.3 & Chloride - Titrimetric, Mercuric Nitrate & & Automated, Ascorbic Acid \\
\hline Method 330.1 & $\begin{array}{l}\text { Chlorine - Total Residual - Titrimetric, } \\
\text { Amperometric }\end{array}$ & Method 365.2 & $\begin{array}{l}\text { Phosphorus - All Forms - Colorimetric, } \\
\text { Ascorbic Acid, Single Reagent }\end{array}$ \\
\hline Method 330.2 & $\begin{array}{l}\text { Chlorine- Total Residual- Titrimetric, Back- } \\
\text { Iodometric }\end{array}$ & Method 365.3 & $\begin{array}{l}\text { Phosphorus - All Forms - Colorimetric, } \\
\text { Ascorbic Acid, Two Reagents }\end{array}$ \\
\hline Method 330,3 & $\begin{array}{l}\text { Chlorine - Total Residual - Titrimetric, } \\
\text { Iodometric }\end{array}$ & Nethod & $\begin{array}{l}\text { Phosphorus - Total - Colorimetric, } \\
\text { Automated, Block Digestor, AAII }\end{array}$ \\
\hline Method 330.4 & $\begin{array}{l}\text { Chlorine-Total Residual-Titrimetric, DPD- } \\
\text { FAS }\end{array}$ & $\begin{array}{l}\text { Metbod } 370.1 \\
\text { Method } 375.1\end{array}$ & $\begin{array}{l}\text { Silica - Dissolved - Colorimetric } \\
\text { Sulfate - Colorimetric, Automated }\end{array}$ \\
\hline Method 330.5 & $\begin{array}{l}\text { Chlorine - Total Residual - } \\
\text { Spectrophotometric, DPD }\end{array}$ & Method 375.2 & $\begin{array}{l}\text { Chloranilate } \\
\text { Sulfate - Colorimetric, Automated Methyl }\end{array}$ \\
\hline Meth & Cyanide - Amenable to Chlorination & & Thymol Blue, - AAII \\
\hline Method 335.2 & $\begin{array}{l}\text { Cyanide Total - Titrimetric, } \\
\text { Spectrophotometric }\end{array}$ & $\begin{array}{l}\text { Method } 375.3 \\
\text { Method } 375.4\end{array}$ & $\begin{array}{l}\text { Sulfate - Gravimetric } \\
\text { Sulfate - Turbidimetric }\end{array}$ \\
\hline Method 335.3 & $\begin{array}{l}\text { Cyanide Total - Colorimetric, Automated } \\
\text { UV }\end{array}$ & $\begin{array}{l}\text { Method } 376.1 \\
\text { Method } 376.2\end{array}$ & $\begin{array}{l}\text { Sulfide - Titrimetric, iodine } \\
\text { Sulfide - Colorimetric, Methylene Blue }\end{array}$ \\
\hline Method 340.1 & $\begin{array}{l}\text { Fluoride - Colorimetric, SPADNS with } \\
\text { Bellack Distillation }\end{array}$ & $\begin{array}{l}\text { Method } 377.1 \\
\text { Method } 405.1\end{array}$ & $\begin{array}{l}\text { Sulfite - Titrimetric } \\
\text { Biochemical Oxygen Demand (BOD) }\end{array}$ \\
\hline Method 340.2 & $\begin{array}{l}\text { Fluoride - Potentiometric, Ion Selective } \\
\text { Electrode }\end{array}$ & Method 410.1 & $\begin{array}{l}\left(5 \mathrm{~d}, 20^{\circ} \mathrm{C}\right) \\
\text { Chemical Oxygen Demand - Titrimetric, }\end{array}$ \\
\hline Method 340.3 & $\begin{array}{l}\text { Fluoride - Colorimetric, Automated } \\
\text { Complexone } \\
\text { Iodide - Titrimetric }\end{array}$ & Method 410.2 & $\begin{array}{l}\text { Mid-Level } \\
\text { Chemical Oxygen Demand - Titrimetric, } \\
\text { Low-Level }\end{array}$ \\
\hline $\begin{array}{l}\text { Method } 343.1 \\
\text { Method } 350.1\end{array}$ & $\begin{array}{l}\text { Nitrogen - Ammonia - Colorimetric, } \\
\text { Automated Phenate }\end{array}$ & Method 410.3 & $\begin{array}{l}\text { Chemical Oxygen Demand - Titrimetric, } \\
\text { High-Level for Saline Waters }\end{array}$ \\
\hline Method 350.2 & $\begin{array}{l}\text { Nitrogen - Ammonia - Colorimetric, } \\
\text { Titrimetric, Potentiometric, Distillation } \\
\text { Procedure }\end{array}$ & $\begin{array}{l}\text { Method } 410.4 \\
\text { Method } 413.1\end{array}$ & $\begin{array}{l}\text { Chemical Oxygen Demand-Colorimetric, } \\
\text { Automated; Manual } \\
\text { Oil and Grease - Gravimetric, Separatory }\end{array}$ \\
\hline Method 350.3 & $\begin{array}{l}\text { Nitrogen - Ammonia - Potentiometric, Ion } \\
\text { Selective Electrode }\end{array}$ & Method 4132 & $\begin{array}{l}\text { Funnel Extraction, Total Recoverable } \\
\text { Oil and Grease - Spectrophotometric, }\end{array}$ \\
\hline Method 351.1 & $\begin{array}{l}\text { Kjeldahl-Total - Colorimetric, Automated } \\
\text { Phenate }\end{array}$ & Method 415.1 & $\begin{array}{l}\text { Infrared, Total Recoverable } \\
\text { Organic Carbon-Combustion or Oxidation, }\end{array}$ \\
\hline Method 351.2 & $\begin{array}{l}\text { Kjeldahl-Total - Colorimetric, Semi- } \\
\text { Automated Block Digester - AAII }\end{array}$ & Method 415.2 & $\begin{array}{l}\text { Total } \\
\text { Organic Carbon-UV Promoted, Persulfate }\end{array}$ \\
\hline Method 351.3 & $\begin{array}{l}\text { Kjeldahl-Total - Colorimetric - Titrimetric, } \\
\text { Potentiometric }\end{array}$ & & $\begin{array}{l}\text { Oxidation, Total } \\
\text { Petroleum - Spectrophotometric, Infrared, }\end{array}$ \\
\hline & $\begin{array}{l}\text { Kjeldahl-Total - Potentiometric - Ion } \\
\text { Selective Electrode }\end{array}$ & & Hydrocarbons, Total, Recoverable \\
\hline
\end{tabular}


Table 5.2 (continued)

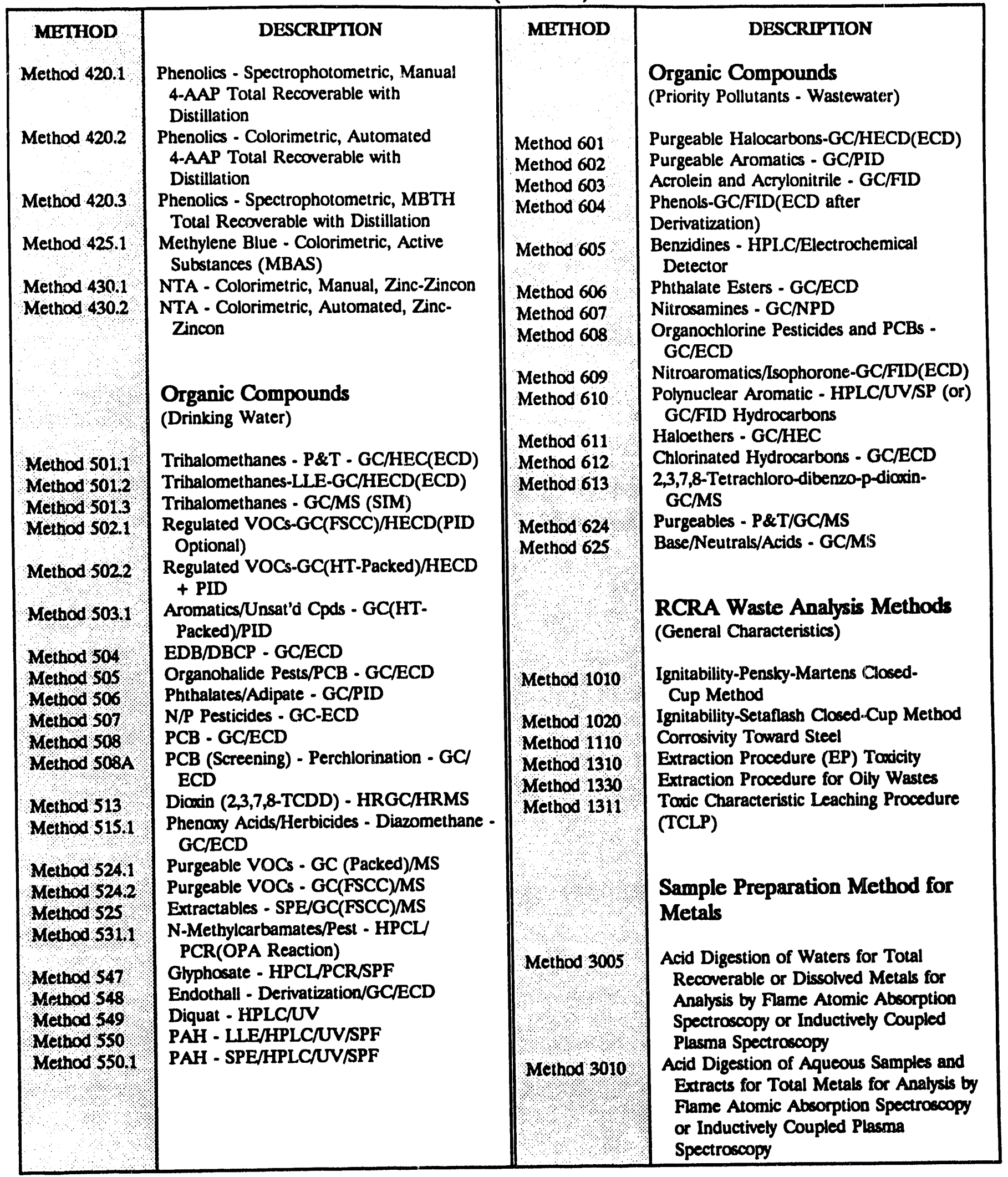


Table 5.2 (continued)

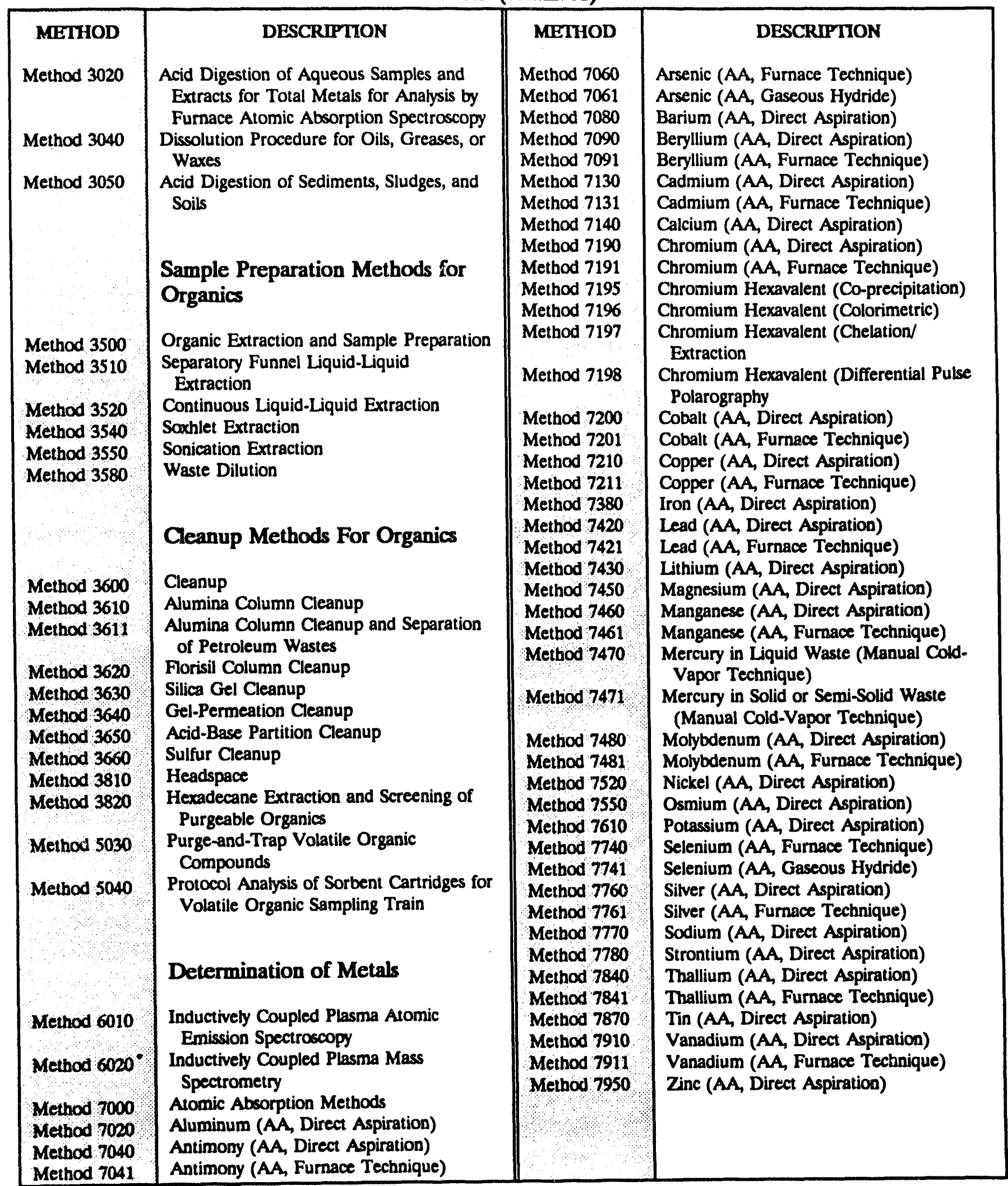


Table 5.2 (continued)

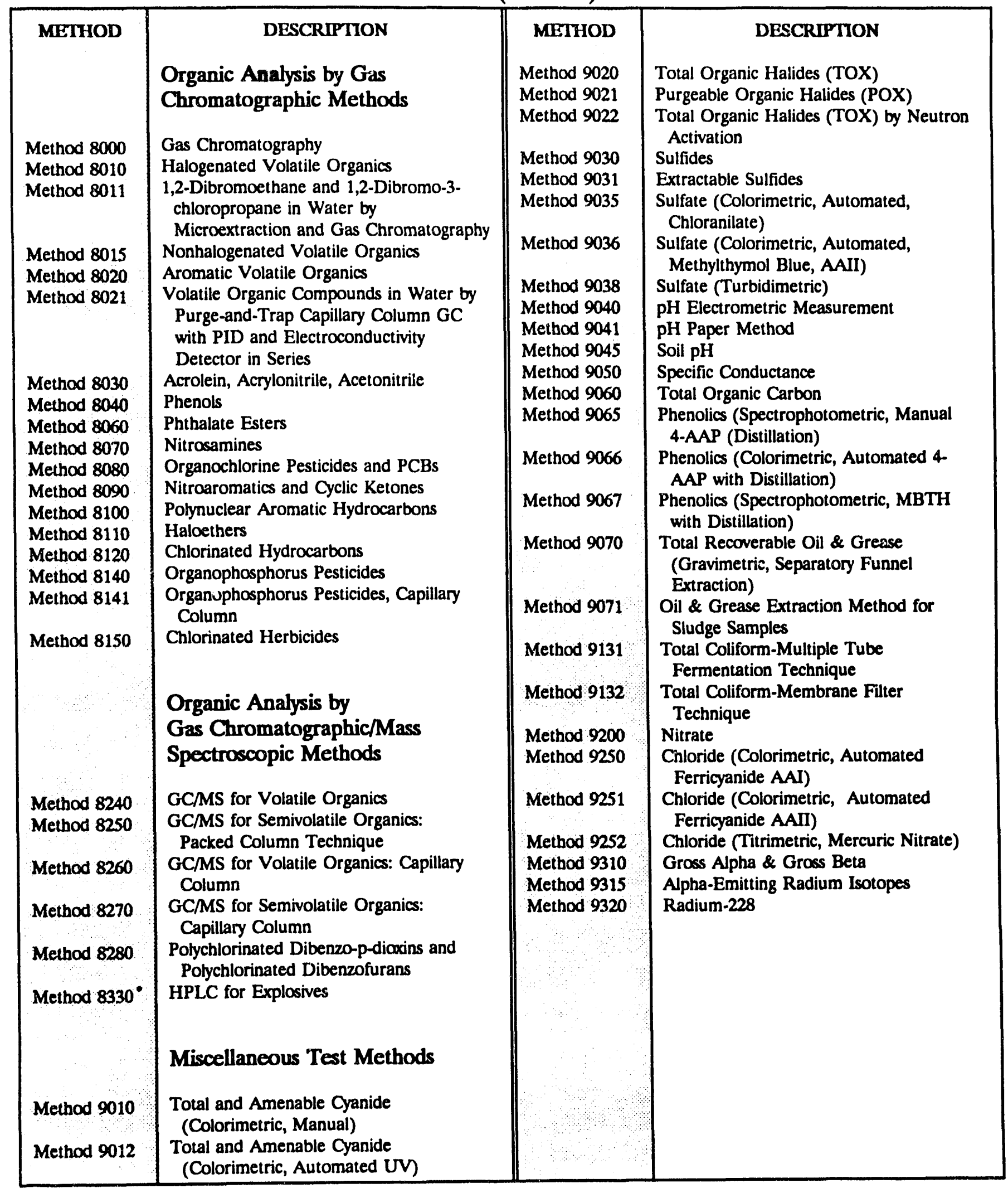

Draft 
Unconventional methods submitted for approval must demonstrate laboratory capability to handle the specific matrix, identify the parameters of interest, and achieve the required detection limits. As a minimum, the method must state:

- Sample handling

- Sample preparation

- Instrumentation and operating conditions

- Analysis steps, including all relevant technical information

- Applicable QC measures and criteria

If the method is approved, the project manager incorporates it into the WP. Regulatory approval of the WP will then include approval of the method or deviation for that project. Unconventional analytical methods for work that was ongoing before the distribution of these requirements generally will have received project approval by this course. The method must receive approval from the project manager before implementation by the laboratory.

Available protocols for analysis of radiochemical, air, and biota samples may be less standardized or less detailed than those for other sample types. Typically, sample preparation and QC call for more input on the part of the laboratory. Methods are available from the references below, but laboratory SOPs must be carefully reviewed to ensure that they provide results consistent with the DQOs, as described for unconventional methods.

\section{Biota}

"Residues of Organochlorine Pesticides and Polychlorinated Biphenyls: Autopsy Data for Bald Eagles." J. Pestic. Monit. 9:11-14. (Cromartie, D. W., et al. 1975).

\section{Radiochemistry}

Eastern Environmental Radiation Facility Radiochemistry Procedures Manual. EPA 520/ 5-84-006. (EPA 1984).

\section{Air}

National Institute of Occupational Safety and Health. NIOSH Manual of Analytical Methods, Publication No. 84-100, U.S. Department of Health and Human Services, Cincinnati, Ohio, February (NIOSH 1984).

SW-846, Method 0010, Appendix B. Total Chromatographable Organic Material Analysis.

Emission Assessments of Conventional Siationasy Combustion Systems: Methods and Procedures Manual for Sampling and Analysis, Interagency Energy/Environmental R\&D Program, Industrial Environmental Laboratory Research Laboratory, RTP. EPA-600/7-79-029a (EPA 1979). 
- An LCS shall be analyzed with each batch and recoveries plotted on control charts (refer to Sect. 5.4).

- Optimum batch size is determined by the number of samples of similar matrix with the ability to be processed simultaneously through the entire preparation and analytical process within a normal work shift. For example, if 5 samples can be extracted but 20 can be analyzed by the instrument during a normal shift, the batch size is 5 samples.

- For all GC quantitative methods (not screening), second-column confirmation shall be performed on all positive responses for the analytes of interest. Second-column confirmation must be performed with the same types of QC samples as were required for the quantitation column.

- When performing petroleum hydrocarbon, oil and grease, anion (such as nitrates, sulfates, and chloride), and other wet chemical methods, an MS and duplicate are required for every 20 samples of similar matrix. Similar matrix is defined as either soil or water from the same site.

- All methods require calibration.

- For all semivolatile and volatile analysis by GC/MS, the current calibration protocol of the method shall be used. The current criteria shall be used for frequency of calibration and for checking the system performance calibration compounds and calibration check compounds (CCCs).

- For other methods, a minimum of three different concentration standards plus a blank standard for each analyte shall be analyzed for initial calibration. The initial calibration curve shall be plotted and the correlation coefficient and response factors evaluated. The laboratory must indicate the acceptance criteria to be used for the initial calibration curve. Calibration shall include one standard of a concentration at method detection limits. If samples are not within calibration range, appropriate dilution shall be performed to bring samples into range.

- All GC analysis must use the external calibration method.

- An MS and MSD are required for organic analyses for every 20 samples of similar matrix. For metals analysis, a duplicate and an MS are required for every 20 samples of similar matrix.

- Postdigestion spikes are required for furnace analysis and when the MS analysis is outside acceptable limits. 


\section{REQUIREMENTS FOR SAMPLE ANALYSIS DELIVERABLES REQUIREMENTS}

Specific deliverables have been designated for each QC level. The sample deliverables are designed to ensure that all necessary data for the successful completion of the project is obtained. All laboratories receiving ER samples are required to provide these deliverables.

\subsection{LEVEL A}

A formal final report is not required; the only deliverables are sample results. The daily single-point calibration must be kept on file. More information on Level A deliverables can be found in Requirements for Quality Control of Field Activities, ES/ER/TM-11 (Energy Systems to be issued in 1993).

\subsection{LEVEL B}

Deliverables include sample results, method blanks, three-point calibration, and continuing calibration checks. More information on Level B deliverables can be found in Requirements for Quality Control of Field Activities, ES/ER/TM-11 (Energy Systems to be issued in 1993).

\subsection{LEVEL C}

Submission of the forms referred to in Table 6.1, or similar forms, is required. The CLP forms indicated in Table 6.1 are based on the current CLP SOW; the forms required for the organics analysis (OLM01.8) series and inorganics analysis (ILM02.1) series of CLP SOWs are listed in Table 6.1. If forms other than CLP forms are used, the laboratory must include a copy of those forms in the LQAP or send them to the ER project manager for approval before initiating work. Raw data are not required; however, the laboratory must define its policy of raw data retention.

For investigative projects, buth hard copy and electronic data transmission (EDT) will be required once the EDT system is defined. Iniernal laboratories may be exempted from submitting QC data for certain monitoring projects if the information is readily retrievable and the project manager specifies the exemption in the WP. The Common Laboratory Practices Committee will define the EDT system. Data should be retrievable and sortable. QC information has to be the same electronically as on hard copy. Error-free transmission has to be ensured. QC requirements for EDT are being investigated and will be incorporated into future revisions of this document.

\subsection{LEVEL D}

The main difference between Level $D$ deliverables and Level $C$ deliverables is that Level D deliverables include raw data. A CLP, or CLP-equivalent, package is required for Level D, regardless of the analytical method. It is also a requirement that all LCS control 
charts associated with the ER project be supplied with the data package. Electronic deliverables consist of all the data on the forms.

\subsection{LEVEL E}

The minimum information to be submitted must include laboratory case narratives, $\mathrm{COC}$, sample results, method blank data, initial and continuing calibration data, and control charts from the LCS data. Exact deliverables will be stated in the WP and approved by the ER project manager before initiating work.

Table 6.1. Data set deliverables for Level $\mathbf{C}$ quality assurancenb

\begin{tabular}{ll}
\hline Method requirements & Deliverables \\
\hline
\end{tabular}

Requirements for all methods:

- Holding time information and methods requested

Signed chain-of-custody forms

... Discussion of laboratory analysis, including any laboratory problems

Case narratives

- LCS with results on control charts. Run with each batch of samples processed

Control charts

Organics: GC/MS analysis

- Sample results, including TICs

CLP Form 1 or equivalent

- Surrogate recoveries

CLP Form 2 or equivalent

- Matrix spike/spike duplicate data

- Method blank data

CLP Form 3 or equivalent

CLP Form 4 or equivalent

- GC/MS tune

CLP Form 5 or equivalent

- GC/MS initial calibration data

CLP Form 6 or equivalent

- GC/MS continuing calibration data

CLP Form 7 or equivalent

- GCMS internal standard area data

CLP Form 8 or equivalent

GC analysis

- Sample results

- Surrogate recoveries

- Matrix spike/spike duplicate

CLP Form 1 or equivalent

CLP Form 2 or equivalent

CLP Form 3 or equivalent

- Method blank data

CLP Form 4 or equivalent

- Initial calibration data of single

For VI PEST-1 and PEST-2 component analytes 
Table 6.1 (continued)

\begin{tabular}{|c|c|c|}
\hline \multicolumn{2}{|r|}{ Method requirements } & Deliverables \\
\hline- & $\begin{array}{l}\text { Initial calibration for multicomponent } \\
\text { analytes }\end{array}$ & Form VI PEST-3 \\
\hline- & Analyte resolution summary & Form VI PEST-4 \\
\hline- & Calibration verification summary & Form VII PEST-1 \\
\hline- & Calibration verification summary & Form VII PEST-2 \\
\hline- & Analytical sequence & Form VIII PEST \\
\hline- & Florisil cartridge check & Form IX PEST-1 \\
\hline - & Pesticide GPC calibration & Form IX PEST-2 \\
\hline- & $\begin{array}{l}\text { Pesticide identification summary for } \\
\text { single component analytes }\end{array}$ & Form X PEST-1 \\
\hline- & $\begin{array}{l}\text { Pesticide identification summary for } \\
\text { multicomponent analytes }\end{array}$ & Form X PEST-2 \\
\hline & If calibration factors are used & $\begin{array}{l}\text { A form listing each analyte, the } \\
\text { concentration of each standard, the } \\
\text { relative calibration factor, the mean } \\
\text { calibration factor, and the \%RSD }\end{array}$ \\
\hline & If a calibration curve is used & $\begin{array}{l}\text { Calibration curve and correlation } \\
\text { coefficient }\end{array}$ \\
\hline- & Continuing calibration data & CLP Form 9 or equivalent \\
\hline- & Positive identification & CLP Form 10 or equivalent \\
\hline \multicolumn{3}{|l|}{ Metals } \\
\hline - & Sample results & CLP Form 1 or equivalent \\
\hline- & Initial and continuing calibration & $\begin{array}{l}\text { CLP Form } 2 \text { or equivalent, and } \\
\text { dates of analyses and calibration } \\
\text { curve, and the correlation coefficient } \\
\text { factor }\end{array}$ \\
\hline- & Method blank & $\begin{array}{l}\text { CLP Form } 3 \text { or equivalent and dates } \\
\text { of analyses }\end{array}$ \\
\hline - & ICP interference check sample & $\begin{array}{l}\text { CLP Form } 4 \text { or equivalent and dates } \\
\text { of analyses }\end{array}$ \\
\hline - & Spike sample recovery & CLP Form 5A or equivalent \\
\hline - & $\begin{array}{l}\text { Postdigestion spike sample recovery for } \\
\text { ICP metals }\end{array}$ & CLP Form 5B or equivalent \\
\hline- & Postdigestion spike for GFAA & CLP Form SB or equivalent \\
\hline - & Duplicates & CLP Form 6 or equivalent \\
\hline- & LCS & CLP Form 7 or equivalent \\
\hline- & Standard addition & $\begin{array}{l}\text { CLP Form } 8 \text { or equivalent and dates } \\
\text { of analyses }\end{array}$ \\
\hline
\end{tabular}




\begin{tabular}{cll}
\hline \multicolumn{1}{c}{ Method requirements } & \multicolumn{1}{c}{ Deliverables } \\
\hline - & Preparation log & CLP Form 13 or equivalent \\
- & Run log & CLP Form 14 or equivalent \\
Wet chemistry & \\
- Sample results & Report result \\
- Matrix spike duplicate & Percent recovery (\%R) and relative \\
- Method blank & percent difference (RPD) \\
- Initial calibration & Report result \\
- Continuing calibration check & Calibration curve and correlation \\
- $\quad$ Run log & coefficient \\
Radiochemical analysis & \%R and percent difference (\%D) \\
- Sample results & Copy of run log \\
- Initial calibration & \\
- Efficiency check & Report results \\
- Background determinations & Efficiency determination \\
- Minimum detectable activity & \%D from calibration \\
- Method blank & Report results \\
- Spike recovery results & Report results \\
- Internal standard results & Report results \\
- Duplicate results & Spike added and \%R \\
- Self absorption factors (alpha and beta) & Standard added and \%R \\
- Run log & Report results and RPD \\
\hline
\end{tabular}

a Contract Laboratory Program (CLP) forms are based on the organic analysis (OLM01.8) and inorganic analysis (ILM 02.1) series of CLP SOWs. (EPA 1988d)

${ }^{b}$ Forms must be submitted electronically, as hard copy, or both ways depending on the specifications in the Work Plan. 


\section{DATA VALIDATION GUIDELINES}

Data must be validated against acceptable criteria to provide assurance that data are adequate for the intended use. The project must indicate in the QAPP the process and criteria to be used to validate project data, as well as the percentage of data to be validated. The validation process compares the objective vs the actual through evaluation of the SPARCC parameters. The process will consist of data editing, screening, checking, auditing, verification, flagging, and review. A qualified representative, designated by the ER project manager or the contractor but independent of the laboratory, will perform data validation. The laboratory will not perform data validation; validation is independent of laboratory data review. The ER project manager or the contractor will certify in writing that data have been validated and flagged in accordance with the defined process.

When data validation is performed, the holding time begins at the date of sample collection for all methods. Samples must be shipped by overnight delivery on the day of collection. Holding times to be used shall be so noted in the WP and shall be listed by analysis method along with the type of bottle used, preservation requirements, and storage conditions.

The ER policy regarding holding times is as follows:

- Holding times begin on the day of sample collection.

- Sample shipment and delivery must be coordinated with the laboratory to maintain sample integrity.

- Holding times must be identified in each project work plan or scope of work for each parameter or group of parameters to be analyzed.

- Holding times are measured in days from the date of sampling, unless the holding time is specified in hours or some other unit of time.

- Extraction holing times are met when the sample is placed into the appropriate medium.

- The time between completion of extraction and the beginning of concentration shall not exceed one day unless specified otherwise in a particular method.

- Postextraction or digestion, analytical holding times, begin when sample extraction or digestion is initiated.

- Holding time ends when the analysis, resulting in reported data, has been initiated (i.e., semivolatile GC/MS extract material is injected into the last instrumentation). If the final reported data results from a dilution or re-injection or the sample, this analysis must have been completed within the holding time.

- For organics, storage between the time of extraction and concentration shall be at $4^{\circ} \mathrm{C}$. Storage for metals following digestion may be at room temperature.

- Medium- or high-concentration volatile organics shall not be held following extraction; their analysis must take place immediately after extraction. Volatile organics are to be 
analyzed by the low-level method unless the concentration criteria listed for medium- or high-concentration analysis in the requested method are met.

Volatile organics are to be analyzed by the low-level method unless the concentration criteria listed for medium- or high-concentration analysis in the requested meihod are met.

Tables 7.1 through 7.7 outline holding times, containers, and preservatives for some methods applicable to the ER Program. Methods not included in these tables will follow the holding times and preservation techniques stated by the method. In the ER Program, CLP holding times are consistent with CLP validation guidelines, and water samples for volatile organics must be preserved. Sample preservation is generally limited to $\mathrm{pH}$ control, chemical addition, refrigeration, and freezing.

It must also be noted that ER has specific requirements concerning blanks when performing data validation. The following guidelines regarding blanks must be followed:

- Blank results are not to be subtracted from sample values for any reason.

- Blank criteria apply to all blanks (e.g., field, equipment, trip, method). Where more than one blank is associated with a given group of samples, the evaluation shall be based on comperison with the associated blank having the highest concentration of contaminant.

ER project managers need to plan data validation as part of the project, plan for cost, obtain appropriate expertise to perform data validation, use the correct region/state requirements, and write validation procedures as part of the project plan. It needs to be noted that each region and/or state has differences in validation procedures, based on the Superfund procedures, Laboratory Data Validation Functional Guidelines for Evaluating Organics Analyses (EPA 1988c), and Laboratory Data Validation Functional Guidelines for Evaluating Inorganics Analyses (EPA 1988b).

The percentage of validation that needs to be performed is based on the DQOs of the project. The cost of validation is dependent on the level of validation required but should not exceed 10 to $15 \%$ of the analytical costs of the data to be validated. As electronic deliverables become more established, electronic compliance screening and some proportion of validation review can be expected.

\subsection{LEVEL A DATA VALIDATION GUIDEILNES}

Level A data require no data validation because only sample results are presented.

\section{LEVEL B DATA VALIDATION GUIDELINES}

Level B data do not undergo a validation process but must undergo a formal review process. The ER project manager or the engineering entity, if applicable, in concert with field laboratory analysts, will indicate in the QAPP the process and criteria to be used to review data. Acceptable criteria for data review must be defined prior to sample analysis. The process will address data editing, screening, and verification. Data verification must include checking calibration and blanks to ensure criteria have been met. The review process must include 
instructions for flagging samples associated with blanks or calibrations that are out of criteria. The engineering entity or its contractor shall use MS information to evaluate data.

The ER project manager or the contractor must certify in writing that data have been reviewed in accordance with the defined process.

\subsection{LEVEL C DATA VALIDATION GUIDELINES}

Listed below are the minimum validation criteria that will be used in evaluating analytical data for a Level C QC analysis. Validation procedures and criteria must be identified in the project WP, outlining validation of holding times, initial calibration, continuing calibration, blank vs sample results, and any project-specific considerations. The validation procedure for methods not listed here must be approved by the project manager and the APO, or their support designees. 


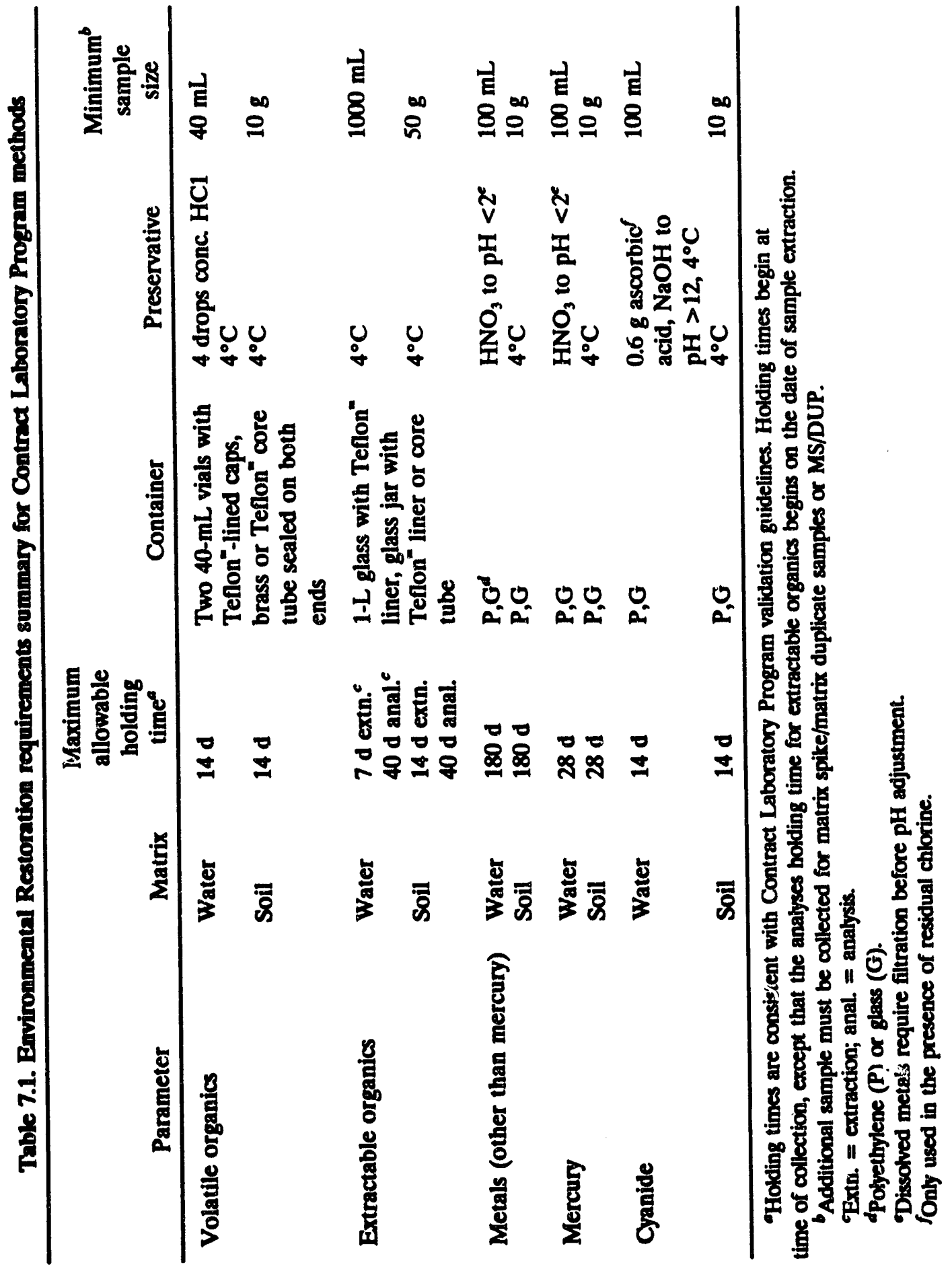




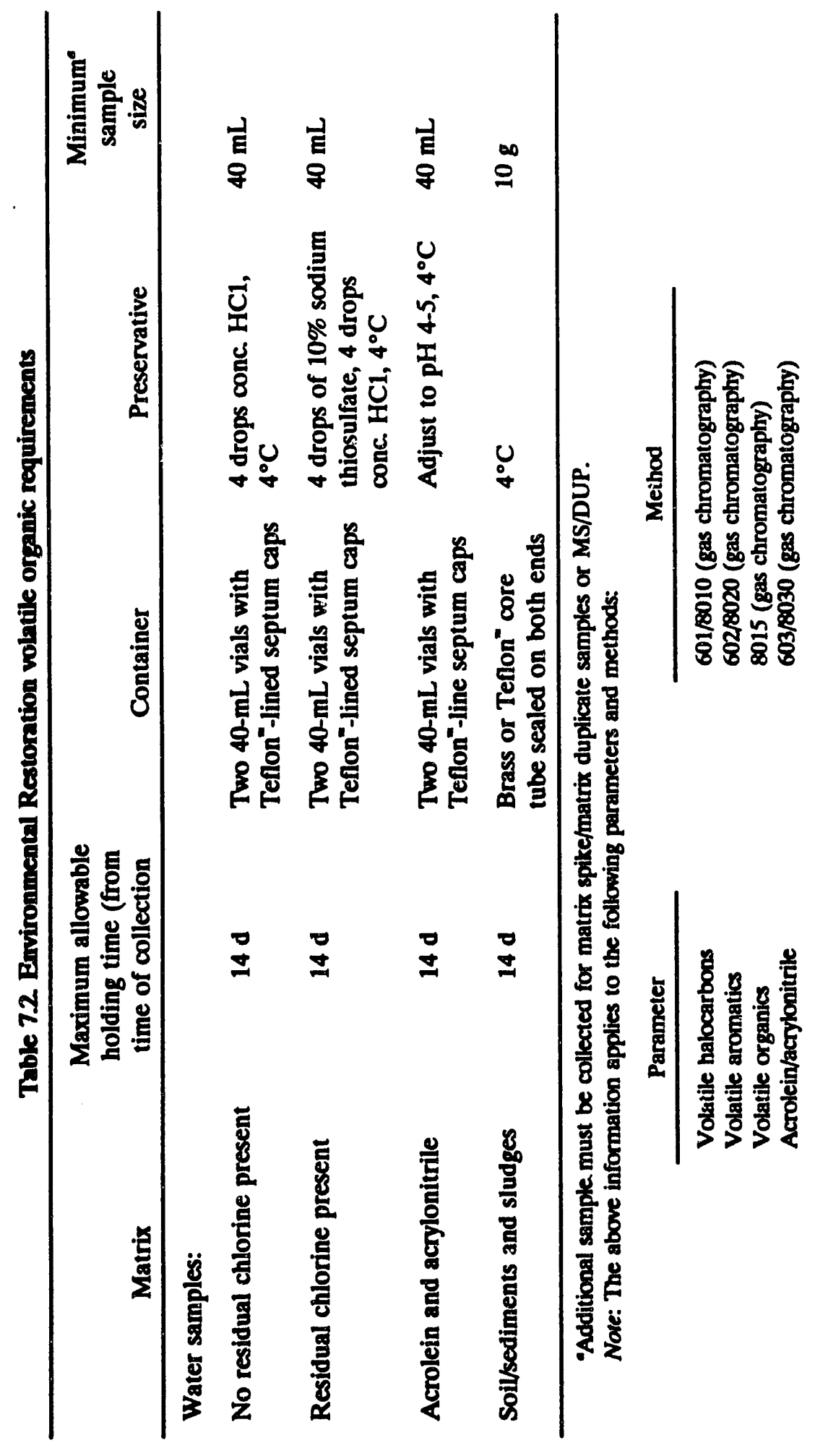




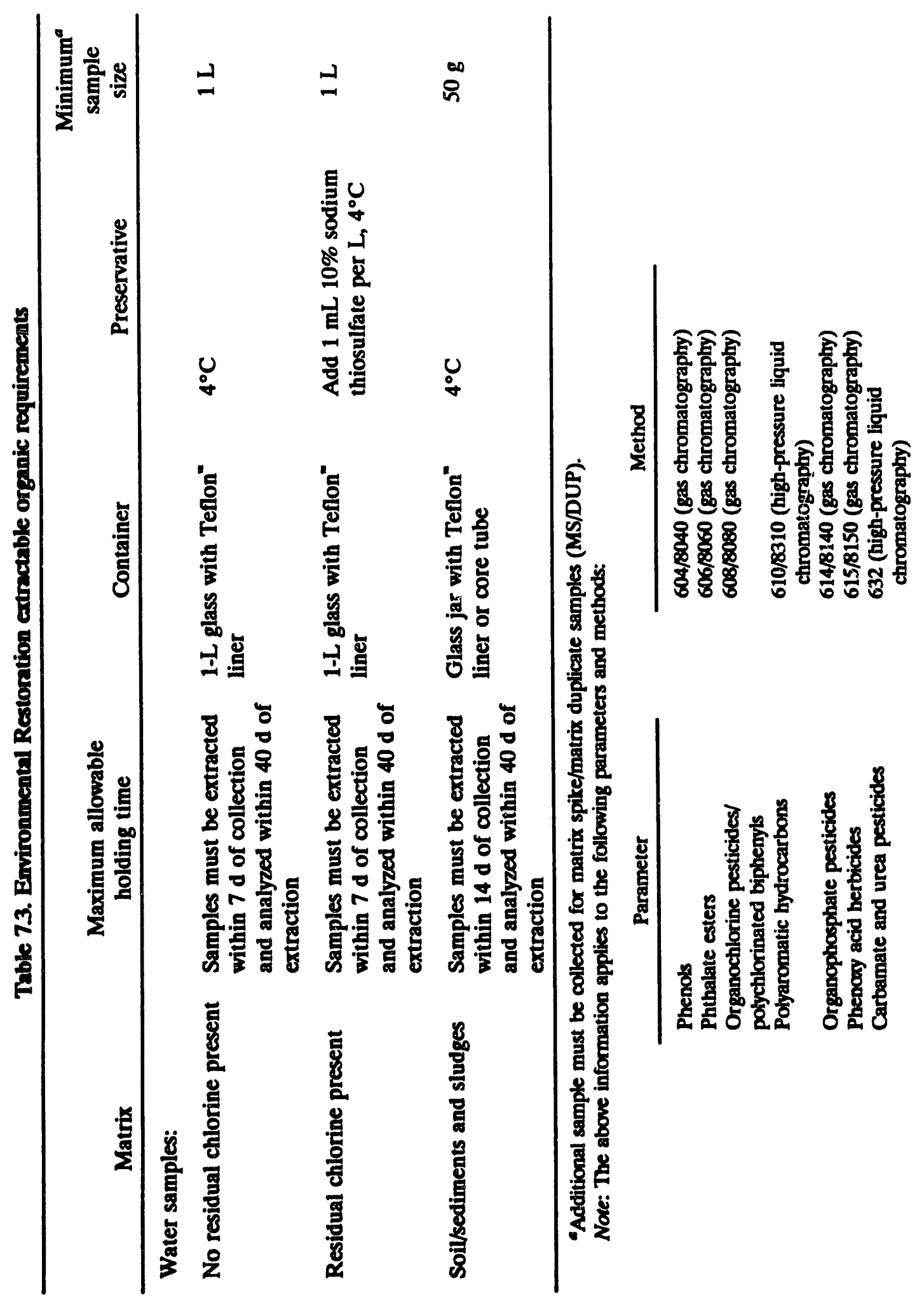




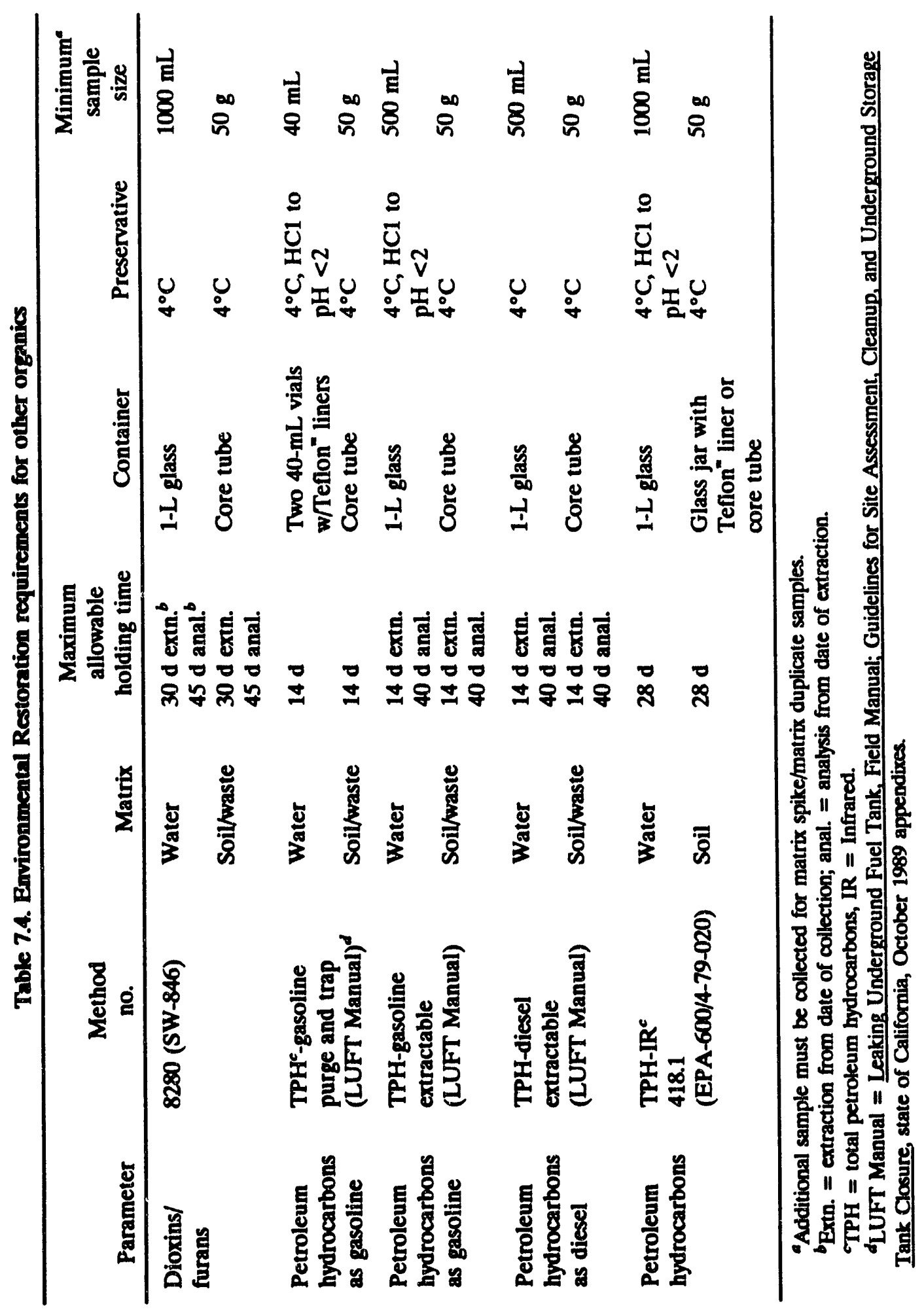




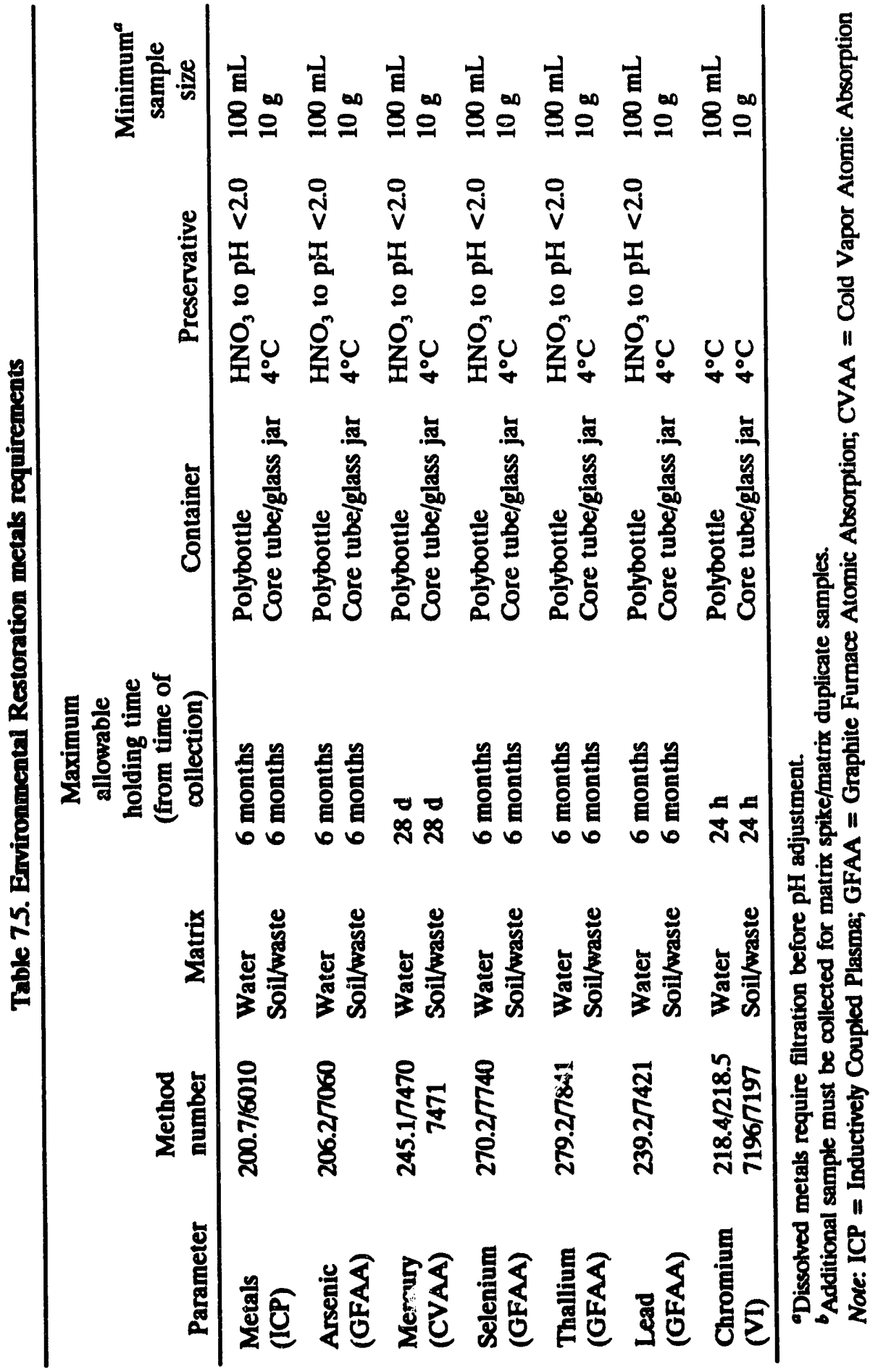




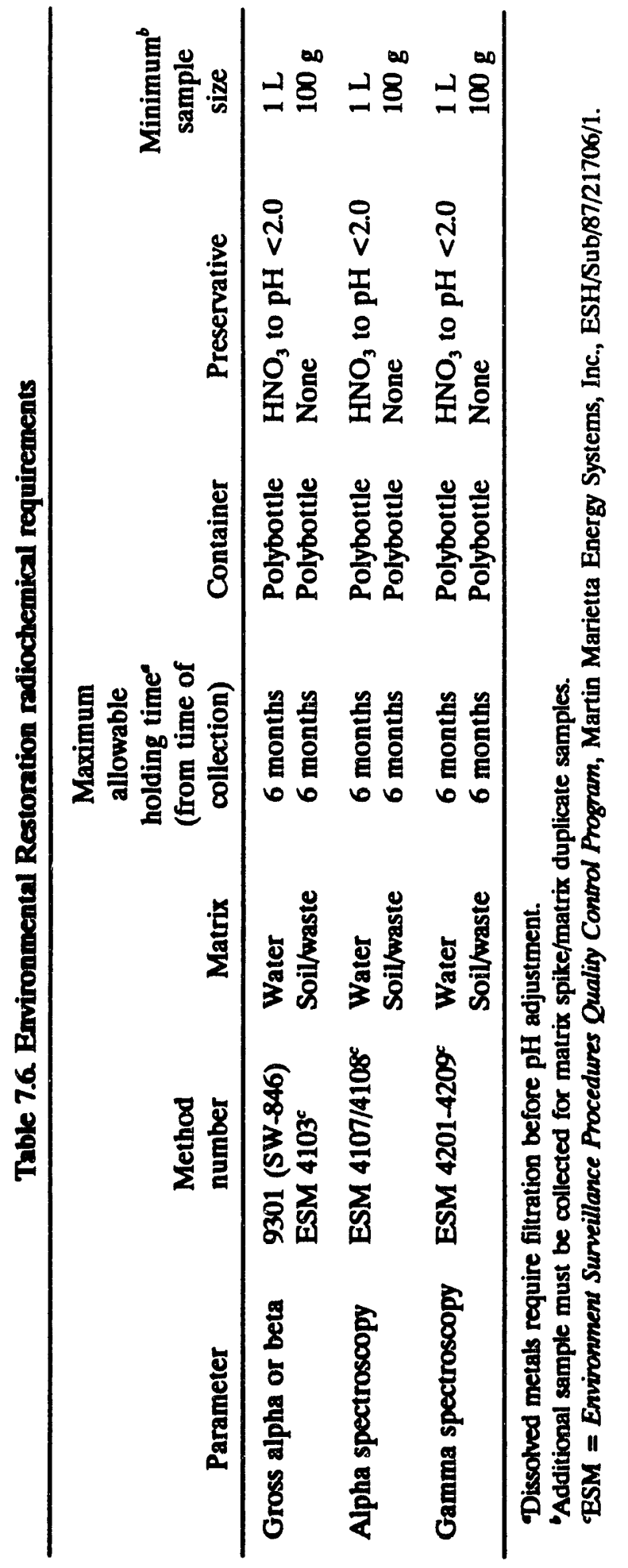


Table 7.7. Environmental Restoration requirements for general chemical analyses

\begin{tabular}{|c|c|c|c|c|c|}
\hline Parameter & $\begin{array}{l}\text { Method } \\
\text { number }\end{array}$ & Matrix & $\begin{array}{l}\text { Holding time } \\
\text { (from date of } \\
\text { collection) }\end{array}$ & Container & Preservative \\
\hline \multicolumn{6}{|l|}{100 Physical Properties } \\
\hline Color & $110.1, .2, .3$ & water & 48 hours & $P, G^{a}$ & $\mathrm{CoOl}, 4^{\circ} \mathrm{C}$ \\
\hline Conductance & 120.1 & water & 28 days & $\mathbf{P}, \mathbf{G}$ & $\mathrm{CoOl}, 4^{\circ} \mathrm{C}$ \\
\hline Hardness & $130.1, .2$ & water & 6 months & $\mathbf{P}, \mathbf{G}$ & $\mathrm{HNO}_{3}$ to $\mathrm{pH}<2$ \\
\hline $\mathrm{pH}$ & 150.1 & water & $\begin{array}{l}\text { Analyze } \\
\text { immediately }\end{array}$ & $\mathbf{P}, \mathbf{G}$ & None Req. \\
\hline Residue & $160.1, .2, .3, .4$ & water & 7 days & $\mathbf{P}, \mathbf{G}$ & $\mathrm{Cool}, 4^{\circ} \mathrm{C}$ \\
\hline Settleable Matter & 160.5 & water & 48 hours & $\mathbf{P}, \mathbf{G}$ & $\mathrm{Cool}, 4^{\circ} \mathrm{C}$ \\
\hline Temperature & 170.1 & water & $\begin{array}{l}\text { Analyze } \\
\text { immediately }\end{array}$ & P, G & None required \\
\hline Turbidity & 180.1 & water & 48 hours & $\mathbf{P}, \mathbf{G}$ & Cool, $4^{\circ} \mathrm{C}$ \\
\hline \multicolumn{6}{|l|}{200 Metals $^{b}$} \\
\hline Chromium VI & $218.4, .5$ & water & 24 hours & $\mathbf{P}, \mathbf{G}$ & $\mathrm{CoOl}, 4^{\circ} \mathrm{C}$ \\
\hline \multicolumn{6}{|l|}{$\begin{array}{l}300 \text { Inorganics, } \\
\text { Non-Metallics }\end{array}$} \\
\hline Acidity & 305.1 & water & 14 days & $\mathbf{P}, \mathbf{G}$ & $\mathrm{CoOl}, 4^{\circ} \mathrm{C}$ \\
\hline Alkalinity & $310.1, .2$ & water & 14 days & $\mathbf{P}, \mathbf{G}$ & $\mathrm{Cool}, 4^{\circ} \mathrm{C}$ \\
\hline Bromide & 320.1 & water & 28 days & $\mathbf{P}, \mathbf{G}$ & None required \\
\hline Chloride & $325.1, .2, .3$ & water & 28 days & $\mathbf{P}, \mathbf{G}$ & None required \\
\hline Chlorine & $\begin{array}{l}330.1, .2, .3 \\
.4, .5\end{array}$ & water & $\begin{array}{l}\text { Analyze } \\
\text { immediately }\end{array}$ & $\mathbf{P}, \mathbf{G}$ & None required \\
\hline Cyanides & $335.1, .2, .3$ & water & 14 days & $\mathbf{P}, \mathbf{G}$ & $\begin{array}{l}\mathrm{CoOl}, 4^{\circ} \mathrm{C} \\
\mathrm{NaOH} \text { to } \\
\mathrm{pH}>12 \\
0.6 \mathrm{~g} \text { ascorbic acid }\end{array}$ \\
\hline Fluoride & $340.1, .2, .3$ & water & 28 days & $\mathbf{P}, \mathbf{G}$ & None required \\
\hline Iodide & 345.1 & water & 24 hours & $\mathbf{P}, \mathbf{G}$ & $\mathrm{Cool}, 4^{\circ} \mathrm{C}$ \\
\hline \multicolumn{6}{|l|}{ Nitrogen } \\
\hline Ammonia & $350.1, .2, .3$ & water & 28 days & $\mathbf{P}, \mathbf{G}$ & $\begin{array}{l}\mathrm{CoOl}, 4^{\circ} \mathrm{C} \\
\mathrm{H}_{2} \mathrm{SO}_{4} \text { to } \mathrm{pH}<2\end{array}$ \\
\hline Kjeldahl, total & $351.1, .2, .3, .4$ & water & 28 days & $\mathbf{P}, \mathbf{G}$ & $\begin{array}{l}\mathrm{CoOl}, 4^{\circ} \mathrm{C} \\
\mathrm{H}_{2} \mathrm{SO}_{4} \text { to } \mathrm{pH}<2\end{array}$ \\
\hline Nitrate plus Nitrite & $353.1, .2, .3$ & water & 28 days & $\mathbf{P}, \mathbf{G}$ & $\begin{array}{l}\mathrm{CoOl}, 4^{\circ} \mathrm{C} \\
\mathrm{H}_{2} \mathrm{SO}_{4} \text { to } \mathrm{pH}<2\end{array}$ \\
\hline Nitrate & 352.1 & water & 48 hours & $\mathbf{P}, \mathbf{G}$ & $\mathrm{CoOl}, 4^{\circ} \mathrm{C}$ \\
\hline Nitrite & 354.1 & water & 48 hours & $\mathbf{P}, \mathbf{G}$ & $\mathrm{CoOl}, 4^{\bullet} \mathrm{C}$ \\
\hline $\begin{array}{l}\text { Dissolved corygen } \\
\text { probe }\end{array}$ & 360.1 & water & $\begin{array}{l}\text { Analyze } \\
\text { immediately }\end{array}$ & $\begin{array}{l}\text { G bottle } \\
\text { and top }\end{array}$ & None required \\
\hline WinkJer & 360.2 & water & 8 hours & $\begin{array}{l}G \text { bottle } \\
\text { and top }\end{array}$ & $\begin{array}{l}\text { Fix on site and } \\
\text { store in dark }\end{array}$ \\
\hline
\end{tabular}


Table 7.7 (continued)

\begin{tabular}{|c|c|c|c|c|c|}
\hline Parameter & $\begin{array}{l}\text { Method } \\
\text { number }\end{array}$ & Matrix & $\begin{array}{l}\text { Holding time } \\
\text { (from date of } \\
\text { collection) }\end{array}$ & Container & Preservative \\
\hline $\begin{array}{l}\text { Phosphorus } \\
\text { orthophosphate, } \\
\text { dissolved }\end{array}$ & $365.1, .2, .3$ & water & 48 hours & P, G & $\begin{array}{l}\text { Filter on site } \\
\mathrm{Cool}, 4^{\circ} \mathrm{C}\end{array}$ \\
\hline Hydrolyzable & $365.1, .2, .3$ & water & 28 days & $\mathbf{P}, \mathbf{G}$ & $\begin{array}{l}\mathrm{CoOl}, 4^{\circ} \mathrm{C} \\
\mathrm{H}_{2} \mathrm{SO}_{4} \text { to } \mathrm{pH}<2\end{array}$ \\
\hline Total & $365.1, .2, .3, .4$ & water & 28 days & $P, G$ & $\begin{array}{l}\mathrm{CoOl}, 4^{\circ} \mathrm{C} \\
\mathrm{H}_{2} \mathrm{SO}_{4} \text { to } \mathrm{pH}<2\end{array}$ \\
\hline $\begin{array}{l}\text { Total } \\
\text { dissolved }\end{array}$ & $365.1, .2, .3$ & water & 24 hours & P, G & $\begin{array}{l}\text { Filter on site } \\
\mathrm{CoOl}, 4^{\circ} \mathrm{C} \\
\mathrm{H}_{2} \mathrm{SO}_{4} \text { to } \mathrm{pH}<2\end{array}$ \\
\hline Silica & 370.1 & water & 28 days & P only & $\mathrm{Cool}, 4^{\circ} \mathrm{C}$ \\
\hline Sulfate & $375.1, .2, .3, .4$ & water & 28 days & P, G & $\mathrm{CoOl}, 4^{\circ} \mathrm{C}$ \\
\hline Sulfide & $376.1, .2$ & water & 7 days & P, G & $\begin{array}{l}\mathrm{Cool}, 4^{\bullet} \mathrm{C} \text { add } 2 \\
\text { ml zinc } \\
\text { acetate plus } \\
\mathrm{NaOH} \text { to } \mathrm{pH}>9\end{array}$ \\
\hline Sulfite & 377.1 & water & $\begin{array}{l}\text { Analyze } \\
\text { immediately }\end{array}$ & P, G & None required \\
\hline
\end{tabular}

${ }^{a}$ Polyethylene $(P)$ or glass $(G)$.

${ }^{b}$ Dissolved metals require filtration before $\mathrm{pH}$ adjustment.

\subsubsection{Petroleum Hydrocarbons (EPA Method 418.1)}

Holding Times-Ensure that holding times were met. Holding times are 28 days from the date of coliection. All samples must be properly preserved.

If the holding time was exceeded or samples were not preserved:

- Document that samples were not properly preserved.

- Document that holding times were exceeded.

- Flag all associated positive results as estimated (J).

- Flag all associated sample quantitation limits as estimated (UJ).

Note: The holding time of 28 days applies only to petroleum hydrocarbons analyzed by EPA Method 418.1. Holding times for other petroleum hydrocarbon analytical methods (including California Modified 8015) can be found in Table 7.4.

Initial Calibration-Ensure, at a minimum, that a three-point standard curve bracketing sample concentration is performed daily. The correlation coefficient must meet or exceed 0.995 before the analysis of samples.

If the minimum number of standards was not used for initial calibration: 
- Qualify data as unusable (R).

If the instrument was not calibrated daily before sample analysis:

- Qualify data as unusable (R).

If the correlation coefficient is less than 0.995:

- Qualify sample results greater than or equal to the Instrument Detection Limit (IDL) as estimated $(\mathrm{J})$.

- Qualify sample results less than the IDLs as unusable (R).

Continuing Calibration-Ensure that the continuing calibration falls within $\pm 20 \%$ of the midrange standard.

If the criterion is not met:

- Qualify sample results greater thari or equal to the IDL as estimated (J).

- Qualify sample results less than the IDL as unusable (R).

Blanks-Ensure that all associated blanks were analyzed.

If the concentration in the sample is less than or equal to five times the concentration found in the blank:

- The result is considered as a nondetect and flagged as such (U).

If the concentration in the sample is greater than five times the concentration found in the blank:

- The result is considered positive and no flag is required.

Laboratory Control Samples-Ensure that each sample is analyzed in a batch in which an LCS has been performed. Any LCS exceeding internal QC limits set by the laboratory for a given sample matrix shall require all data from the associated batch of samples to be closely inspected.

If no analytical problems are found:

- Data analyzed with the out-of-control point shall be discussed.

If problems are found in the analytical data:

- Ensure that samples associated with the batch were re-analyzed and data from re-analysis reported.

If holding times are exceeded in the re-analysis, both sets of data shall be presented. 
If the LCS results are outside internal laboratory limits and if the MS results are outside the laboratory limits, the data shall be qualified as follows:

- Qualify nondetects for all samples associated with the LCS as unusable (R).

- Qualify positive results for all samples associated with the LCS as estimated (J).

Matrix Spike/Matrix Spike Duplicate-Ensure that an MS/MSD has been associated with each sample. Control limits must be established by the laboratory. These criteria cannot be used alone to evaluate precision and accuracy. Flagging is not required.

\subsubsection{Gas Chromatograph/Mass Spectrometer Volatile Organics}

Validation for GC/MS volatile organics will essentially follow the CLP Functional Validation Guidelines. The criteria below are based on the CLP SOW, Revision OLM01.8.

Holding Times-Ensure that samples have been analyzed within 14 days from date of collection for water samples that are preserved and refrigerated. The same holding times are applied to soil samples.

If water samples are unpreserved, the holding time is seven days from date collected. If there is no indication of preservation, assume samples are unpreserved.

If the holding time was exceeded or samples were not preserved:

- Document that samples were not properly preserved.

- Document that holding times were exceeded.

- Flag all associated positive results as estimated (J).

- Flag all associated sample quantitation limits as estimated (UJ).

Gas Chromatograph/Mass SpectrometerTuning -Ensure that a bromofluorobenzene tune, meeting the CLP criteria, is completed every $12 \mathrm{~h}$ of sample analysis and that each sample is associated with a tune.

If tunes do not meet the expanded criteria as listed below:

$\mathrm{m} / \mathrm{z} \quad$ Ion abundance criteria

$50 \quad 8.0$ to $40.0 \%$ of mass 95

$75 \quad 30.0$ to $66.0 \%$ of mass 95

95 base peak, $100 \%$ relative abundance

965.0 to $9.0 \%$ of mass 95 (see note)

173 less than $2 \%$ of mass 174

$174 \quad 50$ to $120 \%$ of mass 95

$175 \quad 5.0$ to $9.0 \%$ of $\mathrm{m} / \mathrm{z} 174$

$176 \quad 93$ to $101 \%$ of $\mathrm{m} / \mathrm{z} 174$

$177 \quad 5.0$ to $9.0 \%$ of $\mathrm{m} / \mathrm{z} 176$ 
Note: All ion abundances must be normalized to the $\mathrm{m} / \mathrm{z} 95$, the nominal base peak, even though the ion abundance of $\mathrm{m} / \mathrm{z} 174$ be up to $120 \%$ that of $\mathrm{m} / \mathrm{z} 95$.

- Flag associated data as unusable (R).

Initial Calibration-The response factor of the compounds listed in Table 7.8 must meet the minimum RRF criteria at each concentration level and maximum \%RSD criteria for the initial calibration with allowances made for up to two volatile compounds. However, the RRFs for those two compounds must be greater than or equal to 0.010 , and the \%RSD of those two compounds must be less than or equal to $40 \%$ for the initial calibration to be acceptable. However, these compounds will still be qualified per appropriate data validation guidelines.

Table 7.8. Minimum relative response factors for initial calibration of volatile organics-CLP SOW Revision OLM01.8

\begin{tabular}{lc}
\hline Volatile compound & $\begin{array}{c}\text { Minimum } \\
\text { RRF }\end{array}$ \\
\hline Bromomethane & 0.100 \\
Vinyl chloride & 0.100 \\
1,1-Dichloroethene & 0.100 \\
1,1-Dichloroethane & 0.200 \\
Chloroform & 0.200 \\
1,2-Dichloroethane & 0.100 \\
1,1,1-Trichloroethane & 0.100 \\
Carbon Tetrachloride & 0.100 \\
Bromodichloromethane & 0.200 \\
cis-1,3-Dichloropropene & 0.200 \\
Trichloroethene & 0.300 \\
Dibromochloromethane & 0.100 \\
1,1,2-Trichloreoethane & 0.100 \\
Benzene & 0.500 \\
trans-1,3-Dichloropropene & 0.100 \\
Bromoform & 0.100 \\
Tetrachloroethene & 0.200 \\
1,1,2,2-Tetrachlorethane & 0.500 \\
Toluene & 0.400 \\
Chlorobenzene & 0.500 \\
Ethylbenzene & 0.100 \\
Styrene & 0.300 \\
Xylenes (total) & 0.300 \\
Bromofluorobenzene & 0.200 \\
\hline & \\
\hline
\end{tabular}


The following compounds (see Table 7.9) have no maximum \% RSD, or maximum percent criteria; however, these compounds must meet a minimum RRF criterion of 0.010 .

Table 7.9. Compounds that must meet a minimum RRF criterion of 0.010

\begin{tabular}{l||l}
\hline Acetone & 1,2-Dichloropropane \\
2-Butanone & 2-Hexanone \\
Carbon disulfide & Methylene chloride \\
Chloroethane & 4-Methyl-2-pentanone \\
Chloromethane & Toluene-d8 \\
1,2-Dichloroethene (total) & 1,2-Dichloroethane-d4 \\
\hline
\end{tabular}

If any compound listed in Table 7.8 has an RRF less than the required minimum criteria:

- Flag all positive results for that compound as estimated (J).

- Flag nondetects for that compound as unusable (R).

If any compound listed in Table 7.8 exceeds $20.5 \% \mathrm{RSD}$ :

- Flag positive results for that compound as estimated (J).

- Qualify nondetects using professional judgment.

Continuing Calibration-Ensure that the RRF for all compounds is greater than or equal to those listed in Tables 7.8 and 7.9 and all percentages are less than or equal to $20.5 \%$ RSD.

If any compound has an RRF of less than those listed in Tables 7.8 and 7.9:

- Flag positive results for that compound as estimated (J).

- Flag nondetects for that compound as unusable (R).

If any compound has a $\% \mathrm{D}$ greater than $25 \%$ :

- Flag positive results for that compound as estimated (J).

- Flag nondetects using professional judgment.

Blank-Ensure that all associated blanks were analyzed.

If a compound is found in a blank but not found in the associated sample, no action is taken.

If a compound is found in a blank and the associated sample the following two rules (5x and 10x) apply: 
- The 10x Rule applies to the four common laboratory contaminants listed below.

methylene chloride

acetone

toluene

2-butanone (methyl ethyl ketone)

- When the concentration of that compound is greater than or equal to the contractrequired quantitation limit (CRQL) but less than 10 times the highest concentration found in any blank, consider the result as a nondetect and flag it with a (U).

- When the concentration of that compound is less than the CRQL and less than 10 times the highest concentration found in any blank, report the result as the CRQL with a $(U)$ qualifier.

- When the concentration of the compound is greater than or equal to 10 times the highest concentration found in any blank, the result is considered as positive, and no flag is required.

- The 5x Rule applies to all compounds other than the four common laboratory contaminants listed previously.

- When the concentration of that compound is greater than or equal to the CRQL but less than 5 times the highest concentration found in any blank, consider the result as a nondetect and flag it with a (U).

- When the concentration of that compound is less than the CRQL and less than 5 times the highest concentration found in any blank, report the result as the CRQL with a (U) qualifier.

- When the concentration of the compound is greater than or equal to 5 times the highest concentration found in any blank, consider the result as positive, and no flag is required.

- Sample analytes not detected or detected at levels less than CRQL are reported as the CRQL with a (U) flag added.

If gross contamination exists:

- Flag all compounds affected as unusable (R).

If inordinate amounts of other compounds and/or TICs are found in any blank:

- Note this in the validation comments.

Laboratory Control Samples-Ensure that each sample is analyzed in a batch in which an LCS has been performed. Any LCS exceeding internal QC limits set by the laboratory for a given sample matrix shall require all data from the associated batch of samples to be closely inspected. 
If no analytical problems are found:

- Data analyzed with the out-of-control point shall be discussed.

If problems are found in the analytical data:

- Ensure that samples associated with the batch were re-analyzed and data from the reanalysis reported.

If holding times are exceeded in the re-analysis, both sets of data shall be presented.

If the LCS results are outside internal laboratory limits and if MS results are outside the CLP limits, the data shall be qualified as follows:

- Qualify nondetects for all samples associated with the LCS as unusable (R).

- Qualify positive results for all samples associated with the LCS as estimated (J).

Surrogates-Ensure that all surrogates are within CLP limits.

If any surrogate recovery is out of specification but greater than $10 \%$ recovery:

- Qualify positive results as estimated (J).

- Qualify nondetects with the CRQL as estimated (UJ).

If any surrogate shows less than $10 \%$ recovery:

- Flag positive results as estimated (J).

- Flag nondetects as unusable (R).

If any blank has surrogates out of specification:

- Flag results using professional judgement.

Matrix Spike/Matrix Spike Duplicate-Ensure that an MS/MSD has been associated with each sample. Control limits must be established by the laboratory for each spiking compound. These criteria cannot be used alone to evaluate precision and accuracy. Flagging is not required.

Internal Standard Area Performance-Ensure that IS area counts do not vary by more than a factor of $2(-50 \%$ to $+100 \%)$ from the associated calibration standard.

Retention time of the IS must not vary more than \pm 30 seconds from the associated calibration standard.

If an IS area count is outside $-50 \%$ or $+100 \%$ of the associated calibration standard:

- Flag positive results for compounds quantitated using that IS as estimated (J). 
- Flag nondetects for compounds quantitased using that IS with the CRQL as estimated (UJ).

If extremely low area counts are reported $c$ if performance exhibits a major, abrupt drop-off, a severe loss of sensitivity is indicated:

- Qualify nondetects as unusable (R). A discussion must be included in the case narrative describing the problem.

\subsubsection{Gas Chromatograph Volatile Organics}

Holding Times-Ensure that holding times were met and samples were properly preserved. Water samples that are preserved must be analyzed within 14 days. If water samples are unpreserved, the holding time is 7 days. If there is no indication of preservation, assume samples are unpreserved. Soil samples must be analyzed with 14 days.

If holding time was exceeded or samples were not preserved:

- Document that samples were not properly preserved.

- Document that holding times were exceeded.

- If the holding time is grossly excceded, use best professional judgment as to data reliability. The reviewer may flag all associated nondetect data as unusable (R).

- Flag all positive results as estimated (J).

- Flag all associated sample quantitation limits as estimated (UJ).

Calibration-Ensure that an external calibration procedure is used for quantitation by the laboratory.

If calibration factors are used for sample quantitation:

- For initial calibration, all \%RSD must be less than or equal to $20 \%$.

- For continuing calibration, all \%D must be less than $15 \%$.

If linear regression is used for sample quantitation:

- Verification of the calibration curve is required, and the correlation coefficient must be greater than or equal to 0.995 .

In the primary analysis, all standards are analyzed at the beginning of the $12-\mathrm{h}$ period, followed by tb : proper sample/standard sequence. Confirmation analysis requires a midlevel standard at the beginring of the $12-\mathrm{h}$ period. The midlevel standard must be repeated after every 10 samples.

If the criteria for initial calibration are not met:

- Flag all associated quantitative results as estimated (J). 
If the criteria for continuing calibration are not met:

- In the primary analysis, flag all associated quantitative results as estimated (J).

- In the confirmation analysis, use professional judgment as to data reliability.

If proper standards have not been analyzed:

- Use professional judgment as to data reliability.

Blanks-Ensure that all associated blanks were analyzed.

If a compound is found in a blank but not found in the associated sample, no action is taken.

If a compound is found in a blank and the associated sample, the following two rules ( $5 x$ and 10x) apply:

- The 10x Rule applies to the four common laboratory contaminants listed previously.

- When the concentration of that compound is greater than or equal to the CRQL but less than 10 times the highest concentration found in any blank, consider the result as a nondetect and flag it with a (U).

- When the concentration of that compound is less than the CRQL and less than 10 times the highest concentration found in any blank, report the result as the CRQL with a (U) qualifier.

- When the concentration of che compound is greater than or equal to 10 times the highest concentration found in any blank, consider the result as positive, and no flag is required.

- The 5x Rule applies to all compounds other than the four common laboratory contaminants listed previously.

- When the concentration of that compound is greater than or equal to the CRQL but less than 5 times the highest concentration found in any blank, consider the result as a nondetect and flag it with a (U).

- When the concentration of that compound is less than the CRQL and less than 5 times the highest concentration found in any blank, report the resuit: as the CRQL with a (U) qualifier.

- When the concentration of the compound is greater than or equal to 5 times the highest concentration found in any blank, consider the result as positive, and no flag is required.

Sample analytes not detected or detected at levels less than CRQL are reported as the CRQL with a (U) flag added.

Laboratory Control Samples-Ensure that each sample is analyzed in a batch in which an LCS has been performed. Any LCS exceeding internal QC limits set by the laboratory for 1 
given sample matrix shall require all data from the associated batch of samples to be closely inspected.

If no analytical problems are found:

- Data analyzed with the out-of-control point shall be discussed.

If problems are found in the analytical data:

- Ensure that samples associated with the batch were re-analyzed and data from the reanalysis reported.

If holding times are exceeded in the re-analysis, both sets of data shall be presented.

If the LCS results are outside internal laboratory limits and if MS results are outside the CLP limits, the data shall be qualified as follows:

- Qualify nondetects for all samples associated with the LC $\boldsymbol{c}$ as unusable (R).

- Qualify positive results for all samples associated with the LCS as estimated (J).

Surrogates-Ensure that all samples are spiked with the surrogate compounds stated in the specific volatile method. Control limits must be established by the laboratory for each surrogate.

If any surrogate recovery is out of specification but greater than $10 \%$ recovery:

- Flag positive results as estimated (J).

- Flag negative results with the CRQL as estimated (UJ).

If any surrogate shows less than $10 \%$ recovery:

- Flag positive results as estimated (J).

- Flag nondetects as unusable (R).

If any blank has surrogates out of specification:

- Flag results using professional judgement.

If zero recovery is reported:

- The reviewer should request the sample chromatograms and examine them to determine if the surrogate may be present but slightly outside its retention time window. If this is the case, in addition to assessing surrogate recovery for quantitative bias, the overriding consideration is to investigate qualitative validity of the analysis. 
If the surrogate is not present:

- Flag positive results as estimated (J).

- Flag all nondetects as unusable (R).

Matrix Spike/Matrix Spike Duplicate-Ensure that an MS/MSD has been associated with each sample. Control limits must be established by the laboratory for each spiking compound. These criteria cannot be used alone to evaluate precision and accuracy. Flagging is not required.

Compound Identification-Ensure that retention times of reported compounds fall within the calculated window for the two chromatographic columns. Second-column confirmation is mandatory.

If the qualitative criteria for the two-column confirmation were not met:

- Qualify all positive results as unusable (R).

\subsubsection{Gas Chromatography/Mass Spectrometer Semivolatile Organics}

Validation for GC/MS semivolatile organics will essentially follow the CLP Functional Validation Guidelines. The criteria below are based on the February 1988 CLP SOW Revision OLM01.8 (EPA 1988e).

Holding Times-Ensure that holding times have been met and samples have been preserved. Both samples and extracts must be preserved at $4^{\circ} \mathrm{C}$. Water samples must be extracted within 7 days, and the extract must be analyzed within 40 days of extraction date. Soil samples must be extracted within 14 days, and the extract must be analyzed within 40 days of extraction date.

If holding time was exceeded or samples were not preserved:

- Document that samples were not properly preserved.

- Document that holding times were exceeded.

- Flag all associated positive results as estimated (J).

- Flag all associated sample quantitation limits as estimated (UJ).

If holding time is grossly exceeded:

- Use best professional judgment as to data reliability. All associated nondetect data may be flagged as unusable (R).

Gas Chromatograph/Mass Spectrometer Tuno-Ensure that a decafluorotriphenylphosphine tune, meeting the CLP criteria, is completed every $12 \mathrm{~h}$ of sample analysis and that each sample is associated with a tune. 
If tunes do not meet the expanded criteria, as listed below:

$\mathbf{m} / \mathbf{z} \quad$ Ion abundance criteria

$51 \quad 30.0$ to $80.0 \%$ of $\mathrm{m} / \mathrm{z} 198$

68 less than $2.0 \%$ of $\mathrm{m} / \mathrm{z} 69$

69 present

70 less than $2.0 \%$ of $\mathrm{m} / \mathrm{z} 69$

$127 \quad 25.0$ to $75 \%$ of $\mathrm{m} / \mathrm{z} 198$

197 less than $1.0 \%$ of $\mathrm{m} / \mathrm{z} 198$

198 base peak, $100 \%$ relative abundance

$199 \quad 5.0$ to $9.0 \%$ of $\mathrm{m} / \mathrm{z} 198$

$275 \quad 10.0$ to $30.0 \%$ of $\mathrm{m} / \mathrm{z} 198$

365 greater than $0.75 \%$ of $\mathrm{m} / \mathrm{z} 198$

441 present, but less than $\mathrm{m} / \mathrm{z} 443$

$442 \quad 40.0$ to $110 \%$ of $\mathrm{m} / \mathrm{z} 198$

$443 \quad 15.0$ to $24.0 \% \mathrm{ofm} / \mathrm{z} 442$

- Flag associated data as unusable (R).

Initial Calibration-The response factor of the compounds listed in Table 7.10 must meet the minimum RRF criteria at each concentration level and maximum \%RSD criteria for the initial calibration with allowances made for up to four compounds. However, the RRFs for those two compounds must be greater than or equal to 0.010 , and the $\%$ RSD of those four compounds must be less than or equal to $40 \%$ for the initial calibration to be acceptable. However, these compounds will still be qualified per appropriate data validation guidelines.

If any compound has an RRF of less than that listed in Tables 7.10 or 7.11:

- Flag positive results for that compound as estimated (J).

- Flag nondetects for that compqund as unusable (R).

If any compound listed in Table 7.10 has a \%RSD of greater than 20.5:

- Flag positive results for that compound as estimated (J).

- Nondetects may be qualified using professional judgment.

Continuing Caibration-If any compound exceeds the minimum RRF of the analytes listed in Tables 7.10 or 7.11:

- Flag positive results for that compound as estimated (J).

- Flag nondetects for that compound as unusable (R). 
Table 7.10 (continued)

Table 7.10 Minimum response factors for initial calibration of semivolatile organics-CLP SOW Revision OLM01.8

\begin{tabular}{lc||lc}
\hline $\begin{array}{l}\text { Semivolatile } \\
\text { compounds }\end{array}$ & $\begin{array}{c}\text { Minimum } \\
\text { RRF }\end{array}$ & $\begin{array}{l}\text { Semivolatile } \\
\text { compounds }\end{array}$ & $\begin{array}{c}\text { Minimum } \\
\text { RRF }\end{array}$ \\
\hline Phenol & 0.800 & Dibenzofuran & 0.800 \\
bis(-2-Chloroethyl)ether & 0.700 & 2,4-Dinitrotoluene & 0.200 \\
2-Chlorophenol & 0.800 & 4-Chlorophenyl-phenylether & 0.400 \\
1,3-Dichlorobenzene & 0.600 & Fluorene & 0.900 \\
1,4-Dichlorobenzene & 0.500 & 4-Bromophenyl-phenylther & 0.100 \\
1,2-Dichlorobenzene & 0.400 & Hexachlorobenzene & 0.100 \\
2-Methylphenol & 0.700 & Pentachlorophenol & 0.050 \\
4-Methylphenol & 0.600 & Phenanthrene & 0.700 \\
N-Nitroso-Di-propylamine & 0.500 & Anthracene & 0.700 \\
Hexachloroethane & 0.300 & Fluoroanthene & 0.600 \\
Nitrobenzene & 0.200 & Pyrene & 0.600 \\
Isophrone & 0.400 & Benzo(a)anthracene & 0.800 \\
2-Nitrophenol & 0.100 & Chrysene & 0.700 \\
2,4-Dimethylphenol & 0.200 & Benzo(b)fluoranthene & 0.700 \\
bis(2-2-Chloroethoxy)methane & 0.300 & Benzo(k)fluoranthene & 0.700 \\
2,4-Dichloropehnol & 0.200 & Benzo(a)pyrene & 0.700 \\
1,2,4-Trichlorobenzene & 0.200 & Indeno(1,2,3-cd)pyrene & 0.500 \\
Naphthalene & 0.700 & Dibenzo(a,h)anthracene & 0.400 \\
4-Chloro-3-methylpheno & 0.200 & Benzo(g,h,i,)perylene & 0.500 \\
2-Methylnaphthalene & 0.400 & Nitrobenzene-d5 & 0.200 \\
2,4,6-Trichlorophenol & 0.200 & 2-Fluorobiphenyl & 0.700 \\
2,4,5-Trichlorophenol & 0.200 & Terphenyl-d14 & 0.500 \\
2-Chloronaphthalene & 0.800 & Phenol-d5 & 0.800 \\
Acenaphthylene & 1.300 & 2-Fluorophenol & 0.600 \\
2,6-Dinitrotoluene & 0.200 & 2-Chloropehnol-d4 & 0.800 \\
Acenaphthene & 0.800 & 1,2-Dichloroethane-d4 & 0.400 \\
\hline & & &
\end{tabular}

The following compounds (see Table 7.11) have no maximum \%RSD, or maximum percent criteria; however, these compounds must meet a minimum RRF criterion of 0.010 .

Table 7.11 Compounds that must meet a minimum RRF criterion of 0.010

\begin{tabular}{l||l}
\hline 2,2'-oxybis(1-Chloropropane) & 4-Nitroaniline \\
4-Chloroaniline & 4,6-Dinitro-2-methylphenol \\
Hexachlorobutadiene & N-Nitrosodiphenylamine \\
Hexachlorocyclopentadiene & Di-n-butylphthalate \\
2-Nitroaniline & Butylbenzylphthalate \\
Dimethylphthalate & 3,3'-Dichlorobenzidine \\
3-Nitroaniline & bis(2-Ethylhexyl)phthalate \\
2,4-Dinitrophenol & 2,4,6-Tribromophenol \\
4-Nitrophenol & Carbazole \\
Diethylphthalate & \\
\hline
\end{tabular}


If any compound has a \%D of greater than $25 \%$ :

- Flag positive results for that compound as estimated (J).

- Nondetects may be qualified using professional judgment.

Blanks-Ensure that all associated blanks were analyzed. taken.

If a compound is found in a blank but not found in the associated sample, no action is

If a compound is found in a blank and the associated sample the following two rules (5x and 10x) apply:

- The $10 x$ Rule applies to those phthalate esters that are common laboratory contaminants.

- When the concentration of that compound is greater than or equal to the CRQL but less than 10 times the highest concentration found in any blank, consider the result as a nondetect and flag it with a (U).

- When the concentration of that compound is less than the CRQL and less than 10 times the highest concentration found in any blank, report the result as the CRQL with a (U) qualifier.

- When the concentration of the compound is greater than or equal to 10 times the highest concentration found in any blank, the result is considered as positive, and no flag is required.

- The 5x Rule applies to all compounds other than the four common laboratory contaminants listed previously.

- When the concentration of that compound is greater than or equal to the CRQL but less than 5 times the highest concentration found in any blank, consider the result as a nondetect and flag it with a (U)

- When the concentration of that compound is less than the CRQL and less than 5 times the highest concentration found in any blank, report the result as the CRQL with a (U) qualifier.

- When the concentration of the compound is greater than or equal to 5 times the highest concentration found in any blank, consider the result as positive, and no flag is required.

Sample analytes not detected or detected at levels less than CRQL are reported as the CRQL with a (U) flag added.

If gross contamination exists:

- Flag all compounds affected as unusable (R).

If inordinate amounts of other compounds and/or TICs are found in any blank:

- Note this in the validation comments. 
Laboratory Control Samples-Ensure that each sample is analyzed in a batch in which an LCS has been performed. Any LCS exceeding internal QC limits set by the laboratory for a given sample matrix shall require all data from the associated batch of samples to be closely inspected.

If no analytical problems are found:

- Data analyzed with the out-of-control point shall be discussed.

If problems are found in the analytical data:

- Ensure that samples associated with the batch were re-analyzed and data from the reanalysis reported.

If holding times are exceeded in the re-analysis, both sets of data shall be presented.

If the LCS results are outside internal laboratory limits and if MS results are outside the CLP limits, the data shall be qualified as follows:

- Qualify nondetects for all samples associated with the LCS as unusable (R).

- Qualify positive results for all samples associated with the LCS as estimated (J).

Surrogates-Ensure that surrogate recoveries are within CLP limits.

If any tr.o surrogate recoveries are outside the limits in any one fraction or any one surrogate in any fraction is below $10 \%$ recovery, there should have been a re-analysis of the sample by the laboratory.

If two or more surrogate recoveries in one fraction are out of specification but greater than $10 \%$ recovery:

- Flag positive results as estimated (J).

- Flag negative results with CRQL as estimated (UJ).

If any surrogate shows less than $10 \%$ recovery:

- Flag positive results as estimated (J).

- Flag negative results as unusable (R).

If any blank has surrogates out of specification:

- Flag results using professional judgement.

Matrix Spike/Matrix Spike Duplicato-Ensure that 1 out of 20 samples has been spiked in duplicate. Recoveries shall meet the CLP criteria.

If recoveries do not meet the criteria, examine the LCS data.

If LCS data and MSs exceed limits: 
- Flag data as unusable (R).

If LCS data from the batch are satisfactory:

- Data are usable, and the low recovery shall be discussed in the final report.

Internal Standard Area Performance-Ensure that IS area counts did not vary by more than a factor of $2(-50 \%$ to $+100 \%)$ from the associated calibration standard.

Retention time of the IS must not vary more than $\pm 30 \mathrm{~s}$ from the associated calibration standard.

If an IS area count is outside $-50 \%$ or $+100 \%$ of the associated calibration standard:

- Flag positive results for compounds quantitated using that IS as estimated (J).

- Flag nondetects for compounds quantitated using that IS with the CRQL as estimated (UJ).

If extremely low area counts are reported or if performance exhibits a major, abrupt drop-off, a severe loss of sensitivity is indicated:

- Qualify nondetects as unusable (R). A discussion must be included in the case narrative describing the problem.

\subsubsection{Pesticides/Polychlorinated Biphenyls}

Holding Times-Ensure that holding times have been met and that samples have been properly preserved. Both samples and extracts must be preserved at $4^{\circ} \mathrm{C}$. Water samples must be extracted within 7 days of collection, and analyzed within $\mathbf{4 0}$ days of the extraction date. Soil samples must be extracted within 14 days of sample collection and analyzed within 40 days of the extraction date.

If holding time was exceeded or samples were not preserved:

- Document that samples were not properly preserved.

- Document that holding times were exceeded.

- Flag all positive results as estimated (J).

- Flag all associated sample quantitation limits as estimated (UJ).

If holding time is grossly exceeded:

- Use best professional judgment as to data reliability. The reviewer may flag all associated nondetect data as unusable (R).

The following guidelines are for CLP SOW Revision OLM01.8 methodology:

Calibration-Ensure that an external calibration procedure is used for quantitation by the laboratory. 
If calibration factors are used for sample quantitation:

- For initial calibration, all \%RSD must be less than or equal to $20 \%$.

- For continuing calibration, all \%D must be less than $15 \%$.

If linear regression is used for sample quantitation:

- Verification of the calibration curve is required, and the correlation coefficient must be greater than or equal to 0.995 .

If the criteria for initial calibration are not met:

- Flag all associated quantitative results as estimated (J).

If the criteria for continuing calibration are not met:

- In the primary analysis, flag all associated quantitative results as estimated (J).

- In the confirmation analysis, use professional judgment as to data reliability.

If proper standards have not been analyzed:

- Use professional judgment as to data reliability.

Blanks-Ensure that all associated blanks were analyzed.

If a compound is found in a blank but not found in the associated sample, no action is taken.

If a compound is found in a blank and the associated sample, the tollowing $5 x$ Rule applies:

- When the concentration of that compound is greater than or equal to the CRQL but less than 5 times the highest concentration found in any blank, consider the result as a nondetect and flag it with a (U).

- When the concentration of that compound is less than the CRQL and less than five times the highest concentration found in any blank, report the result as the CRQL with a (U) qualifier.

- When the concentration of the compound is greater than or equal to five times the highest concentration found in any blank, consider the result as positive, and no flag is required.

- If gross contamination exists (i.e., saturated peaks), all affected compounds in the asociated samples should be qualified as unusable and flagged with an (R) qualifier.

Sample analytes not detected or detected at levels less than CRQL are reported as the CRQL with a (U) flag added. 
Pesticide Cleanup Procedures-Ensure that pesticide cleanup procedures are utilized to remove matrix interferences from sample extracts prior to analysis. The use of the Florisil cartridge cleanup procedure significantly reduces matrix interferences caused by polar compounds. Gel permeation chromatography (GPC) is used to remove high molecular weight contaminants that can interfere with the analysis of target analytes. Pesticide cleanup procedures are checked by spiking the cleanup columns and cartridges and verifying the recovery of pesticides through the cleanup procedure.

- Check the data from the GPC calibration check analyses and the Form IX PEST-2 and recalculate some of the \%Rs to verify that the \%Rs of the pesticides in the matrix spike solution are within $80-110 \%$ and that the Aroclor patterns are similar to those of previous standards. Check to make sure that no transcription errors have occurred.

- If Florisil cartridge check criteria are not met, the raw data should be examined for the presence of polar interferences and professional judgement should be used in qualifying the data. If a laboratory chooses to analyze samples under an unacceptable Florisil cartridge check, the APO should be notified.

- If gel permeation criteria are not met, the raw data should be examined for the presence of high-molecular-weight contaminants and professional judgement should he used in qualifying the data. If a laboratory chooses to analyze samples under unacceptable gel permeation criteria, the APO should be notified.

- If zero recovery was obtained for the pesticide compounds and surrogates during either check, the nondetected target compounds may be suspect and the data may be qualified unusable (R).

Laboratory Control Samples-Ensure that each sample is analyzed in a batch in which an LCS has been performed. Any LCS exceeding internal QC limits set by the laboratory for a given sample matrix shall require all data from the associated batch of samples to be closely inspected.

If no analytical problems are found:

- Data analyzed with the out-of-control point shall be discussed.

If problems are found in the analytical data:

- Ensure that samples associated with the batch were re-analyzed and data from the reanalysis reported.

If holding times are exceeded in the re-analysis, both sets of data shall be presented.

If the LCS recoveries criteria are not met, then the LCS results should be used to qualify sample data for the specific compounds that are included in the LCS solution. If the LCS recovery is out on the high end, detected target compounds may be qualified (J). If the LCS recovery is out on the low end, detected target compounds may be qualified (J) and nondetects may be qualified unusable (R). Professional judgement should be used to qualify data for compounds other than those compounds that are included in the LCS. Professional judgement to qualify non-LCS compounds should take into account the compound class, 
compound recovery efficiency, analytical problems associated with each compound, and comparability in performance of the LCS compound to the non-LCS compound.

If more than half of the compounds in the LCS are not within required advisory recovery criteria, then all of the associated detected target compounds should be qualified $(J)$ and associated nondetected compounds should be qualified unusable.

GC/ECD Instrument Performance Check-Ensure that performance checks on the gas chromatograph with electron capture detector (GC/ECD) system are performed to ensure adequate resolution and instrument sensitivity. These criteria are not sample specific. Conformance is determined using standard materials; therefore, these criteria should be met in all circumstances.

\section{Resolution Check Mocture}

- The resolution check mixture must be analyzed at the beginning of every initial calibration sequence on each GC column and instrument used for analysis. The resolution check mixture contains the following pesticides and surrogates:

$\begin{array}{ll}\text { gamma-Chlordane } & \text { Endrin ketone } \\ \text { Endosulfan I } & \text { Methoxychlor } \\ 4,4^{\prime}-\mathrm{DDE} & \text { Tetrachloro-m-xylene } \\ \text { Dieldrin } & \text { Decachlorobiphenyl }\end{array}$

Endosulfan sulfate

- The depth of the valley between two adjacent peaks in the resolution check mixture, must be greater than or equal to $60.0 \%$ of the height of the shorter peak.

\section{Performance Evaluation Muctures}

- The performance evaluation mixture (PEM) must be analyzed at the beginning (following the resolution check mixture) and at the end of the initial calibration sequence. The PEM must also be analyze at the beginning of every other 12-h analytical period. The PEM contains the following pesticides and surrogates:
gamma-BHC
Endrin
alpha -BHC
Methoxychlor
4,4'-DDT
Tetrachloro-m-xylene
beta-BHC
Decachlorobiphenyl

- The resolution of adjacent peaks for the PEM injections in each calibration (initial and continuing) must be $100 \%$ for both GC columns.

- The absolute retention times of each of the single component pesticides and surrogates in all PEM analyses must be within the specific retention time windows centered around the mean retention times determined from the three-point initial calibration using the Individual Standard Mixtures. 
- The relative percent difference (RPD) between the calculated amount and the true amount for each of the single component pesticides and surrogates in the PEM analyses must be less than or equal to $25.0 \%$.

- The percent breakdown is the amount of decomposition that 4,4'-DDT and Endrin undergo when analyzed on the GC column. For Endrin, the percent breakdown is determined by the presence of Endrin aldehyde and/or Endrin ketone in the GC chromatogram. For 4,4-DDT, the percent breakdown is determined from the presence of $4,4^{\prime}-\mathrm{DDD}$ and/or $4,4^{\prime}-\mathrm{DDE}$ in the GC chromatogram.

- The percent breakdown for both 4,4'-DDT and Endrin in each PEM must be less than or equal to 20.0 for both GC columns.

- The combined percent breakdown for 4,4'- DDT and Endrin in each PEM must be less than or equal to 30.0 for both $\mathrm{GC}$ columns.

\section{Evaluation of the Resolution Check Mixture}

- Verify from the Form VIII PEST (Form VIII LCP) that the resolution check mixture was analyzed at the beginning of the initial calibration sequence on each $G C$ column and instrument used for analysis.

- Check the data and Form VI PEST-1 (Form Vi CLP-4) to verify that the resolution criteria between two adjacent peaks for the required compounds is less than or equal to $60 \%$.

\section{Evaluation of the Performance Evaluation Micture}

- Verify from the Form VIII PEST (Form VIII LCP) that the PEM was analyzed at the proper frequency and position sequence.

- Check the PEM data from the initial and continuing calibrations to verify that the resolution between adjacent peaks is $100 \%$ on both $\mathrm{GC}$ columns.

- Check the PEM data from the initial and continuing calibrations and Form VII PEST-I solvent vent that the absolute retention times for the pesticides in each analysis are within the calculated retention time windows based on the mean RT from the three-point initial calibration.

- Verify that the RPD between the calculated amount and the true amount for each of the pesticides and surrogates is less than or equal to $25.0 \%$.

- Verify that the individual breakdowns for 4,4-DDT and Endrin are less than or equal to $20.0 \%$ and that the combined breakdown is less than or equal to $30.0 \%$.

- Resolution check mixture: If resolution criteria are not met, the quantitative results may not be accurate because of inadequate resolution. Detected target compounds that were not adequately resolved should be qualified with (J). Qualitative identifications may also be questionable if co-elution exists. Nondetects with retention times in the region of co- 
elution may not be valid depending on the extent of the problem. Professional judgement should be used to determine the need to qualify data as unusable (R).

- PEM retention times: Retention time windows are used in qualitative identification. If the retention times of the pesticides in the PEM do not fall within the retention time windows, the associated sample results should be carefully evaluated. All samples injected after the last in-control standard are potentially affected.

- For the affected samples, check to see if the sample chromatograms contain any peaks that are close to the expected retention time window of the pesticide of interest. If no peaks are present either within or close to the retention time window of the deviant target pesticide compound, there is usually no effect on the data (i.e., nondetected values can be considered valid). Sample data that are potentially affected by standards not meeting the retention time windows should be noted in the data review narrative.

- If the affected sample chromatograms contain peaks that may be of concern (i.e., above the CRQL and either close to or within the expected retention time window of the analyte of interest), the reviewer should determine the extent of the effect on the data and may choose to qualify detected target compounds (NJ) and nondetected target compounds (UJ). In some cases, additional effort by the reviewer may be necessary to determine if sample peaks represent the compounds of interest, for example:

- The reviewer can examine the data package for the presence of three or more standards containing the pesticide of interest that were run within a 72-h period during which the sample was analyzed.

- If three or more such standards are present, the mean and standard deviation of the retention time window can be re-evaluated.

- If all standards and matrix spikes fall within the revised window, the valid positive or negative sample results can be determined using this window.

- PEM resolution: If PEM resolution criteria are not met, the quantitative results may not be accurate because of inadequate resolution. Positive sample results for compounds that were not adequately resolved should be qualified with $(J)$. Qualitative identifications may be questionable if co-elution exists. Nondetected target compounds that elute in the region of co-elution may not be valid depending on the extent of the co-elution problem. Professional judgment should be used to qualify data as unusable (R).

- If RFP criteria are not met, qualify all associated positive results generated during the analytical sequence with $(\mathrm{J})$ and the sample quantitation limits for nondetected target compounds with (UJ).

- 4,4'-DDT/Endrin Breakdown:

- If 4,4' -DDT breakdown is greater than $20.0 \%$ : 
- Qualify all positive results for DDT with (J). If DDT was not detected but DDD and DDE are detected, qualify the quantitation limit for DDT as unusable ( $R)$.

- Qualify positive results for DDD and/or DDE as presumf : vely present at an approximated quantity (NJ).

- If Endrin breakdown is greater than 20.0\%:

- Qualify all positive results for Endrin with (J). If Endrin was not detected but Endrin aldehyde and Endrin ketone are detected, qualify the quantitation limit for Endrin as unusable (R).

- Qualify positive results for Endrin ketone as presumptively present at an approximated quantity (NJ).

- If The combined 4,4'-DDT and Endrin breakdown is greater than 30.0\%:

- Qualify all positive results for DDT and Endrin with (J). If Endrin was not detected but Endrin aldehyde and Endrin ketone are detected, qualify the quantitation limit for Endrin as unusable (R). If DDT was not detected but DDD and DDE are detected, qualify the quantitation limit for DDT as unusable ( $R)$.

- Qualify positive results for Endrin ketone as presumptively present at an approximated quantity (NJ).

- Qualify positive results for DDD and/or DDE as presumptively present at an approximated quantity (NJ).

Surrogates-Ensure that all samples are spiked with the surrogate compounds stated in the specific method. Control limits must be established by the laboratory for each surrogate. The advisory limits for recovery of the surrogates TEMX and DBC are $50-150 \%$ for both water and soil.

If low recoveries (i.e., between 10 and $60 \%$ ) are obtained:

- Flag positive results as estimated (J).

- Flag negative results with the CRQL as estimated (UJ).

If high recoveries (i.e., greater than $180 \%$ ) are obtained:

- Qualify associated detected compound data with (J). Nondetected analytes do not require qualification.

If any surrogate shows less than $10 \%$ recovery:

- Flag positive results as estimated (J).

- Flag nondetects as unusable (R). 
If any blank has surrogates out of specification:

- Flag results using professional judgement.

If zero recovery is reported:

- The reviewer should request the sample chromatograms and examine them to determine if the surrogate may be present but slightly outside its retention time window. If this is the case, in addition to assessing surrogate recovery for quantitative bias, the overriding consideration is to investigate qualitative validity of the analysis.

If the surrogate is not present:

- Flag positive results as estimated (J).

- Flag all nondetects as unusable (R).

Matrix Spike/Matrix Spike Duplicate-Ensure that an MS/MSD has been associated with each sample. Control limits must be established by the laboratory for each spiking compound. These criteria cannot be used alone to evaluate precision and accuracy. Flagging is not required.

Compound Identification-Ensure that retention times of reported compounds fall within the calculated window for two chromatographic columns. Second-column confirmation is mandatory.

If the qualitative criteria for both columns were not met, all target compounds that are reported detected should be considered nondetected. The reviewer may need to use the qualifiers that are specific to pesticides. The reviewer should use professional judgement to assign an appropriate quantitation limit using the following guidance:

- If the misidentified peak was sufficiently outside the target pesticide retention time window, the reported values may be a false positive and should be replaced with the sample CRQL value.

- If the misidentified peak poses an interference with potential detection of a target peak, the reported value should be considered and qualified as unusable (R).

If the data reviewer identifies a peak in both GC column analyses that falls within the appropriate retention time windows but was reported as a nondetect, the compound may be a false negative. Professional judgement should be used to decide if the compound should be included. All conclusions made regarding target compound identification should be included in the data review narrative.

If multicomponent target compounds exhibit marginal pattern-matciing quality, professional judgement should be used to establish whether the differences are because of environmental "weathering" (i.e., degradation of the earlier eluting peaks relative to the later eluting peaks). If the presence of a multicomponent pesticide is strongly suggested, results should be qualified as presumptively present $(\mathrm{N})$. 
If an observed pattern closely matches more than one Aroclor, professional judgement should be used to decide whether the neighboring Aroclor is a better match or if multiple Aroclors are present.

Compound Quantitation and Reported Contract-Required Quantitation Limits-Ensure that the reported quantitative results and CRQLs are accurate.

Quantitation limits affected by large, off-scale peaks should be qualified as unusable (R). If the interference is on scale, the reviewer can provide an approximated quantitation limit (UJ) for each effected compound. Note: Single-peak pesticide results are checked for rough agreement between quantitative results obtained on the two GC columns. The potential for co-elution should be considered, and the reviewer should use professional judgment to decide whether a much larger concentration obtained on one column vs the other indicates the presence of an interfering compound. If an interfering compound is indicated, professional judgment must be used to determine how best to report and, if necessary, qualify the data. Contractually, the lower of the two values is reported.

If there are any discrepancies found, the designated representative may contact the laboratory to ot? ain additional information that could resolve any differences. If a discrepancy remains unresolved, the reviewer must determine the best value. Under these circumstances, the reviewer may determine if qualification of the data is warranted. A description of the reasons for data qualification and the qualification applied to the data should be documented in the data review narrative.

The following guidelines are SW-846 methodologies:

Calibration-Ensure that an external calibration procedure is used for quantitation by the laboratory.

If calibration factors are used for sample quantitation:

- For initial calibration, all \%RSD must be less than or equal to 20.

- For continuing calibration, all \%D must be less than 15 .

If linear regression is used for sample quantitation:

- Verification of the calibration curve is required, and the correlation coefficient must be greater than or equal to 0.995 .

In the primary analysis, all standards are analyzed at the beginning of the $72-\mathrm{h}$ period, followed by the proper sample/standard sequence. Confirmation analysis requires a midlevel standard at the beginning of the 72 -h period. The midlevel standard must be repeated after every 10 samples.

If the criteria for initial calibration are not met:

- Flag all associated quantitative results as estimated (J).

If the criteria for continuing calibration are not met: 
- In the primary analysis, flag all associated quantitative results as estimated (J).

- In the confirmation analysis, use professional judgment as to data reliability.

If proper standards have not been analyzed:

- Use professional judgment as to data reliability.

Blanks-Ensure that all associated blanks were analyzed. taken.

If a compound is found in a blank but not found in the associated sample, no action is

If a compound is found in a blank and the associated sample, the following $5 x$ Rule applies:

- When the concentration of that compound is greater than or equal to the CRQL but less than 5 times the highest concentration found in any blank, consider the result as a nondetect and flag it with a (U).

- When the concentration of that compound is less than the CRQL and less than five times the highest concentration found in any blank, report the result as the CRQL with a (U) qualifier.

- When the concentration of the compound is greater than or equal to five times the highest concentraiion found in any blank, consider the result as positive, and no flag is required.

Sample analytes not detected or detected at levels less than CRQL are reported as the CRQL with a (U) flag added.

Laboratory Control Samples-Ensure that each sample is analyzed in a batch in which an LCS has been performed. Any LCS exceeding internal QC limits set by the laboratory for a given sample matrix shall require all data from the associated batch of samples to be closely inspected.

If no analytical problems are found:

- Data analyzed with the out-of-control point shall be discussed.

If problems are found in the analytical data:

- Ensure that samples associated with the batch were re-analyzed and data from the reanalysis reported.

If holding times are exceeded in the re-analysis, both sets of data shall be presented.

If the LCS results are outside internal laboratory limits and if MS results are outside the CLP limits, the data shall be qualified as follows:

- Qualify nondetects for all samples associated with the LCS as unusable (R). 
- Qualify positive results for all samples associated with the LCS as estimated (J).

Surrogates-Ensure that all samples are spiked with the surrogate compounds stated in the specific method. Control limits must be established by the laboratory for each surrogate.

If any surrogate recovery is out of specification but greater than $10 \%$ recovery:

- Flag positive results as estimated (J).

- Flag negative results with the CRQL as estimated (UJ).

If any surrogate shows less than $10 \%$ recovery:

- Flag positive results as estimated (J).

- Flag nondetects as unusable (R).

If any blank has surrogates out of specification:

- Flag results using professional judgement.

If zero recovery is reported:

- The reviewer should request the sample chromatograms and examine them to determine if the surrogate may be present but slightly outside its retention time window. If this is the case, in addition to assessing surrogate recovery for quantitative bias, the overriding consideration is to investigate qualitative validity of the analysis.

If the surrogate is not present:

- Flag positive results as estimated (J).

- Flag all nondetects as unusable (R).

Matrix Spike/Matrix Spike Duplicate-Ensure that an MS/MSD has been associated with each sample. Control limits must be established by the laboratory for each spiking compound. These criteria cannot be used alone to evaluate precision and accuracy. Flagging is not required.

Compound Identification-Ensure that retention times of reported compounds fall within the calculated window for two chromatographic columns. Second-column confirmation is mandatory.

If the qualitative criteria for two-column confirmation were not met:

- Qualify all positive results as unusable (R).

If GC/MS confirmation was required but not performed:

- Document that samples were not confirmed by GC/MS. 
7.3.6 Metals and Cyanide

Validation for metals and cyanide will essentially follow the CLP Function Validation Guidelines.

Holding Times-Ensure that holding times have been met and that samples were properly preserved. Most metal samples must be analyzed within 6 months of sample collection. The exceptions follow:

- Mercury shall be analyzed within 28 days from date of sample collection.

- Cyanide shall be analyzed within 14 days from date of sample collection.

- Hexavalent chromium shall be analyzed within $24 \mathrm{~h}$ from time of sample collection.

All holding times listed above apply to preserved samples.

If holding time was exceeded or samples were not preserved:

- Document that samples were not properly preserved.

- Document that holding times were exceeded.

- Qualify all associated positive results as estimated (J).

- Qualify all associated sample quantitation limits as estimated (UJ).

- Qualify all associated results that are less than the contract-required detection limit (CRDL) as unusable (R).

Initial Calibration Verification-Ensure that instruments were calibrated daily and each time they were set up.

If the minimum number of standards were not used for initial calibration, or if the instrument was not calibrated daily and each time the instrument was set up:

- Qualify data as unusable (R).

If the correlation coefficient was less than 0.995:

c Qualify results greater than or equal to the IDL as estimated (J).

- Qualify results less than the IDL as estimated (UJ).

Continuing Calibration Verification-Ensure that analysis results fall within the control limits of 90 to $110 \%(\% \mathrm{R})$ of the true value for all analytes, except mercury and cyanide. Analysis results for mercury must fall within control limits of 80 to $120 \%$ recovery. Analysis results for cyanide must fall within control limits of 85 to $115 \%$ recovery.

If the initial calibration verification or continuing calibration verification (CCV) \% R falls outside criteria:

- Qualify results greater than or equal to the IDL as estimated (J). 
- Qualify results less than the IDL as estimated (UJ).

Blanks-Ensure that all associated blanks were analyzed. Each blank shall contai:: less than the detection limit for all analytes.

If contaminant concentration in the associated blank is above the IDL and if the lowest analyte concentration is less than 5 times the blank, sample re-analysis should have been performed.

If re-analysis was not performed:

- Qualify results as (U).

Laboratory Control Samples-Ensure that each sample is analyzed in a batch in which an LCS has been performed. Any LCS outside internal QC limits set by the laboratory for a given sample matrix shall require all data from the associated batch of samples to be closely inspected.

If no analytical problems are found:

- Data analyzed with the out-of-control point shall be discussed.

If problems are found in the analytical data:

- Ensure that samples associated with the batch were re-analyzed and data from the reanalysis reported.

If holding times are exceeded in the re-analysis, both sets of data shall be presented.

If the LCS results are outside internal laboratory limits and if MS results are outside the CLP or other appropriate regulatory limits, the data shall be qualified as follows:

- Qualify nondetects for all samples associated with the LCS as unusable (R).

- Qualify positive results for all samples associated with the LCS as estimated (J).

Matrix Spike-Ensure that the spike \%R is within the limits of 75 to $125 \%$.

If spike recovery is greater than $125 \%$ and reported sample results are less than the IDL:

- Data are acceptable.

If spike recovery is greater than $125 \%$ or less than $75 \%$ and sample results are greater than the IDL:

- Qualify data for these samples as estimated (J).

If spike recovery falls within the range of $30 \%$ to less than $75 \%$ and sample results are less than the IDL: 
- Qualify data for these samples as estimated (UJ).

If spike recovery results fall less than $30 \%$ and sample results are less than the IDL:

- Qualify data for these samples as unusable (R).

Duplicato-Ensure that the RPD was within $\pm 20 \%$ (35\% for soil) for sample values greater than or equal to 5 times the CRDL. Also, ensure that a control limit of plus or minus CRDL ( \pm 2 times CRDL for soil) was used for sample values less than 5 times CRDL.

If duplicate analysis results are outside the appropriate control limits:

- Qualify the results for that analyte in all associated samples as esimated (J).

Postdigestion Spike-Ensure that a postdigestion spike was analyzed for FAA analysis. Also, ensure that a postdigestion spike was analyzed for ICP analysis when the MS did not meet criteria (except silver). This data is used to qualify sample results for FAA analysis but not for ICP.

If a postdigestion spike was not analyzed for ICP:

- Document that a postdigestive spike was not analyzed.

If the postdigestion spike recovery for FAA was less than $40 \%$ :

- Qualify results greater than or equal to the IDL as estimated (J).

If the postdigestive spike recovery for FAA is greater than or equal to $10 \%$ but less than 40\%:

- Qualify results less than the IDL as estimated (UJ).

If the postdigestion recovery is less than $10 \%$ :

- Qualify results less than the IDL as unusable (R).

If sample absorbance is less than $50 \%$ of the postdigestion spike absorbance and the postdigestion spike recovery is not within 85 to $115 \%$ :

- Qualify results greater than or equal to the IDL as estimated (J).

- Qualify results less than the IDL as estimated (UJ).

Inductively Coupled Plasma Interference Check Sample-Ensure that an interference check sample interference check sample (ICS) was run at the beginning and end of each sample analysis run (or a minimum of twice per 8-h shift) and that the results were within $\pm 20 \%$ of the true value.

The following applies to samples with concentrations of aluminum, calcium, iron, and magnesium which are comparable to or greater than their respective levels in the ICS. 
If the ICS recovery for an element is greater than $120 \%$ and the sample results are less than the IDL:

- Data is acceptable for use.

If the ICS recovery for an element is greater than $120 \%$ or falls between 50 and $79 \%$ and the sample results are greater than or equal to the IDL:

- Qualify the results for that element in all associated samples as estimated (J).

If the ICS recovery for an element falls between 50 and $79 \%$ and the sample results are less than the IDL:

- Qualify the results for that element in all associated samples as estimated (UJ).

If ICS recovery results for an element fall less than 50\%:

- Qualify the results for that element in all associated samples as unusable (R).

If results greater than the IDL are observed for elements that are not present in the ICS:

- For samples with comparable or higher levels of interferants and with analyte concentrations that approximate those levels found in the ICS, qualify sample results greater than the IDL as estimated (J).

If negative results are observed for elements that are not present in the ICS and their absolute value is greater than the IDL:

- For samples with comparable or higher levels of interferants and with analyte concentrations that approximate those levels found in the ICS, qualify sample results less than the IDL as estimated as estimate (UJ).

If aluminum, calcium, iron, and magnesium are present in the sample at greater than the level in the ICS or other elements are present in the sample at greater than $10 \mathrm{mg} / \mathrm{L}$, investigate the possibility of other interference effects by using Table 7.12. This information should only be considered estimated values. If the estimate is greater than 2 times the CRDL and also greater than $10 \%$ of the reported concentration of the affected element:

- Qualify results as estimated (J).

Method of Standard Addition-Ensure that the Method of Standard Addition (MSA) has been performed when required.

If the MSA was required but not performed:

- Qualify results as estimated (J).

If any samples run by MSA were not spiked at the appropriate levels:

- Qualify results as estimated (J).

If the MSA correlation coefficient is less than 0.995:

- Qualify results as estimated (J). 
Table 7.12. Analyte concentration oquivalent (milligram per liter) arising from interferants at $100 \mathrm{mg} / \mathrm{L}$

\begin{tabular}{|c|c|c|c|c|c|c|c|c|c|c|c|}
\hline \multirow[b]{2}{*}{ Analyte } & \multirow{2}{*}{$\begin{array}{c}\text { Wavelength } \\
\text { nm }\end{array}$} & \multicolumn{10}{|c|}{ Interferant } \\
\hline & & Al & $\mathrm{Ca}$ & $\mathrm{Cr}$ & $\mathrm{Cu}$ & $\mathrm{Fe}$ & $\mathbf{M g}$ & $\mathrm{Mn}$ & $\mathrm{Ni}$ & $\mathrm{Ti}$ & V \\
\hline Aluminum & 308.215 & & & & & & & 0.21 & & & 1.4 \\
\hline Antimony & 206.833 & 0.47 & & 2.9 & & 0.08 & & & & 0.25 & 0.45 \\
\hline Arsenic & 193.696 & 1.3 & & 0.44 & & & & & & & 1.1 \\
\hline Barium & 455.403 & & & & & & & & & & \\
\hline Beryllium & 313.042 & & & & & & & & & 0.04 & 0.05 \\
\hline Boron & 249.773 & 0.04 & & & & 0.32 & & & & & \\
\hline Cadmium & 226.502 & & & & & 0.03 & & & 0.02 & & \\
\hline Calcium & 317.933 & & & 0.08 & & 0.01 & 0.01 & 0.04 & & 0.03 & 0.03 \\
\hline Chromium & 267.716 & & & & & 0.003 & & 0.04 & & & 0.04 \\
\hline Cobalt & 228.616 & & & 0.03 & & 0.005 & & & 0.03 & 0.15 & \\
\hline Copper & 324.754 & & & & & 0.003 & & & & 0.05 & 0.02 \\
\hline Iron & 259.940 & & & & & & & 0.12 & & & \\
\hline Lead & 220.353 & 0.17 & & & & & & & & & \\
\hline Magnesium & 279.079 & & 0.02 & 0.11 & & 0.13 & & 0.25 & & 0.07 & 0.12 \\
\hline Manganese & 257.610 & 0.005 & & 0.01 & & 0.002 & 0.002 & & & & \\
\hline Molybdenum & 202.030 & 0.05 & & & & 0.03 & & & & & \\
\hline Nickel & 231.604 & & & & & & & & & & \\
\hline Selenium & 196.026 & 0.23 & & & & 0.09 & & & & & \\
\hline Silicon & 288.158 & & & 0.07 & & & & & & & 0.01 \\
\hline Sodium & 588.995 & & & & & & & & & 0.08 & \\
\hline Thallium & 190.864 & 0.30 & & & & & & & & & \\
\hline Vanadium & 292.402 & & & 0.05 & & 0.005 & & & & 0.02 & \\
\hline Zinc & 213.856 & & & & 0.14 & & & & 0.29 & & \\
\hline
\end{tabular}




\subsubsection{Wet Chemistry}

Holding Times-Ensure that holding times were met and samples were properly preserved.

If the holding time was exceeded or samples were not preserved:

- Document that samples were not properly preserved.

- Document that holding times were exceeded.

- Flag all associated positive results as estimated (J).

- Flag all associated sample quantitation limits as estimated (UJ).

Initial Calibration-Ensure, at a minimum, that a three-point standard curve bracketing sample concentration is performed daily. The correlation coefficient must meet or exceed 0.995 before the analysis of samples. It is recognized that the correlation coefficient is not an invariable indicator of linearity. However, it is accepted that 0.995 is an achievable value and that linearity is satisfactory when that value is met. If the confidence limits for the individual points are $95 \%$, it follows that the confidence limit for the correlation coefficient is also $95 \%$.

If the minimum number of standards was not used for initial calibration:

- Qualify data as unusable (R).

If the instrument was not calibrated daily before sample analysis:

- Qualify data as unusable (R).

If the correlation coefficient is less than 0.995:

- Qualify sample results greater than or equal to the IDL as estimated (J).

- Qualify sample results less than the IDLs as unusable (R).

Continuing Calibration-Ensure that the continuing calibration falls within the 90 to $110 \%$ control limit.

If the criteria are not met:

- Qualify sample results greater than or equal to the IDL as estimated (J).

- Qualify sample results less than the IDL as unusable (R).

Blank-Ensure that all associated blanks were analyzed.

If the concentration in the sample is less than or equal to the concentration found in the blank:

- The result is considered as a nondetect and flagged as such (U). 
If the concentration in the sample is greater than five times the concentration found in the blank:

- The result is considered positive, and no flag is required.

Laboratory Control Samples-Ensure that each sample is analyzed in a batch in which an LCS has been performed. Any LCS exceeding internal QC limits set by the laboratory for a given sample matrix shall require all data from the associated batch of samples to be closely inspected.

If no analytical problems are found:

- Data analyzed with the out-of-control point shall be discussed.

If problems are found in the analytical data:

- Ensure that samples associated with the batch were re-analyzed and data from re-analysis reported.

If holding times are exceeded ir ine re-analysis, both sets of Jata shall be presented.

If the LCS results are outside internal laboratory limits and if the MS results are outside the laboratory limits, the data shall be qualified as follows:

- Qualify nondetects for all samples associated with the LCS as unusable (R).

- Qualify positive results for all samples associated with the LCS as estimated (J).

Matrix Spike/Matrix Spike Duplicate-Ensure that an MS/MSD has been associated with each sample. Control limits must be established by the laboratory. These criteria cannot be used alone to evaluate precision and accuracy. Flagging is not required.

\subsubsection{Radiochemical Analysis}

Sample Preservation-Ensure that all samples were properly preserved. ER requires radiological samples to be analyzed within 6 months of collection. There is no technical qualification of data for missed holding times, however. Specific QAPPs should clearly identify the logistical time requirements for completion of radiological results.

If samples were not preserved:

- Document that samples were not properly preserved.

- Qualify all associated positive results as estimated (J).

- Quality all associated sample quantitation limits as estimated (J).

- Qualify all associated results that are less than the minimum detectible activity (MDA) as unusable (R). 
Initial Calibration-Ensure that instruments were calibrated and the geometry properly identified. Calibration standard. nust be identified and distributed over the energy range of interest.

If standards are not identified for the initial calibration, or if the instrument was not calibrated:

- Quality the data as unusable (R).

If standards were not distributed over the energy range of interest:

- Qualify results greater than or equal to the MDA as estimated (J).

- Qualify results less than ihe MDA as estimated (UJ).

Continuing Calibration-Ensure that instrument efficiency has remained consistent and is within the control limits ( 90 to $110 \%$ of initial calibration value).

If the continuing calibration falls outside criteria:

- Qualify results greater than or equal to the MDA as estimated (J).

- Quality results less than the MDA as estimated (UJ).

Blanks-Ensure that all associated blanks were analyzed and that background determinations have been performed. Ensure blank and background determinations contain less than the detection limit reported for all analytes.

If contaminant concentration in associated blanks is above the detection limit and, if the lowest analyte concentration is less than 5 times the blank sample, re-analysis of the blank should have been performed.

If re-analysis was not performed:

- Qualify all sample results less than 5 times the blank value as the reporting limit (U).

Laboratory Control Samples-Ensure that each sample is analyzed in a batch that contains an LCS analysis. Any LCS exceeding internal QC limits set by the laboratory for a given matrix and geometry shall require all data from the associated sample batch to be closely inspected.

If no analytical problems are found:

- Data analyzed with the out-of-control LCS shall be specifically referenced in the discussion of results.

If problems are found in the analytical data:

- Ensure samples associated with the batch were re-analyzed and data from the re-analysis is reported. 
If the LCS results are outside internal laboratory limits and if MS results are outside laboratory limits, data shall be qualified as follows:

- Qualify nondetected analytes for all samples associated with the LCS as unusable (R).

- Qualify positive results for all samples associated with the LCS as estimated (J).

Self-Absorption Factors-Ensure that self-absorption factors have been determined for alpha and beta determination. These factors must be determined over the ranis of solids expected in the samples. It is recommended that the solids content not exceed $200 \mathrm{mg}$ total; a more appropriate level may be $100 \mathrm{mg}$ total solids.

If self-absorption factors have not been determined:

- Qualify all associated sample data as unusable (R).

Matrix Spike-Ensure that the spike \% $\mathrm{R}$ is within limits of 75 to $125 \%$. MDA:

If spike recovery is greater than $125 \%$ and reported sample results are less than the

- Data are acceptable.

If spike recovery is greater than $125 \%$ or less than $75 \%$ and sample results are greater than the MDA:

- Qualify data for these samples as estimated (J). MDA:

If spike recovery falls within the range of 30 to $75 \%$ and sample results are less than the

- Qualify data for these samples as estimated (UJ).

If spike recovery results fall less than $30 \%$ and sample results are less than the MDA:

- Qualify wata for these samples as unusable (R).

Duplica-Ensure the RPD was within $\pm 20 \%$ (35\% for soil) for sample values greater than or equal to 5 times the MDA. Also, ensure a control limit of plus or minus the MDA ( \pm 2 times the MDA for soil) was used for duplicate sample comparison values less than 5 times the MDA.

If duplicate analysis results are outside the appropriate control limits:

- Qualify results for that analyte in all associated batch samples as estimated (J). 


\subsection{LEVEL D DATA VALIDATION GUIDEIINES}

At a minimum, data generated from Level D analyses shall be validated per the CLP criteria as outlined in the following documents.

- Laboratory Data Validation Functional Guidelines for Evaluating Organics Analyses, latest edition (EPA 1988c).

- Laboratory Data Validation Functional Guidelines for Evaluating Inorganics Analyses, latest edition (EPA 1988b).

The LCS validation guidelines for Level D will follow Level C guidelines.

For methods not listed in these documents, a similar procedure outlining validation of holding times, initial calibration, continuing calibration, spikes, LCSs, duplicates, and hlank vs sample results will follow the Level $C$ validation, with the additional cross reference to raw data and recalculation confirmation. The prime contractor and the laboratory may also submit a validation procedure for methods not encompassed by the CLP guidelines. This validation procedure must be approved by the APO.

\subsection{LEVEI E DATA VALIDATION GUIDEINNES}

Level E data review and validation guidelines are dependent upon the analyses requested. Review and validation guidelines must be defined in the project WP before the initiation of sampling. At a minimum, criteria for evaluating holding times, initial and continuing calibration, LCSs, and blanks must be defined.

\subsection{DEFWIIION OF DATA QUALIFIERS}

The terms flag and qualifier are used interchangeably by data validators. The following qualifiers are used to assess the usability of the data in the EPA CLP program and are used for Levels $C$ and D:

Organic

U Indicates compound was analyzed for but not detected. The sample quantitation limit must be corrected for dilution and for percent moisture.

J Indicates an estimated value. This flag is used either when estimating a concentration for TICs where a 1:1 response is assumed, or when the mass spectral data indicate the presence of a compound that meets the identification criteria but the result is less than the sample quantitation limit but greater than zero.

C This flag applies to pesticide results where the identification has been confirmed by GC/MS. Single component pesticides $210 \mathrm{ng} / \mu \mathrm{L}$ in the final extract shall be confirmed by GC/MS. 
B This flag is used when the analyte is found in the associated blank, as well as in the sample. It indicates possible/probable blank contamination and warns the data user to take appropriate action. This flag must be used for a TIC, as well as for a positively identified target compound list (TCL) compound.

E This flag identifies compounds whose concentrations exceed the calibration range of the GC/MS instrument for that specific analysis. This flag will not apply to pesticides/PCBs analyzed by GC/electron capture methods. If one or more compounds have a response greater than full scale, the sample or extract must be diluted and re-analyzed. If the dilution of the extract causes any compounds identified in the first analysis to be below the calibration range in the second analysis, the results of both analyses shall be reported.

D This flag identifies all compounds identified in an analysis at a secondary dilution factor. If a sample or extract is re-analyzed at a higher dilution factor, as in the " $E$ " flag above, the "DL" suffix is appended to the sample number on the Form I for the diluted sample, and all concentration values reported on that Form I are flagged with the "D" flag.

A This flag indicates that a TIC is a suspected aldol-condensation product.

$\mathrm{X}$ Other specific flags and footnotes may be required to properly define the results. If used, they must be fully described and such description attached to the Sample Data Summary Package and the Case Nerrative. If more than one is required, use " $Y$ " and " $Z$," as needed. If more than five qualifiers are required for a sample result, use the " $X$ " flag to combine several flags, as needed. For instance, the " $X$ " flag might combine the " $A$," "B," and " $D$ " flags for some sample.

$\mathbf{R} \quad \mathrm{QC}$ indicates that data are not usable (compound may or may not be present). Resampling and re-analysis are necessary for verification.

Q No analytical result.

Inorganic

E The reported value is estimatid because of the presence of interference. An explanatory note must be included under "Comments" on the cover page (if the problem applies to all samples) or on the specific FORM I-IN (if it is an isolated problem).

M Duplicate injection precision not met.

N Spiked sample recovery not within control limits.

S The reported value was determined by the MSA.

W Postdigestion spike for FAA analysis is out of control limits (85 to 115\%); sample absorbance is less than $50 \%$ of spike absorbance.

- Duplicate analysis not within control limits.

$+\quad$ Correlation coefficient for the MSA is less than 0.995 . 
M (Method) Qualifier

"P" for ICP

"A" for Flame Atomic Absorption

" $F$ " for Furnace Atomic Absorption

"CV" for Manual Cold Vapor Atomic Absorption

"AV" for Automated Cold Vapor Atomic Absorption

"AS" for Semiautomated Spectrophotometric

"C" for Manual Spectrophotometric

"T" for Titrimetric

"NR" if the analyte is not required to be analyzed 


\section{REFERENCES}

American National Standards Institute, 1991. Measurement Quality Assurance for Radioassay Laboratories, ANSI N42.2, Revised January 5.

Cromartie, D. W., et. al., 1975. "Residues of Organochlorine Pesticides and Polychlorinated Biphenyls: Autopsy Data for Bald Eagles." J. Pestic. Monit. 9:11-14.

Energy Systems (Martin Marietta Energy Systems, Inc.), 1991. Environmental Restoration Division Quality Assurance Program Plan, ES/ER/TM-4/R1, Environmental Restoration Division, September.

EPA (U. S. Environmental Protection Agency), 1979. Emission Assessments of Conventional Stationary Combustion Systems: Methods and Procedures Manual for Sampling and Analysis, Interagency Energy/Environmental R\&D Program, Industrial Environmental Laboratory Research Laboratory, RTP. EPA-600/7-79-029a.

EPA (U. S. Environmental Protection Agency), 1983. Interim Guidelines and Specifications for Preparing Quality Assurance Project Plans, QAMS 005/80, EPA-600/4-83-004, February.

EPA (U. S. Environmental Protection Agency), 1984. Eastern Environmental Radiation Facility Radiochemistry Procedures Manual. Office of Radiation Programs, EPA 520/ 5-84-006.

EPA (U. S. Environmental Protection Agency), 1986. Test Methods for Evaluating Solid Waste, Physical/Chemical Methods, SW-846, 3rd ed., November 29.

EPA (U. S. Environmental Protection Agency), 1987. Data Quality Objectives for Remedial Response Activities Development Process, EPA Office of Solid Waste and Emergency Response (OSWER) Directive 9355.0-7B, March.

EPA (U. S. Environmental Protection Agency), 1988a. Guidance for Conducting Remedial Investigations and Feasibility Studies Under CERCLA, Interim Final, EPA Office of Solid Waste and Emergency Response (OSWER) Directive 9355.3-01, October.

EPA (U. S. Environmental Protection Agency), 1988b. Laboratory Data Validation Functional Guidelines for Evaluating Inorganics Analyses, Hazardous Site Control Division, July 1.

EPA (U. S. Environmental Protection Agency), 1988c. Laboratory Data Validation Functional Guidelines for Evaluating Onganics Analyses, Hazardous Site Control Division, February 1.

EPA (U. S. Environmental Protection Agency), 1988d. USEPA Contract Laboratory Program Statement of Work for Inorganics Analysis, ILM01.0 and ILM02.0 plus revision 1, July.

EPA (U. S. Environmental Protection Agency), 1988e. USEPA Contract Laboratory Program Statement of Work for Organics Analysis, OLM01.0 plus revisions OLM01.1-.8, February. 
EPA (U. S. Environmental Protection Agency), 1989. RCRA Facility Investigation Guidance, EPA 530/SW-89-031.

NIOSH (National Institute of Occupational Safety and Health), 1984. NIOSH Manual of Analytical Methods, Publication No. 84-100, U.S. Department of Health and Human Services, Cincinnati, Ohio, February.

International Standard Organization (ISO) for Standardization, 1987. Quality Management and Quality Assurance Standards-Guidelines for Selection and Use, first edition, Standard 9000, European Economic Community, Geneve, Switzerland. 


\section{BIBLIOGRAPHY}

American National Standards Institute/American Society of Mechanical Engineers (ANSI/ASME), Quality Assurance Program Requirements for Nuclear Facilities, NQA-1, 1986 ed., July 1, 1986.

American Society for Testing and Materials (ASTM), Standard Specification for Reagent Water, Standard D 1193 - 77 (reapproved 1983).

Energy Systems (Martin Marietta Energy Systems, Inc.), Environmental Surveillance Procedures Quality Control Program, Revision 1, ESH/Sub/87-21706/1, January 31, 1990.

EPA (U. S. Environmental Protection Agency), Guidance for Data Useability in Risk Assessment, Solid Waste and Emergency Response, EPA/540/G-90/008, October 1990.

EPA (U. S. Environmental Protection Agency), Quality Assurance Glossary and Acronyms, Office of Research and Development, February 8, 1991.

F. M. Garfield, "Quality Assurance Principles for Analytical Laboratories," Association of Official Analytical Chemists, Arlington, VA, 1984 ed.

Hazardous Waste Remedial Actions Program, Martin Marietta Energy Systems, Inc., HAZWRAP SCO Implementation Plan, Quality Assurance Requirements, DOE/HWP-38, November 1987.

Hazardous Waste Remedial Actions Program, Martin Marietta Energy Systems, Inc., Quality Control Requirements for Field Methods, DOE/HWP-69/R1, July 1990.

Hazardous Waste Remedial Actions Program, Martin Marietta Energy Systems, Inc., Requirements for Quality Control of Analytical Data, DOE/HWP-65/R1, July 1990.

Martin Marietta Energy Systems, Inc., Environmental Surveillance Procedures Quality Control Program, ESH/Sub/87-21706/1.

U.S. Department of Energy, Office of Environmental Audit, The Environmental Survey Manual, Appendix D-Part 4, 2nd ed., January 1989.

U.S. Environmental Protection Agency, Office of Radiation Programs, Eastern Environmental Radiation Facility Radiochemistry Procedures Manual, August 1984.

U.S. Environmental Protection Agency, "Hazardous Waste Operations and Emergency Response," Federal Register, 29 CFR 1910.120, Vol. 54, No. 2, March 6, 1989.

U.S. Environmental Protection Agency, Methods for Chemical Analysis of Water and Wastes, EPA-600/4-79-020, revised March 1983.

U.S. Environmental Protection Agency, "Toxic Substances Control; Good Laboratory Practice Standards; Final Rule," Federal Register, Vol. 48, November 29, 1983.

U.S. Food and Drug Administration, "Nonclinical Laboratory Studies; Good Laboratory Practice Regulations," Federal Register, Vol. 43, December 22, 1978. 


\section{GLOSSARY}

Accuracy-the nearness of a result or the mean of a set of results to the true or accepted value. Accuracy is measured by the percent recovery for spikes.

Analyto-a chemical component of a sample to be determined or measured.

Analytical method-defines the sample preparation and instrumentation procedures or steps that must be performed to estimate the quantity of analyte in a sample.

Analytical spike-The addition of a known amount of analyte or compound to the sample prior to analysis to assess the precision of the analytical method.

Appendix IX-key list of analytes for waste analysis. " $\mathrm{t}$ is derived from the Appendix VII list, the "Michigan List," and the priority pollutant list, and is intended to include those compounds accessible by validated methods.

Appendix VII-primary analyte list for RCRA.

Applicable or Relevant and Appropriate Requirements-requirements or standards that are applicable or relevant and appropriate to the hazardous substances or particular circumstances at a site.

Background correction-a technique to compensate for variable background contribution to the instrument signal and the determination of trace metals.

Batch-the number of samples of the same composition that can be prepared at one time. The batch should not exceed 20 samples.

Calibration-the establishment of an analytical correlation between known concentrations of analyte and the instrument response based on the absorbance, emission intensity, peak height, area, or count. Calibration standards must be prepared using the same type of reagents and concentration as used for sample preparation.

Calibration blank-a volume of acidified deionized/distilled water.

Chain of custody-supervisory possession of samples consistent with rules of evidence for submission to legal proceedings. This involves documented transfer of the sample from time of collection through disposal.

Comparability-a qualitative parameter expressing the confidence with which one data set can be compared with another. Sample data should be comparable with other measurement data for similar samples and sample conditions.

Completeness-defined as the percentage of measurements made which are judged to be valid measurements. The completeness goal is to generate sufficient amount of valid data based on project needs. 
Contaminant-any physical, chemical, biological, or radiological substance that has an adverse affect on air, soil, or water.

Continuing calibration-analytical standard run in a specified sequence or time interval to verify that the calibration of the analytical system is in control.

Control limits-a range within which specified measurement results must fall to be compliant. Control limits may be mandatory, requiring corrective action if exceeded, or advisory, requiring that noncompliant data be flagged.

Correlation coefficient-a number that indicates the degree of dependence between two variables (concentration - absorbance). The more dependent they are, the closer the value to 1 . Determined on the basis of the least square fit.

Corrosivity-one of the major evaluation tests to determine if a waste is hazardous. It employs extreme $\mathrm{pH}$ and steel corrosion as criteria for a positive response.

Data quality objectives-qualitative and quantitative statements that specify the quality of the data required to support decision during remedial response activities. Data quality objectives are determined based on the end uses of the data to be collected.

Detection limit-the minimum concentrations that must be accurately and precisely measured by the laboratory and/or specified in the quality assurance plan.

Dissolved metals-analyte elements that have not been digested prior to analysis and that will pass through a $0.45-\mu \mathrm{m}$ filter.

Duplicates-identical splits of individual samples which are analyzed by the laboratory to test for method reproducibility. In this case, samples are split in the laboratory.

Equipment rinsates-the final analyte-free water rinse from equipment cleaning collected daily during a sampling event.

Event-the time the sampling personnel arrive at the site until personnel leave the site for more than $24 \mathrm{~h}$.

Field duplicates-Independent samples collected as closely as possible to the same point in space and time and intended to be identical. Because of the possible loss of volatile analytes, it is necessary to collect collocated samples instead of splits for soil samples that will be analyzed for VOCs. Field duplicates for water samples are collected by filling two or more containers with the same sample. Field duplicates may be used to evaluate the precision of the sampling process or the heterogeneity of the matrix.

Field splits-Portions taken from an original single sample in such a way that their properties are assumed to be identical. Soil splits are homogenized before being split; because homogenization is not acceptable for soil samples submitted for VOC analyses, duplicate (collocated) soil samples are taken instead of splits for such analyses. Split samples are often sent to separate laboratories to help in ascertaining interlaboratory variability or possibly other variability such as in shipping conditions. 
Halogenated organic compound-an organic compound with a halogen (bromine, chlorine, fluorine, iodine) substituted for a hydrogen. Also called halocarbons.

Ignitability-one of the major characteristic tests used to determine if a waste is hazardous.

Instrument detection limit-defined in several ways. For example: (1) that concentration of analyte which produces an output signal twice the root mean square of the background noise may be determined under ideal conditions, or (2) determined by multiplying by 3 the standard deviation obtained for the analysis of a standard solution (each analyte in reagent water) at a concentration of 3 to 5 times instrument detection limit on three nonconsecutive days within 7 consecutive measurements per day.

Internal standards-compounds added to every standard, blank, MS, MSD, sample (for volatile), sample extract (for semivolatile) at a known concentration prior to analysis. Internal standards are used as the basis for quantitation of the target compounds.

Laboratory control sample-a control sample of known composition. Aqueous and solid laboratory control samples are analyzed using the same sample preparation, reagents, and analytical methods employed for samples received.

Laboratory quality assurance coordinator-an employee of a laboratory with no analysis or production responsibilities and who implements QA and $\mathrm{QC}$. This person is responsible for ensuring all quality problems are resolved.

Matrix-The predominant material comprising the sample to be analyzed. The most common matrixes are water, soil/sediment, and sludge.

Matrix spike-an aliquot of a matrix (water or soil) spiked with known quantities of compounds and subjected to the entire analytical procedure to indicate the appropriateness of the method for the matrix by measuring recovery.

Matrix spike duplicate-a second aliquot of the same matrix as the MS that is spiked to determine the precision of the method.

Method blank-an analytical control consisting of all reagents, internal standards, and surrogates which is carried through the entire analytical procedure. It is used to define the level of laboratory background contamination.

Method blankspike-the distilled and/or deionized water, clean soil, or sand spiked with known compounds or elements. The method blank, as defined by the CLP for organics, and the laboratory control sample, as defined by the CLP, may be used as the method blank/spike in the ER Program.

Method detection limits-minimum concentration of a substance that can be measured and reported with $99 \%$ confidence that the value is above zero. The sample is carried through the entire method under ideal conditions. 
Method of standard additions-the addition of three increments of a standard solution (spikes) to sample aliquots of the same size. Measurements are made on the original and after each addition. The slope, $x$-intercept, and $y$-intercept are determined by least-squares analysis. The analyte concentration is determined by the absolute value of the $x$-intercept. Ideally, the spike volume is low relative to the sample volume ( $-10 \%$ of the volume). Standard addition may counteract matrix effects; it will not counteract spectral effects. It is also referred to as standard addition.

Mixed waste-any chemical waste that is also contaminated by radiation.

Out of control-one or more of several conditions indicating unacceptable results.

Percent moisture-the proportion of water in a soil sample determined by drying an aliquot of the sample.

Polynuclear aromatic-a compound consisting of two or more cyclic structures joined together.

Precision-measure of the reproducibility of a set of replicate results among themselves or the agreement among repeat observations made under the same conditions. Precision is measured by the RPD between an MS and MSD or duplicate.

Preparation blank (reagent blank, method blank) -an analytical control that contains distilled, deionized water and reagents which is carried through the entire analytical procedure (digested and analyzed). An aqueous method blank is treated with the same reagents as a sample with a water matrix; a solid method blank is treated with the same reagents as a soil sample.

Purge and trap-an analytical technique used to isolate volatile (purgeable) organics by stripping the compounds from water or soil by a stream or inert gas, trapping the compounds on a porous polymer trap and thermally desorbing the trapped compounds. onto the gas chromatographic column.

Qualitative analysis-any analysis that identifies an analyte or class of analytes but does not provide exact information on the concentration of these target analytes.

Quality assurance-a planned system of activities (program) with the purpose of providing assurance of the reliability and defensibility of data.

Quality control-a routine application of procedures for controlling the monitoring process. $\mathrm{QC}$ is the responsibility of all those performing the hands-on operations in the field and laboratory.

Quantitative analysis-any analysis that provides information as to the specific concentration of anaijtes.

Reagent water-water in which an analyte is not observed at or above the minimum quantitation limit of the parameters of interest. 
Recovery-The numerical ratio of the amount of analyte measured by the laboratory method divided by the known amount of analyte added to the matrix (i.e., spiked sample to be analyzed). Usually expressed as a percentage.

Reporting detection limits-the same as method detection limits, with consideration given for practical limitations such as sample size, matrix interferences, and dilutions.

Representativeness-expressis the degree to which sample data accurately and precisely represent a characteristic of a population, parameter variations at a sampling point, or an environmental condition. Representativeness is a qualitative parameter which is most concerned with the proper design of the sampling program.

Sampling holding times-times used to ascertain the validity of results based on the holding time of the sample from date of collection to date of analysis or sample preparation. Holding times may very depending on the analysis, EPA regional preference, etc.

Semiquantitative analysis-any analysis that provides limited information on the concentration of analytes.

Semivolatile compounds-compounds amenable to extraction with an organic solvent. Used synonymously with base neutral acid or extractable compounds.

Serial dilution-the dilution of a sample by a known factor. When corrected by the dilution factor, the diluted sample must agree with the original undiluted sample within specified limits. Serial dilution may reflect the influence of interferants.

Source water blanks-collected and analyzed to determine the level of contamination introduced into the sample because of sampling technique. They may consist of the source water used in decontamination and steam cleaning. At a minimum, one sample from each event and each source of water must be collected and analyzed.

Spikes-known amounts of specific chemical constituents added by the laboratory to selected samples to test the appropriateness and recovery efficiencies of specific analytical methods within the actual sample matrixes.

Standard deviation-the square root of the variance of a set of values.

Surrogates-compounds added to every blank, sample, MS, MSD, and standard which are used to evaluate analytical efficiency of the method by measuring recovery. Surrogates are brominated, fluorinated, or isotopically labeled compounds not expected to be detected in environmental media. These are used typically in organic methods.

Tentatively identified compounds-compounds detected in samples that are not target compounds, internal standards, or surrogate standards. Up to $\mathbf{3 0}$ peaks (those greater than $10 \%$ of peak areas or heights of nearest internal standards) are subjected to mass spectral library searches for tentative identification.

Variance-some of the squares of the difference between the individual values of a set and the arithmetic mean of the set, divided by one less than the number of values. 
Volatile compounds-compounds amenable to analysis by the purge and trap techniques. Used synonymously with purgeable compounds.

Wet chemistry-a term that is used in reference to the analysis of parameters that are not considered organic or metal (e.g., sulfate).

Additional definitions may be found in the following references:

- American National Standards Institute, Measurement Quality Assurance for Radioassay Laboratories, ANSI N42.2, Revised January 5, 1991.

- U.S. Environmental Protection Agency, Office of Research and Development, Quality Assurance Glossary and Acronyms, February 8, 1991.

- U.S. Environmental Protection Agency, Solid Waste and Emergency Response, Guidance for Data Useability in Risk Assessment, EPA/540/G-90/008, October 1990.

- U.S. Environmental Protection Agency, Test Methods for Evaluating Solid Waste, Physical/Chemical Methods, SW-846 (Draft November 1990). 


\section{INTERNAL DISTRIBUTION}

1. S. M. Adams

2. J. M. Asher

3. L. V. Asplund

4. J. W. Autry

5. M. B. Baer

6 L. D. Bates

7. D. E. Beck

8. C. W. Beeks

9. D. T. Bell

10. D. F. Bennett

11-12. H. D. Bewley

13. S. C. Bieneik

14. B. G. Blaylock

15. W. R. Brown, Jr.

16. V. J. Brumback

17. S. N. Burman

18. I. C. Campbell

19. Charles Clark

20. L. B. Cobb

21-25. J. S. Colley

27. K. W. Cook

28. R. B. Cook

29. E T. Collins

30. D. G. Cope

31. T. Cothran

32. D. Davenport

33. N. W. Duriee

34. J. A. Early

35. T. O. Early

36. J. T. Etheridge

37. C. A Farnsworth

38. R. E Fernstermaker

39. C. J. Ford

40. H. R. Gaddis

41. S. B. Garland

42. P. L. Goddard

43. C. D. Goins

44. J. L Greene

45. J. T. Grumski

46. P. J. Halsey

47. T. L. Hatmaker

48. J. L. Haymore

49. G. J. Hlaynes

50. N. A. Hefty

51. M. M. Heiskell

52. R. M. Hill, Jr.
53. J. A. Hodgins

54. F. O. Hoffman

55. S. K. Holladay

56. R. K. Holmes

57. L. A. Hook

58. C. G. Hudson

59. D. D. Huff

60. L. P. Hull

61. L. D. Hyde

62. G. James

63. L. L. Kaiser

64. K. W. Keever

65. C. W. Kimbrough

66. B. L. Kimmel

67. A. F. Kiriluk

68. T. M. Koepp

69. A. J. Kuhaida

70. J. A Lea

71. S. M. Leone

72. N. A. Luedike

73. L. E. May

74. A. F. McClanahan

75. G. D. Mencer

76. D. G. Miller

77. J. Q. Miller

78. N. J. Montgumery

79. K. M. Nelson

80. H. C. Newsom

81. T. J. Newsom

82. J. D. Noblit

83. M. J. Norris

84-85. P. T. Owen

86. G. J. Pardue

87. J. G. Parrott

88. J. S. Phillips

89. G. A. Plante

90. G. M. Powers

91. P. N. Rader

92. L. O. Ramseth, Paducah

93. T. G. Ramsey

94. J. Reatsnyder

95. W. D. Rideout

96. G. E. Rymer

97. P. A. Schrandt

98. B. J. Scott

99. D. E Searle

100. L. G. Shipe

101. J. K. Siberell 
102. R. H. Snyder

103. M. L. Sollenberger

104. J. V. Spence

105. R. J. Spence

106. W. J. Spetnagel

107. A. M. Stanley

108. T. D. Taylor

109. W. W. Thompson, Jr.

110. T. S. Tison

111. K. S. Turner

112. G. L. Underberg

113. J. H. Vanderlan

114. S. D. Van Hoesen

115. C. J. Vanmeter

116. L D. Voorhees

117. B. D. Walker

118. C. S. Walker
119. K. L. Walker

120. A. E. Walzer

121. J. S. Watson

122. D. R. Watkins

123. S. C. Wells

124. D. C. White

125. R. K. White

126. L. M. Whitehead

127. A. S. Will

128. P. A Williams

129. M. A. Woody

130. Central Research Library

131. K-25 Technical Library

132. Y-12 Technical Library

133-137. ER Document Management Center-RC

138. Y-12 Document Center

139. ORNL Laboratory Records

\section{EXTERNAL DISTRIBUTION}

140. G. W. Bodenstein, DOE Oak Ridge Field Office, P.O. Box 2001, Oak Ridge, TN 37831-8541

141. V. F. Boston, DOE Oak Ridge Field Office, P.O. Box 2901, Oak Ridge, TN 37831-8541.

142. D. M Carden, DOE Oak Ridge Field Office, P.O. Box 2001, Oak Ridge, TN 37831-8541

143. Division Office of Quality Assurance, DOE Oak Ridge Field Office, P. O. Box 2001, Oak Ridge, TN 37831-8541

144. D. W. Dollins, DOE Oak Ridge Field Office, Paducah, Kentucky, Building C100, DOE Office

145. R. C. Edwards, DOE Oak Ridge Field Office, Paducah, Kentucky, Building C100, DOE Office

146. C. S. Gist, DOE Oak Ridge Field Office, P.O. Box 2001, Oak Ridge, TN 37831-8541

147. S. L Lankford, DOE Oak Ridge Field Office, P.O. Box 2001, Oak Ridge, TN 37831-8541

148. T. A. Larkin, DOE Oak Ridge Field Office, P.O. Box 2001, Oak Ridge, TN 37831-8541

149. R. W. Meehan, Department of Energy Portsmouth Site Office

150-151. R. L. Nace, Branch Chief, Nonenrichment Facilities, Oak Ridge Program Division, Office of Eastern Area Programs, Office of Environmental Restoration, EM-423, Trevion 2, U.S. Department of Energy, Washington, DC 20585

152. D. G. Page, DOE Oak Ridge Field Office, P.O. Box 2001, Oak Ridge, TN 37831-8541

153. S. P. Riddle, DOE Oak Ridge Field Office, P.O. Box 2001, Oak Ridge, TN 37831-8541

154. J. D. Rothrock, DOE Oak Ridge Field Office, P.O. Box 2001, Oak Ridge, TN 37831-8541

155-164. R. C. Sleeman, DOE Oak Ridge Field Office, P.O. Box 2001, Oak Ridge, TN 37831-8541

165. R. R. Stone, Radian Corporation, 120 S. Jefferson Circle, Oak Ridge, TN 37830

166. J. T. Sweeney, DOE Oak Ridge Field Office, P.O. Box 2001, Oak Ridge, TN 37831-8541

167. D. W. Swindle, Radian Corporation, 120 S. Jefferson Circle, Oak Ridge, TN 37830

168-169. H. M. Thron, Chief, Enrichment Facilities, Oak Ridge Program Division, Office of Eastern Area Programs, Office of Environmental Restoration, EM-423, Trevion 2, U.S. Department of Energy, Washington, DC 20585

170. M. A. Travaglini, DOE Oak Ridge Field Office, P.O. Box 2001, Oak Ridge, TN 37831-8541

171. T. J. Wheeler, Department of Energy, Oak Ridge Operations, P.O. Box 2001, Oak Ridge, TN 37831-8540

172-173. Office of Scientific and Technical Information, P.O. Bo: 62, Oak Ridge, TN 37831 

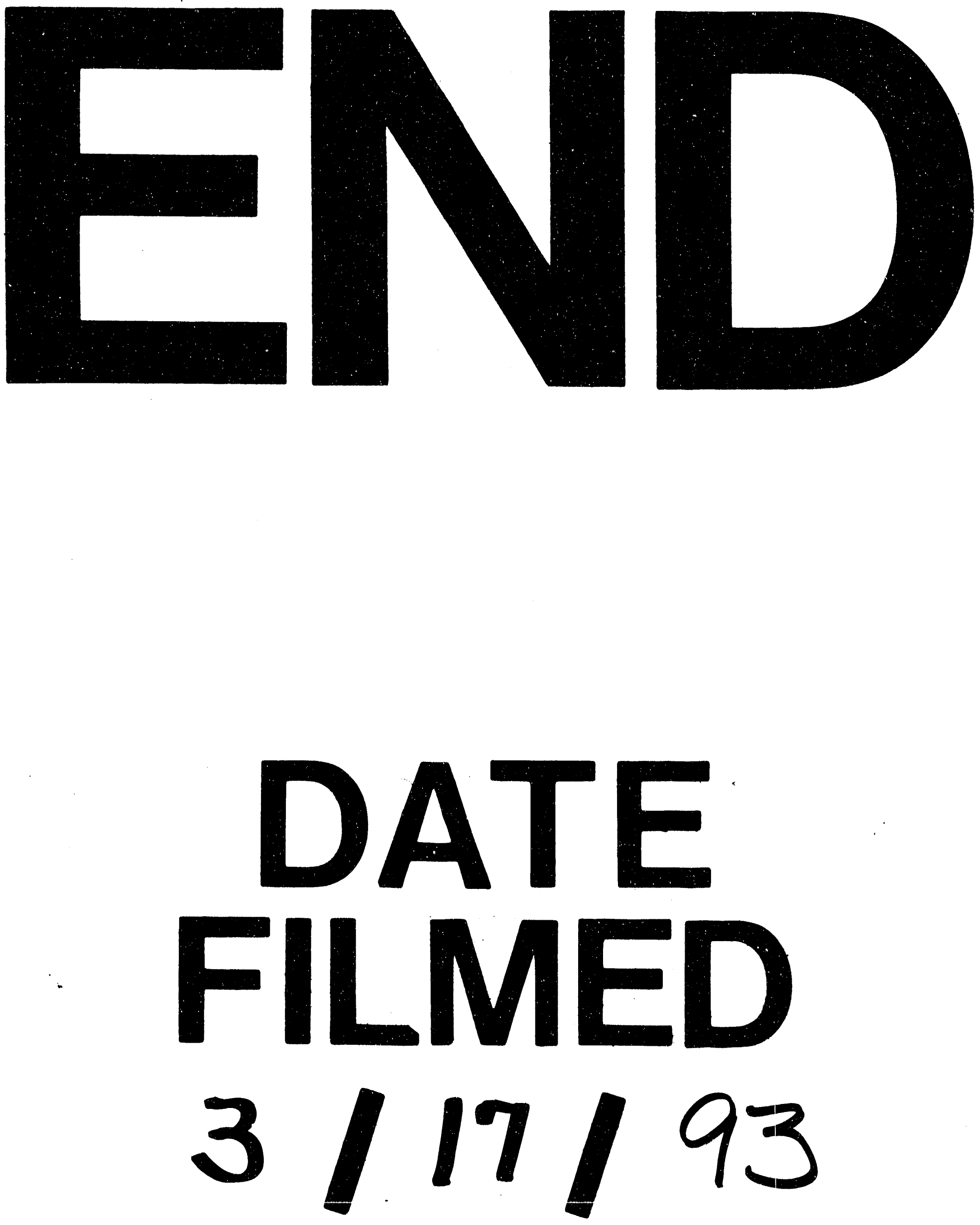

1 
